\title{
SEMICANONICAL BASES AND PREPROJECTIVE ALGEBRAS
}

\author{
BY CHRISTOF GEISS, BERNARD LECLERC AND JAN SCHRÖER
}

Dedicated to Claus Michael Ringel on the occasion of his sixtieth birthday

\begin{abstract}
We study the multiplicative properties of the dual of Lusztig's semicanonical basis. The elements of this basis are naturally indexed by the irreducible components of Lusztig's nilpotent varieties, which can be interpreted as varieties of modules over preprojective algebras. We prove that the product of two dual semicanonical basis vectors $\rho_{Z^{\prime}}$ and $\rho_{Z^{\prime \prime}}$ is again a dual semicanonical basis vector provided the closure of the direct sum of the corresponding two irreducible components $Z^{\prime}$ and $Z^{\prime \prime}$ is again an irreducible component. It follows that the semicanonical basis and the canonical basis coincide if and only if we are in Dynkin type $\mathbb{A}_{n}$ with $n \leqslant 4$. Finally, we provide a detailed study of the varieties of modules over the preprojective algebra of type $\mathbb{A}_{5}$. We show that in this case the multiplicative properties of the dual semicanonical basis are controlled by the Ringel form of a certain tubular algebra of type $(6,3,2)$ and by the corresponding elliptic root system of type $\mathbb{E}_{8}^{(1,1)}$.
\end{abstract}

(C) 2005 Elsevier SAS

RÉSUMÉ. - Nous étudions les propriétés multiplicatives de la base duale de la base semi-canonique de Lusztig. Les éléments de cette base sont naturellement paramétrés par les composantes irréductibles des variétés nilpotentes de Lusztig, qui peuvent être interprétées comme variétés de modules sur les algèbres préprojectives. Nous démontrons que le produit de deux vecteurs $\rho_{Z^{\prime}}$ et $\rho_{Z^{\prime \prime}}$ de la base semicanonique duale est encore un vecteur de la base semi-canonique duale si la somme directe des composantes irréductibles $Z^{\prime}$ et $Z^{\prime \prime}$ est encore une composante irréductible. Il en résulte que les bases canonique et semi-canonique ne coïncident que pour le type de Dynkin $\mathbb{A}_{n}$ avec $n \leqslant 4$. Finalement, nous étudions en détail les variétés de modules sur l'algèbre préprojective de type $\mathbb{A}_{5}$. Nous montrons que dans ce cas les propriétés multiplicatives de la base semi-canonique duale sont contôlées par la forme de Ringel d'une algèbre tubulaire de type $(6,3,2)$ et par le système de racines elliptique de type $\mathbb{E}_{8}^{(1,1)}$ qui lui est associé.

(c) 2005 Elsevier SAS

\section{Contents}

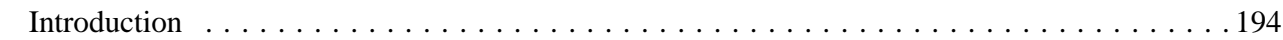

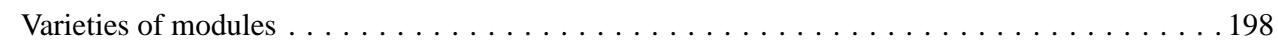

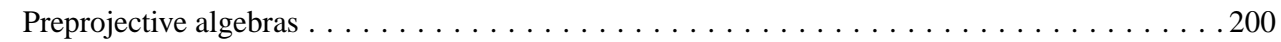

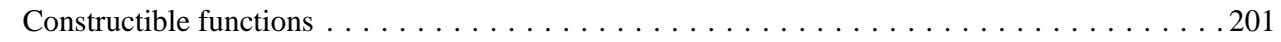

Semicanonical bases . . . . . . . . . . . . . . . . . . . . . . . . . . . . . . . 202

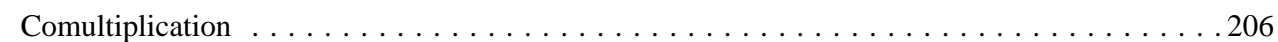

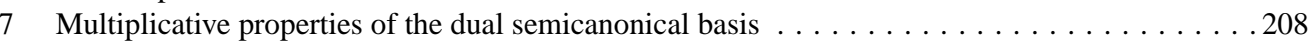

ANNALES SCIENTIFIQUES DE L'ÉCOLE NORMALE SUPÉRIEURE

0012-9593/02/@ 2005 Elsevier SAS. All rights reserved 


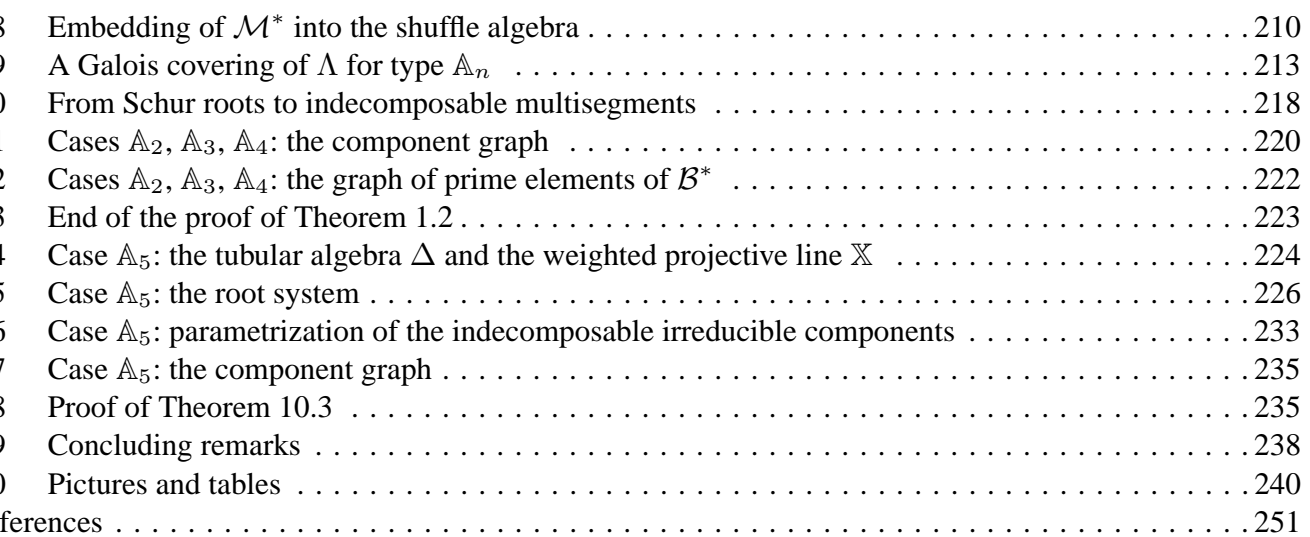

\section{Introduction}

1.1. Let $\mathfrak{g}$ be a simple Lie algebra of simply-laced type $\mathbb{A}, \mathbb{D}, \mathbb{E}$, and let $\mathfrak{n}$ be a maximal nilpotent subalgebra. Let $\mathcal{B}_{q}$ be the canonical basis of the quantum enveloping algebra $U_{q}(\mathfrak{n})$ $[29,37]$ and $\mathcal{B}_{q}^{*}$ the basis dual to $\mathcal{B}_{q}$. When $q$ tends to 1 , these two bases specialize to bases $\mathcal{B}$ and $\mathcal{B}^{*}$ of $U(\mathfrak{n})$ and $\mathbb{C}[N]$, respectively. Here $N$ stands for a maximal unipotent subgroup of a complex simple Lie group $G$ with Lie algebra $\mathfrak{g}$.

Let $I$ denote an indexing set for the simple roots of $\mathfrak{g}$. Given a finite-dimensional $I$-graded vector space $\mathbf{V}$ with graded dimension $|\mathbf{V}|$, we denote by $\Lambda_{\mathbf{V}}$ the corresponding nilpotent variety, see $[38, \S 12]$. This variety can be seen as the variety of modules over the preprojective algebra $\Lambda$ attached to the Dynkin diagram of $\mathfrak{g}$, with underlying vector space $\mathbf{V}$ [46].

For a variety $X$ let $\operatorname{Irr}(X)$ be the set of irreducible components of $X$. Lusztig has shown that there are natural bijections

$$
\begin{gathered}
\operatorname{Irr}\left(\Lambda_{\mathbf{V}}\right) \longrightarrow \mathcal{B}_{q}(|\mathbf{V}|) \quad\left(\text { resp. } \mathcal{B}_{q}^{*}(|\mathbf{V}|)\right) \\
Z \mapsto b_{Z} \quad\left(\text { resp. } b_{Z}^{*}\right)
\end{gathered}
$$

where $B_{q}(|\mathbf{V}|)$ (resp. $\left.B_{q}^{*}(|\mathbf{V}|)\right)$ is the subset of $\mathcal{B}_{q}$ (resp. $\mathcal{B}_{q}^{*}$ ) consisting of the elements of degree $|\mathbf{V}|$. Kashiwara and Saito [30] proved that the crystal basis of $U_{q}(\mathfrak{n})$ can be constructed geometrically in terms of these irreducible components (this was a conjecture of Lusztig).

This paper is motivated by several problems about the bases $\mathcal{B}_{q}$ and $\mathcal{B}_{q}^{*}$ and their relations with the varieties $\Lambda_{\mathrm{V}}$ and the preprojective algebra $\Lambda$.

1.2. One problem, which was first considered by Berenstein and Zelevinsky [4], is to study the multiplicative structure of the basis $\mathcal{B}_{q}^{*}$. Two elements $b_{1}^{*}$ and $b_{2}^{*}$ of $\mathcal{B}_{q}^{*}$ are called multiplicative if their product belongs to $\mathcal{B}_{q}^{*}$ up to a power of $q$. It was conjectured in [4] that $b_{1}^{*}$ and $b_{2}^{*}$ are multiplicative if and only if they $q$-commute. We refer to this as the BZ-conjecture. The conjecture was proved for types $\mathbb{A}_{2}$ and $\mathbb{A}_{3}$ [4], and it also holds for $\mathbb{A}_{4}$ [51].

More recently, Marsh and Reineke observed a strong relationship between the multiplicative structure of $\mathcal{B}_{q}^{*}$ and properties of the irreducible components of the varieties $\Lambda_{\mathrm{V}}$. They checked [42] that for $\mathfrak{g}$ of type $\mathbb{A}_{n}(n \leqslant 3)$, if the irreducible components $Z_{1} \subset \Lambda_{\mathbf{V}_{1}}$ and $Z_{2} \subset \Lambda_{\mathbf{V}_{2}}$ are the closures of the isomorphism classes of two indecomposable $\Lambda$-modules $x_{1}$ and $x_{2}$, then $b_{Z_{1}}^{*}$ and $b_{Z_{2}}^{*}$ are multiplicative if and only if $\operatorname{Ext}_{\Lambda}^{1}\left(x_{1}, x_{2}\right)=0$. This was verified by a caseby-case calculation, using the fact that for type $\mathbb{A}_{n}(n \leqslant 3)$ the preprojective algebra is of finite 
representation type, that is, it has only a finite number of isomorphism classes of indecomposable modules [15]. They also calculated many examples in type $\mathbb{A}_{4}$ and conjectured that this property still holds in this case (note that $\Lambda$ is again representation-finite for $\mathbb{A}_{4}$ ). But a conceptual explanation was still missing.

Let $Z_{1} \oplus Z_{2}$ denote the subset of $\Lambda_{\mathbf{V}_{1} \oplus \mathbf{V}_{2}}$ consisting of all $\Lambda$-modules $x$ isomorphic to $y_{1} \oplus y_{2}$ with $y_{1} \in Z_{1}$ and $y_{2} \in Z_{2}$. It follows from a general decomposition theory for irreducible components of varieties of modules developed in [14] that the condition $\operatorname{Ext}_{\Lambda}^{1}\left(x_{1}, x_{2}\right)=0$ for some $\left(x_{1}, x_{2}\right) \in Z_{1} \times Z_{2}$ is equivalent to $\overline{Z_{1} \oplus Z_{2}}$ being an irreducible component of $\Lambda_{\mathbf{V}_{1} \oplus \mathbf{V}_{2}}$.

In [31] counterexamples to the BZ-conjecture were found for all types other than $\mathbb{A}_{n}$ with $n \leqslant 4$. In particular in type $\mathbb{A}_{5}$, for a certain $\mathbf{V}$ of dimension 8 one can find an irreducible component $Z$ of $\Lambda_{\mathrm{V}}$ such that

$$
\left(b_{Z}^{*}\right)^{2}=q^{-2}\left(b_{Z^{\prime}}^{*}+b_{Z^{\prime \prime}}^{*}\right)
$$

where $Z^{\prime}=\overline{Z \oplus Z}$ and $Z^{\prime \prime}$ are two irreducible components of $\Lambda_{\mathbf{V} \oplus \mathbf{V}}$, see also [25]. This seems to be the smallest counterexample to the BZ-conjecture in type $\mathbb{A}$. Moreover, it also shows that the result of Marsh and Reineke does not generalize to $\mathbb{A}_{5}$. Note however that the BZ-conjecture was proved for large families of elements of $\mathcal{B}_{q}^{*}[8-10,33]$. For example, in type $\mathbb{A}$ it holds for quantum flag minors, and the reformulation in terms of direct sums of irreducible components is also valid [49].

So one would like to get a better understanding of the relationship between multiplicativity of elements of $\mathcal{B}_{q}^{*}$ and direct sum decompositions of irreducible components of varieties of $\Lambda$-modules.

1.3. Another interesting problem concerns the singular supports of the simple perverse sheaves used by Lusztig [37] to define the canonical basis $\mathcal{B}_{q}$. Let $Q$ be a Dynkin quiver, which is obtained from the Dynkin diagram of $\mathfrak{g}$ by choosing an orientation. Let $\operatorname{rep}(Q, \mathbf{V})$ be the affine space of representations of $Q$ with underlying finite-dimensional $I$-graded vector space $\mathbf{V}$. This is a finite union of isomorphism classes (or orbits) $\mathcal{O}$. In Lusztig's geometric construction, the elements of $\mathcal{B}_{q}(|\mathbf{V}|)$ are given by the perverse extensions $L_{\mathcal{O}}$ of the constant sheaves $\mathbb{C}_{\mathcal{O}}$ on the orbits $\mathcal{O}$. In [38] Lusztig considered the singular supports $S S\left(L_{\mathcal{O}}\right)$ of these sheaves and showed that they are unions of irreducible components of $\Lambda_{\mathrm{V}}$ (independent of the chosen orientation of the Dynkin diagram of $\mathfrak{g})$. He conjectured that in fact each $S S\left(L_{\mathcal{O}}\right)$ is irreducible, equal to the closure $\Lambda_{\mathcal{O}}$ of the conormal bundle of $\mathcal{O}$. Unexpectedly, Kashiwara and Saito [30] produced a counterexample to this conjecture. They exhibited two orbits $\mathcal{O}^{\prime}, \mathcal{O}^{\prime \prime}$ for type $\mathbb{A}_{5}$ such that

$$
S S\left(L_{\mathcal{O}^{\prime \prime}}\right)=\Lambda_{\mathcal{O}^{\prime}} \cup \Lambda_{\mathcal{O}^{\prime \prime}}
$$

The corresponding vectors $b_{\mathcal{O}^{\prime}}$ and $b_{\mathcal{O}^{\prime \prime}}$ of $\mathcal{B}_{q}$ have principal degree 16 , and apparently this is the smallest counterexample in type $\mathbb{A}$.

It turns out that this counterexample is dual to the counterexample above for $\mathcal{B}_{q}^{*}$, in the sense that $\Lambda_{\mathcal{O}^{\prime}}=Z^{\prime}$ and $\Lambda_{\mathcal{O}^{\prime \prime}}=Z^{\prime \prime}$, see [31, Remark 1]. One motivation for this paper was to find an explanation for this coincidence.

1.4. What makes these problems difficult is that, although the canonical basis reflects by definition the geometry of the varieties $\overline{\mathcal{O}} \subseteq \operatorname{rep}(Q, \mathbf{V})$, we want to relate it to the geometry of some other varieties, namely the irreducible components of the nilpotent varieties $\Lambda_{\mathbf{V}}$. It is natural to think of an intermediate object, that is, a basis reasonably close to the canonical basis, but directly defined in terms of the varieties $\Lambda_{\mathbf{V}}$. Lusztig [41] has constructed such a basis $\mathcal{S}=\left\{f_{Z}\right\}$ and called it the semicanonical basis. This is a basis of $U(\mathfrak{n})$ (not of the $q$-deformation 
$\left.U_{q}(\mathfrak{n})\right)$ which gives rise, like $\mathcal{B}$, to a basis in each irreducible highest weight $U(\mathfrak{g})$-module. Let $\mathcal{S}^{*}=\left\{\rho_{Z}\right\}$ denote the basis of $\mathbb{C}[N]$ dual to $\mathcal{S}$. Our first main result is the following:

THEOREM 1.1. - If $Z_{1} \subset \Lambda_{\mathbf{V}_{1}}$ and $Z_{2} \subset \Lambda_{\mathbf{V}_{2}}$ are irreducible components such that

$$
Z=\overline{Z_{1} \oplus Z_{2}}
$$

is an irreducible component of $\Lambda_{\mathbf{V}_{1} \oplus \mathbf{V}_{2}}$, then $\rho_{Z_{1}} \rho_{Z_{2}}=\rho_{Z}$.

In other words, the dual semicanonical basis $\mathcal{S}^{*}$ satisfies the multiplicative property which was expected to hold for the dual canonical basis $\mathcal{B}^{*}$.

An irreducible component $Z \in \operatorname{Irr}\left(\Lambda_{\mathbf{V}}\right)$ is called indecomposable if $Z$ contains a dense subset of indecomposable $\Lambda$-modules. By [14], every irreducible component $Z$ of $\Lambda_{\mathrm{V}}$ has a canonical decomposition

$$
Z=\overline{Z_{1} \oplus \cdots \oplus Z_{m}}
$$

where the $Z_{i} \subset \Lambda_{\mathbf{V}_{i}}$ are indecomposable irreducible components. Our theorem implies that

$$
\rho_{Z}=\rho_{Z_{1}} \cdots \rho_{Z_{m}}
$$

Hence $\mathcal{S}^{*}$ has a natural description as a collection of families of monomials in the elements indexed by indecomposable irreducible components. Such a description of $\mathcal{S}^{*}$ resembles the description of $\mathcal{B}^{*}$ for type $\mathbb{A}_{n}(n \leqslant 4)$ obtained by Berenstein and Zelevinsky.

1.5. So a natural question is how close are the bases $\mathcal{S}^{*}$ and $\mathcal{B}^{*}$ ? In type $\mathbb{A}$, Berenstein and Zelevinsky [4] proved that all minors of the triangular matrix of coordinate functions on $N$ belong to $\mathcal{B}^{*}$. We prove that they also belong to $\mathcal{S}^{*}$. Hence using [33,49], it follows that $\mathcal{S}^{*} \cap \mathcal{B}^{*}$ contains all multiplicative products of flag minors. However the two bases differ in general. More precisely we have:

THEOREM 1.2. - The bases $\mathcal{S}^{*}$ and $\mathcal{B}^{*}$ coincide if and only if $\mathfrak{g}$ is of type $\mathbb{A}_{n}$ with $n \leqslant 4$.

For example in type $\mathbb{A}_{5}$, we deduce from Eq. (1) and Theorem 1.1 that

$$
\rho_{Z^{\prime}}=b_{Z^{\prime}}^{*}+b_{Z^{\prime \prime}}^{*}
$$

(where for simplicity we use the same notation $b_{Z^{\prime}}^{*}$ and $b_{Z^{\prime \prime}}^{*}$ for the specializations at $q=1$ ). Nevertheless, since $\mathcal{S}^{*}$ and $\mathcal{B}^{*}$ have lots of elements in common, we get an explanation why the BZ-conjecture (or rather its reformulation in terms of irreducible components of varieties of $\Lambda$-modules) holds for large families of elements of $\mathcal{B}^{*}$.

Of course, by duality, these results also allow to compare the bases $\mathcal{S}$ and $\mathcal{B}$. In particular, returning to the example of [30], we can check that

$$
b_{\mathcal{O}^{\prime \prime}}=f_{\Lambda_{\mathcal{O}^{\prime}}}+f_{\Lambda_{\mathcal{O}^{\prime \prime}}}
$$

and this is probably the smallest example in type $\mathbb{A}$ for which the canonical and semicanonical bases differ. One may conjecture that, in general, the elements $f_{Z}$ occurring in the $\mathcal{S}$-expansion of $b_{\mathcal{O}} \in \mathcal{B}$ are indexed by the irreducible components $Z$ of $S S\left(L_{\mathcal{O}}\right)$, so that $S S\left(L_{\mathcal{O}}\right)$ is irreducible if and only if $b_{\mathcal{O}}=f_{\Lambda_{\mathcal{O}}}$. (There is a similar conjecture of Lusztig [40] for the "semicanonical basis" of the group algebra of a Weyl group obtained from the irreducible components of the Steinberg variety.) Assuming this conjecture we get an explanation of the relationship between the counterexamples to the conjectures of Berenstein-Zelevinsky and Lusztig.

$4^{\mathrm{e}}$ SÉRIE - TOME $38-2005-\mathrm{N}^{\circ} 2$ 
1.6. In the last part of the paper, we consider the first case which is not well understood, namely type $\mathbb{A}_{5}$. In this case, the preprojective algebra $\Lambda$ is representation-infinite, but it is still of tame representation type [15]. Motivated by our description of $\mathcal{S}^{*}$ in terms of indecomposable irreducible components of varieties of $\Lambda$-modules, we give a classification of the indecomposable irreducible components for the case $\mathbb{A}_{5}$. We also give an explicit criterion to decide when the closure of the direct sum of two such components is again an irreducible component. These results are deduced from [24], in which a general classification of irreducible components of varieties of modules over tubular algebras is developed. They are naturally formulated in terms of the Ringel bilinear form $\langle-,-\rangle$ of a convex subalgebra $\Delta$ of a Galois covering of $\Lambda$. The algebra $\Delta$ is a tubular algebra of type $(6,3,2)$ and the corresponding 10-dimensional infinite root system $R$ is an elliptic root system of type $\mathbb{E}_{8}^{(1,1)}$ in the classification of Saito [50], with a 2-dimensional lattice of imaginary roots. Note that the irreducible component $Z$ of Eq. (1) corresponds to a generator of this lattice. (This is an a posteriori justification for calling $b_{Z}^{*}$ an imaginary vector in [31].) The Ringel form $\langle-,-\rangle$ allows to define a distinguished Coxeter matrix $\Phi$ of order 6 acting on $R$. We prove the following:

THEOREM 1.3. - There is a one-to-one correspondence $r \mapsto Z(r)$ between the set of Schur roots of $R$ and the set of indecomposable irreducible components of the nilpotent varieties of type $\mathbb{A}_{5}$ which do not contain an indecomposable projective $\Lambda$-module. Moreover $\overline{Z\left(r_{1}\right) \oplus Z\left(r_{2}\right)}$ is an irreducible component if and only if the Schur roots $r_{1}$ and $r_{2}$ satisfy certain conditions which are all expressible in terms of $\langle-,-\rangle$ and $\Phi$.

We also explain how to translate from the language of roots to the language of multisegments, which form a natural indexing set of canonical and semicanonical bases in type $\mathbb{A}$.

1.7. The paper is organized as follows. In Section 2 we recall the general theory of varieties of modules. We explain a general decomposition theory for irreducible components of such varieties. This is followed in Section 3 by a short introduction to preprojective algebras. Then we recall the concept of a constructible function in Section 4. Following Lusztig [41], we review in Section 5 the definition of the semicanonical basis of $U(\mathfrak{n})$, which is obtained by realizing $U(\mathfrak{n})$ as an algebra $\mathcal{M}$ of constructible functions on the nilpotent varieties. In order to study the dual semicanonical basis and its multiplicative properties we also need to describe the natural comultiplication of $U(\mathfrak{n})$ in terms of $\mathcal{M}$. This was not done in [41], so we provide this description in Section 6. In Section 7 we introduce the dual semicanonical basis $\mathcal{S}^{*}$ of $\mathcal{M}^{*}$ and prove Theorem 1.1. Note that for this theorem we do not restrict ourselves to types $\mathbb{A}, \mathbb{D}, \mathbb{E}$, and only assume that $\mathfrak{n}$ is the positive part of a symmetric Kac-Moody Lie algebra. We end this section with the proof of the "only if" part of Theorem 1.2. In Section 8 we embed $\mathcal{M}^{*}$ into the shuffle algebra. This gives a practical way of computing elements of $\mathcal{S}^{*}$. We use this to prove that in type $\mathbb{A}$ all nonzero minors in the coordinate functions of $N$ belong to $\mathcal{S}^{*}$. In the rest of the paper we focus on the Dynkin cases $\mathbb{A}_{n}(n \leqslant 5)$. In Section 9 we consider a Galois covering $\widetilde{\Lambda}$ of the algebra $\Lambda$, with Galois group $\mathbb{Z}$, and we use it to calculate the Auslander-Reiten quiver of $\Lambda$ for $n \leqslant 4$. We also introduce an algebra $\Delta$ whose repetitive algebra is isomorphic to $\widetilde{\Lambda}$. For $n \leqslant 4$, $\Delta$ has finite representation type, while for $n=5$ it is a tubular algebra of tubular type $(6,3,2)$. In Section 10 we recall from [24] that the indecomposable irreducible components of $\Lambda$ are in oneto-one correspondence with the $\mathbb{Z}$-orbits of Schur roots of $\widetilde{\Lambda}$. We also describe the map which associates to such a Schur root the multisegment indexing the corresponding indecomposable irreducible component. The component graphs for the representation-finite cases $\mathbb{A}_{2}, \mathbb{A}_{3}$ and $\mathbb{A}_{4}$ are constructed in Section 11, and the corresponding graphs of prime elements of $\mathcal{B}^{*}$ are described in Section 12. In Section 13 we prove the "if" part of Theorem 1.2. All the remaining sections are devoted to the case $\mathbb{A}_{5}$. In Section 14 we relate the category of $\widetilde{\Lambda}$-modules to the 
category $\bmod (\Delta)$ of modules over the tubular algebra $\Delta$ and to the category $\operatorname{coh}(\mathbb{X})$ of coherent sheaves on a weighted projective line $\mathbb{X}$ of type $(6,3,2)$ in the sense of Geigle and Lenzing [23]. In Section 15 we consider the Grothendieck groups $K_{0}(\bmod (\Delta)) \simeq K_{0}(\operatorname{coh}(\mathbb{X})) \simeq \mathbb{Z}^{10}$. They are naturally endowed with a (non-symmetric) bilinear form $\langle-,-\rangle$ (the Ringel form) and a Coxeter matrix. This gives rise to an elliptic root system of type $\mathbb{E}_{8}^{(1,1)}$. We give an explicit description of its set of positive roots and of the subset $R_{S}^{+}$of Schur roots. In Section 16, we show that $R_{S}^{+}$naturally parametrizes the $\mathbb{Z}$-orbits of Schur roots of $\widetilde{\Lambda}$, hence also the indecomposable irreducible components of $\Lambda$. Then Section 17 describes the component graph of $\Lambda$ for type $\mathbb{A}_{5}$, thus making precise the statements of Theorem 1.3. Section 18 consists of the proof of Theorem 10.3. We conclude by noting the existence of similar results for type $\mathbb{D}_{4}$ and by pointing out some possible connections with the theory of cluster algebras of Fomin and Zelevinsky (Section 19). Section 20 contains a collection of pictures and tables to which we refer at various places in the text.

1.8. Throughout, we use the following conventions. If $f: M_{1} \rightarrow M_{2}$ and $g: M_{2} \rightarrow M_{3}$ are maps, then the composition is denoted by $g f: M_{1} \rightarrow M_{3}$. Similarly, if $\alpha: 1 \rightarrow 2$ and $\beta: 2 \rightarrow 3$ are arrows in a quiver, then the composition of $\alpha$ and $\beta$ is denoted by $\beta \alpha$.

Modules are always assumed to be left modules.

All vector spaces are over the field $\mathbb{C}$ of complex numbers.

We set $\mathbb{Q}^{+}=\{q \in \mathbb{Q} \mid q>0\}, \mathbb{Q}^{-}=\{q \in \mathbb{Q} \mid q<0\}$ and $\mathbb{Q}_{\infty}=\mathbb{Q} \cup\{\infty\}$. We also set $\mathbb{Z}^{+}=\{z \in \mathbb{Z} \mid z>0\}$ and $\mathbb{N}=\mathbb{Z}^{+} \cup\{0\}$.

\section{Varieties of modules}

2.1. A quiver is a quadruple $Q=\left(I, Q_{1}, s, e\right)$ where $I$ and $Q_{1}$ are sets with $I$ non-empty, and $s, e: Q_{1} \rightarrow I$ are maps such that $s^{-1}(i)$ and $e^{-1}(i)$ are finite for all $i \in I$. We call $I$ the set of vertices and $Q_{1}$ the set of arrows of $Q$. For an arrow $\alpha \in Q_{1}$ one calls $s(\alpha)$ the starting vertex and $e(\alpha)$ the end vertex of $\alpha$.

A path of length $t$ in $Q$ is a sequence $p=\alpha_{1} \alpha_{2} \cdots \alpha_{t}$ of arrows such that $s\left(\alpha_{i}\right)=e\left(\alpha_{i+1}\right)$ for $1 \leqslant i \leqslant t-1$. Set $s(p)=s\left(\alpha_{t}\right)$ and $e(p)=e\left(\alpha_{1}\right)$. Additionally, for each vertex $i \in I$ let $1_{i}$ be a path of length 0 . By $\mathbb{C} Q$ we denote the path algebra of $Q$, with basis the set of all paths in $Q$ and product given by concatenation. A relation for $Q$ is a linear combination

$$
\sum_{i=1}^{t} \lambda_{i} p_{i}
$$

where $\lambda_{i} \in \mathbb{C}$ and the $p_{i}$ are paths of length at least two in $Q$ with $s\left(p_{i}\right)=s\left(p_{j}\right)$ and $e\left(p_{i}\right)=e\left(p_{j}\right)$ for all $1 \leqslant i, j \leqslant t$. Thus, we can regard a relation as an element in $\mathbb{C} Q$.

An ideal $J$ in $\mathbb{C} Q$ is admissible if it is generated by a set of relations for $Q$. Note that this differs from the usual definition of an admissible ideal, where one also assumes that the factor algebra $\mathbb{C} Q / J$ is finite-dimensional.

2.2. A map $d: I \rightarrow \mathbb{N}$ such that $I \backslash d^{-1}(0)$ is finite is called a dimension vector for $Q$. We also write $d_{i}$ instead of $d(i)$, and we often use the notation $d=\left(d_{i}\right)_{i \in I}$. By $\mathbb{N}^{(I)}$ we denote the semigroup of dimension vectors for $Q$.

Let $\mathcal{V}_{\text {fin }}(I)$ be the category of finite-dimensional $I$-graded vector spaces. Thus, the objects of $\mathcal{V}_{\text {fin }}(I)$ are of the form $\mathbf{V}=\bigoplus_{i \in I} V_{i}$ where the $V_{i}$ are finite-dimensional vector spaces, and only finitely many of the $V_{i}$ are nonzero. We call $|\mathbf{V}|=\left(\operatorname{dim}\left(V_{i}\right)\right)_{i \in I}$ the dimension vector or

$4^{e}$ SÉRIE - TOME $38-2005-\mathrm{N}^{\circ} 2$ 
degree of $\mathbf{V}$. The morphisms in $\mathcal{V}_{\text {fin }}(I)$ are just linear maps respecting the grading. Direct sums in $\mathcal{V}_{\text {fin }}(I)$ are defined in the obvious way.

A representation of $Q$ with underlying vector space $\mathbf{V} \in \mathcal{V}_{\text {fin }}(I)$ is an element

$$
x=\left(x_{\alpha}\right)_{\alpha \in Q_{1}} \in \operatorname{rep}(Q, \mathbf{V})=\bigoplus_{\alpha \in Q_{1}} \operatorname{Hom}_{\mathbb{C}}\left(V_{s(\alpha)}, V_{e(\alpha)}\right)
$$

For a representation $x=\left(x_{\alpha}\right)_{\alpha \in Q_{1}} \in \operatorname{rep}(Q, \mathbf{V})$ and a path $p=\alpha_{1} \alpha_{2} \cdots \alpha_{t}$ in $Q$ set

$$
x_{p}=x_{\alpha_{1}} x_{\alpha_{2}} \cdots x_{\alpha_{t}} .
$$

Then $x$ satisfies a relation $\sum_{i=1}^{t} \lambda_{i} p_{i}$ if $\sum_{i=1}^{t} \lambda_{i} x_{p_{i}}=0$. If $R$ is a set of relations for $Q$, then let $\operatorname{rep}(Q, R, \mathbf{V})$ be the set of all representations $x \in \operatorname{rep}(Q, \mathbf{V})$ which satisfy all relations in $R$. This is a closed subvariety of $\operatorname{rep}(Q, \mathbf{V})$. Let $A$ be the algebra $\mathbb{C} Q / J$, where $J$ is the admissible ideal generated by $R$. Note that every element in $\operatorname{rep}(Q, R, \mathbf{V})$ can be naturally interpreted as an $A$-module structure on $\mathbf{V}$, so we shall also write

$$
\bmod (A, \mathbf{V})=\operatorname{rep}(Q, R, \mathbf{V})
$$

This is the affine variety of $A$-modules with underlying vector space $\mathbf{V}$. A dimension vector for $A$ is by definition the same as a dimension vector for $Q$, that is, an element of $\mathbb{N}^{(I)}$. For $x \in \bmod (A, \mathbf{V})$ we call $\operatorname{dim}(x)=|\mathbf{V}|$ the dimension vector of $x$.

2.3. Define $G_{\mathbf{V}}=\prod_{i \in I} \mathrm{GL}\left(V_{i}\right)$. This algebraic group acts on $\bmod (A, \mathbf{V})$ as follows. For $g=\left(g_{i}\right)_{i \in I} \in G_{\mathbf{V}}$ and $x=\left(x_{\alpha}\right)_{\alpha \in Q_{1}} \in \bmod (A, \mathbf{V})$ define

$$
g \cdot x=\left(x_{\alpha}^{\prime}\right)_{\alpha \in Q_{1}} \quad \text { where } x_{\alpha}^{\prime}=g_{e(\alpha)} x_{\alpha} g_{s(\alpha)}^{-1} .
$$

The $G_{\mathbf{V}}$-orbit of an $A$-module $x \in \bmod (A, \mathbf{V})$ is denoted by $\mathcal{O}(x)$. Two $A$-modules $x, y \in \bmod (A, \mathbf{V})$ are isomorphic if and only if they lie in the same orbit.

For a dimension vector $d$ for $A$ set

$$
\mathbf{V}^{d}=\bigoplus_{i \in I} \mathbb{C}^{d_{i}}, \quad \bmod (A, d)=\bmod \left(A, \mathbf{V}^{d}\right), \quad \operatorname{GL}(d)=G_{\mathbf{V}^{d}}
$$

Thus $\bmod (A,|\mathbf{V}|) \cong \bmod (A, \mathbf{V})$ for all $\mathbf{V} \in \mathcal{V}_{\text {fin }}(I)$. For this reason, we often do not distinguish between $\bmod (A,|\mathbf{V}|)$ and $\bmod (A, \mathbf{V})$. Any problems arising from this can be solved via the existence of an isomorphism between these two varieties which respects the group actions and the gradings.

2.4. By abuse of notation, we identify $i \in I$ with the dimension vector mapping $i$ to 1 and $j \neq i$ to 0 . If $\mathbf{V}$ is an $I$-graded vector space with $|\mathbf{V}|=i$, then the variety $\bmod (A, \mathbf{V})$ consists just of a single point and is denoted by $Z[i]$. The corresponding 1-dimensional $A$-module is denoted by $S_{i}$.

An element $x \in \bmod (A, \mathbf{V})$ is said to be nilpotent if there exists an $N \in \mathbb{Z}^{+}$such that for any path $p$ of length greater than $N$ we have $x_{p}=0 . \operatorname{By}_{\bmod ^{0}}(A, \mathbf{V})$ we denote the closed subset of nilpotent elements in $\bmod (A, \mathbf{V})$. The nilpotent elements are exactly the $A$-modules whose composition series contains only factors isomorphic to the simple modules $S_{i}, i \in I$. 
2.5. An irreducible component $Z \in \operatorname{Irr}(\bmod (A, \mathbf{V}))$ is called indecomposable if it contains a dense subset of indecomposable $A$-modules. Let $\operatorname{ind}(\operatorname{Irr}(\bmod (A, \mathbf{V})))$ be the set of indecomposable irreducible components of $\bmod (A, \mathbf{V})$. Let

$$
\operatorname{Irr}(A)=\bigcup_{d \in \mathbb{N}^{(I)}} \operatorname{Irr}(\bmod (A, d))
$$

be the set of all irreducible components of varieties of $A$-modules, and set

$$
\operatorname{ind}(\operatorname{Irr}(A))=\bigcup_{d \in \mathbb{N}^{(I)}} \operatorname{ind}(\operatorname{Irr}(\bmod (A, d))) .
$$

2.6. Let $Z_{i} \in \operatorname{Irr}\left(\bmod \left(A, \mathbf{V}^{i}\right)\right), 1 \leqslant i \leqslant m$, be irreducible components of $A$-modules, and set $\mathbf{V}=\mathbf{V}^{1} \oplus \cdots \oplus \mathbf{V}^{m}$. Let $Z_{1} \oplus \cdots \oplus Z_{m}$ be the set of modules in $\bmod (A, \mathbf{V})$, which are isomorphic to $x_{1} \oplus \cdots \oplus x_{m}$ with $x_{i} \in Z_{i}$ for all $i$. The closure $\overline{Z_{1} \oplus \cdots \oplus Z_{m}}$ is irreducible, but in general it is not an irreducible component. According to [14] any irreducible component $Z \in \operatorname{Irr}(A)$ has a decomposition

$$
Z=\overline{Z_{1} \oplus \cdots \oplus Z_{m}}
$$

for certain indecomposable irreducible components $Z_{i} \in \operatorname{ind}(\operatorname{Irr}(A))$. Moreover the components $Z_{1}, \ldots, Z_{m}$ are uniquely determined up to reordering. This is called the canonical decomposition of $Z$.

For irreducible components $Z^{\prime}$ and $Z^{\prime \prime}$ of $A$-modules define

$$
\operatorname{ext}_{A}^{1}\left(Z^{\prime}, Z^{\prime \prime}\right)=\min \left\{\operatorname{dim}_{\operatorname{Ext}_{A}^{1}}^{1}\left(x^{\prime}, x^{\prime \prime}\right) \mid\left(x^{\prime}, x^{\prime \prime}\right) \in Z^{\prime} \times Z^{\prime \prime}\right\} .
$$

This is the dimension of the extension group $\operatorname{Ext}_{A}^{1}\left(x^{\prime}, x^{\prime \prime}\right)$ for all $\left(x^{\prime}, x^{\prime \prime}\right)$ in a certain dense open subset of $Z^{\prime} \times Z^{\prime \prime}$. For irreducible components $Z_{i} \in \operatorname{Irr}(A)(1 \leqslant i \leqslant m)$ it is known that $\overline{Z_{1} \oplus \cdots \oplus Z_{m}}$ is an irreducible component if and only if $\operatorname{ext}_{A}^{1}\left(Z_{i}, Z_{j}\right)=0$ for all $i \neq j$, see [14].

The component graph $\mathcal{C}(A)$ of $A$ is defined as follows. The vertices of $\mathcal{C}(A)$ are the elements in $\operatorname{ind}(\operatorname{Irr}(A))$. There is an edge between vertices $Z^{\prime}$ and $Z^{\prime \prime}$ if and only if $\overline{Z^{\prime} \oplus Z^{\prime \prime}}$ is again an irreducible component, or equivalently if

$$
\operatorname{ext}_{A}^{1}\left(Z^{\prime}, Z^{\prime \prime}\right)=\operatorname{ext}_{A}^{1}\left(Z^{\prime \prime}, Z^{\prime}\right)=0
$$

\section{Preprojective algebras}

3.1. Assume that $Q=\left(I, Q_{1}, s, e\right)$ is a finite quiver without loops. (A loop is an arrow $\alpha$ with $s(\alpha)=e(\alpha)$, and $Q$ is finite if $I$ is finite. Note that this implies that $Q_{1}$ is finite as well.) The double quiver $\bar{Q}=\left(I, \overline{Q_{1}}, \bar{s}, \bar{e}\right)$ of $Q$ is obtained from $Q$ by adding for each arrow $\alpha \in Q_{1}$ an additional arrow $\alpha^{*}$. Define $\bar{s}(\alpha)=s(\alpha), \bar{e}(\alpha)=e(\alpha), \bar{s}\left(\alpha^{*}\right)=e(\alpha)$ and $\bar{e}\left(\alpha^{*}\right)=s(\alpha)$. For any $i \in I$ let

$$
r_{i}=\sum_{\alpha \in Q_{1}: s(\alpha)=i} \alpha^{*} \alpha-\sum_{\alpha \in Q_{1}: e(\alpha)=i} \alpha \alpha^{*}
$$

be the Gelfand-Ponomarev relation associated to $i$. This is a relation for $\bar{Q}$. The preprojective algebra $P(Q)$ corresponding to $Q$ is defined as

$$
P(Q)=\mathbb{C} \bar{Q} / J
$$

$4^{e}$ SÉRIE - TOME $38-2005-\mathrm{N}^{\circ} 2$ 
where $J$ is generated by the relations $r_{i}, i \in I$. These algebras were introduced and studied by Gelfand and Ponomarev, compare also [46].

3.2. For a preprojective algebra $\Lambda=P(Q)$ and some $\mathbf{V} \in \mathcal{V}_{\text {fin }}(I)$ set

$$
\Lambda_{\mathbf{V}}=\bmod ^{0}(P(Q), \mathbf{V}) .
$$

Lusztig proved that $\Lambda_{\mathbf{V}}$ has pure dimension $\operatorname{dim}(\operatorname{rep}(Q, \mathbf{V}))$, i.e. all irreducible components of $\Lambda_{\mathbf{V}}$ have dimension $\operatorname{dim}(\operatorname{rep}(Q, \mathbf{V}))$. Usually the varieties $\Lambda_{\mathbf{V}}$ are called nilpotent varieties. If $Q$ is a Dynkin quiver, then one might call them just preprojective varieties, since the nilpotency condition follows automatically in these cases, as shown by the next proposition.

PROPOSITION 3.1. - For a preprojective algebra $P(Q)$ the following are equivalent:

(a) $P(Q)$ is finite-dimensional;

(b) $\bmod ^{0}(P(Q), \mathbf{V})=\bmod (P(Q), \mathbf{V})$ for all $\mathbf{V} \in \mathcal{V}_{\text {fin }}(I)$;

(c) $Q$ is a Dynkin quiver.

The equivalence of (a) and (c) is classical (see for instance [44]). The implication (c) $\Longrightarrow$ (b) is proved by Lusztig [38, 14.2], and the converse by Crawley-Boevey [13].

3.3. The following remarkable property of preprojective algebras was proved in [12].

PRoposition 3.2. - For finite-dimensional modules $X$ and $Y$ over a preprojective algebra $\Lambda$ we have

$$
\operatorname{dim} \operatorname{Ext}_{\Lambda}^{1}(X, Y)=\operatorname{dim}_{\operatorname{Ext}_{\Lambda}^{1}(Y, X) .}
$$

Most preprojective algebras are of wild representation type. The following proposition lists the exceptional cases. We refer to [15] and [24] for further details.

PROPOSITION 3.3. - Let $P(Q)$ be a preprojective algebra. Then the following hold:

(a) $P(Q)$ is of finite representation type if and only if $Q$ is of Dynkin type $\mathbb{A}_{n}$ with $n \leqslant 4$;

(b) $P(Q)$ is of tame representation type if and only if $Q$ is of Dynkin type $\mathbb{A}_{5}$ or $\mathbb{D}_{4}$.

\section{Constructible functions}

4.1. Let $X$ be an algebraic variety over $\mathbb{C}$, endowed with its Zariski topology. A subset $A$ of $X$ is said to be constructible if it is a finite union of locally closed subsets. It is easy to see that if $X$ is irreducible and if we have a partition $X=A_{1} \sqcup \cdots \sqcup A_{k}$ into a finite number of constructible subsets, then there exists a unique $A_{i}$ containing a dense open subset of $X$.

A function $f: X \rightarrow \mathbb{C}$ is constructible if it is a finite $\mathbb{C}$-linear combination of characteristic functions $\mathbf{1}_{A}$ for various constructible subsets $A$. Equivalently, $f(X)$ is finite and $f^{-1}(m)$ is a constructible subset of $X$ for all $m \in \mathbb{C}$. The set of constructible functions on $X$ is denoted by $M(X)$. This is a $\mathbb{C}$-vector space.

4.2. If $Z$ is an irreducible component of $X$ and $f \in M(X)$, then

$$
Z=\bigsqcup_{m \in \mathbb{C}}\left(Z \cap f^{-1}(m)\right)
$$

is a finite partition into constructible subsets, hence there is a unique $m \in \mathbb{C}$ such that $Z \cap f^{-1}(m)$ contains a dense open subset of $Z$. In other words, a constructible function $f$ has to be constant 
on a dense open subset of each irreducible component $Z$ of $X$. We denote by

$$
\rho_{Z}: M(X) \rightarrow \mathbb{C}
$$

the linear form associating to $f$ its value on this dense open subset of $Z$.

4.3. By $\chi(A)$ we denote the Euler characteristic of a constructible subset $A$. If $A \cap B=\emptyset$, then $\chi(A \sqcup B)=\chi(A)+\chi(B)$. Hence $\chi$ can be regarded as a "measure" on the set of constructible subsets of $X$. For $f \in M(X)$ it is then natural to define

$$
\int_{x \in X} f(x)=\sum_{m \in \mathbb{C}} m \chi\left(f^{-1}(m)\right) \in \mathbb{C} .
$$

This is a linear form on $M(X)$. More generally, for a constructible subset $A$ of $X$ we write

$$
\int_{x \in A} f(x)=\sum_{m \in \mathbb{C}} m \chi\left(f^{-1}(m) \cap A\right) .
$$

\section{Semicanonical bases}

5.1. In this section we assume that $Q=\left(I, Q_{1}, s, e\right)$ is a finite quiver without loops, and as before for $\mathbf{V} \in \mathcal{V}_{\text {fin }}(I)$ let $\Lambda_{\mathbf{V}}=\bmod ^{0}(P(Q), \mathbf{V})$ be the corresponding nilpotent variety. We denote by $M\left(\Lambda_{\mathbf{V}}\right)^{G}$ the subspace of $M\left(\Lambda_{\mathbf{V}}\right)$ consisting of the constructible functions which are constant on the orbits of $G_{\mathbf{V}}$.

5.2. For $\mathbf{V}, \mathbf{V}^{\prime}, \mathbf{V}^{\prime \prime}$ such that $\left|\mathbf{V}^{\prime}\right|+\left|\mathbf{V}^{\prime \prime}\right|=|\mathbf{V}|$, Lusztig [41] defines a bilinear map

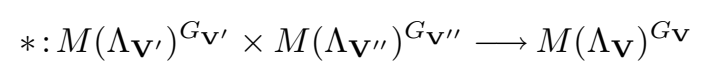

as follows. Let $x \in \Lambda_{\mathbf{V}}$. Define $V_{x}$ to be the variety of all $I$-graded subspaces $\mathbf{U}$ of $\mathbf{V}$ such that $x(\mathbf{U}) \subseteq \mathbf{U}$ and $|\mathbf{U}|=\left|\mathbf{V}^{\prime \prime}\right|$. In other words, $V_{x}$ is the variety of all $\Lambda$-submodules of $x$ with dimension vector $\left|\mathbf{V}^{\prime \prime}\right|$. For such a $\mathbf{U}$ let $x^{\prime} \in \Lambda_{\mathbf{V} / \mathbf{U}}$ and $x^{\prime \prime} \in \Lambda_{\mathbf{U}}$ be the elements induced by $x$, and let $\widetilde{x}^{\prime} \in \Lambda_{\mathbf{V}^{\prime}}$ and $\widetilde{x}^{\prime \prime} \in \Lambda_{\mathbf{V}^{\prime \prime}}$ be the elements obtained by transporting $x^{\prime}$ and $x^{\prime \prime}$ via some isomorphisms $\mathbf{V} / \mathbf{U} \stackrel{\sim}{\longrightarrow} \mathbf{V}^{\prime}$ and $\mathbf{U} \stackrel{\sim}{\longrightarrow} \mathbf{V}^{\prime \prime}$. For $f^{\prime} \in M\left(\Lambda_{\mathbf{V}^{\prime}}\right)^{G_{\mathbf{V}^{\prime}}}$ and $f^{\prime \prime} \in M\left(\Lambda_{\mathbf{V}^{\prime \prime}}\right)^{G} \mathbf{V}^{\prime \prime}$ define $\phi_{x}\left(f^{\prime}, f^{\prime \prime}\right): V_{x} \rightarrow \mathbb{C}$ by

$$
\phi_{x}\left(f^{\prime}, f^{\prime \prime}\right)(\mathbf{U})=f^{\prime}\left(\widetilde{x}^{\prime}\right) f^{\prime \prime}\left(\widetilde{x}^{\prime \prime}\right) .
$$

Following [41] define

$$
\left(f^{\prime} * f^{\prime \prime}\right)(x)=\int_{\mathbf{U} \in V_{x}} \phi_{x}\left(f^{\prime}, f^{\prime \prime}\right)(\mathbf{U}) .
$$

5.3. Let $\mathcal{O}^{\prime} \subset \Lambda_{\mathbf{V}^{\prime}}$ (resp. $\mathcal{O}^{\prime \prime} \subset \Lambda_{\mathbf{V}^{\prime \prime}}$ ) be a $G_{\mathbf{V}^{\prime}}$-orbit (resp. a $G_{\mathbf{V}^{\prime \prime}}$-orbit). For $x \in \Lambda_{\mathbf{V}}$ let $\mathcal{F}\left(\mathcal{O}^{\prime}, \mathcal{O}^{\prime \prime}, x\right)$ be the constructible subset of $V_{x}$ consisting of all $\Lambda$-submodules $y$ of $x$ of isomorphism type $\mathcal{O}^{\prime \prime}$ and such that $x / y$ has isomorphism type $\mathcal{O}^{\prime}$. The above definition yields

$$
\left(\mathbf{1}_{\mathcal{O}^{\prime}} * \mathbf{1}_{\mathcal{O}^{\prime \prime}}\right)(x)=\chi\left(\mathcal{F}\left(\mathcal{O}^{\prime}, \mathcal{O}^{\prime \prime}, x\right)\right)
$$

$4^{\mathrm{e}}$ SÉRIE - TOME $38-2005-\mathrm{N}^{\circ} 2$ 
Note that in general the variety $\Lambda_{\mathbf{V}}$ has infinitely many orbits. (Indeed, by Proposition 3.3 the algebra $\Lambda$ has in general infinite representation type, hence, by the validity of the second BrauerThrall conjecture (see [2]), it has in general an infinite number of non-isomorphic representations of a given dimension). Therefore the support of the function $\mathbf{1}_{\mathcal{O}^{\prime}} * \mathbf{1}_{\mathcal{O}^{\prime \prime}}$ may consist of an infinite number of orbits.

5.4. Let $\widetilde{\mathcal{M}}=\bigoplus_{\mathbf{V}} M\left(\Lambda_{\mathbf{V}}\right)^{G_{\mathbf{V}}}$, where $\mathbf{V}$ runs over the set of all isomorphism classes of vector spaces in $\mathcal{V}_{\text {fin }}(I)$. (For example, we can take $\mathbf{V} \in\left\{\mathbf{V}^{d} \mid d \in \mathbb{N}^{(I)}\right\}$.) The operation $*$ defines the structure of an $\mathbb{N}^{(I)}$-graded associative $\mathbb{C}$-algebra on $\widetilde{\mathcal{M}}$.

For $i \in I$, we recall that $Z[i]$ denotes the variety $\Lambda_{\mathbf{V}}$ where $|\mathbf{V}|=i$. (Z[i] is just a single point.) Following [41] define $\mathcal{M}$ to be the subalgebra of $(\widetilde{\mathcal{M}}, *)$ generated by the functions $\mathbf{1}_{Z[i]}, i \in I$. We set $\mathcal{M}_{\mathbf{V}}=\mathcal{M} \cap M\left(\Lambda_{\mathbf{V}}\right)^{G}$.

5.5. For two distinct vertices $i, j \in I$, let $a_{i j}$ denote the negative of the number of arrows $\alpha \in Q_{1}$ such that $\{s(\alpha), e(\alpha)\}=\{i, j\}$. Set also $a_{i i}=2(i \in I)$. Let $\mathfrak{g}$ be the symmetric KacMoody Lie algebra over $\mathbb{C}$ with Cartan matrix $\left(a_{i j}\right)_{i, j \in I}$. Let $\mathfrak{n}$ be a maximal nilpotent subalgebra of $\mathfrak{g}$, and let $U(\mathfrak{n})$ be its enveloping algebra. We denote by $e_{i}(i \in I)$ the Chevalley generators of $U(\mathfrak{n})$. The defining relations of $U(\mathfrak{n})$ are

$$
\sum_{k=0}^{-a_{i j}+1}(-1)^{k} e_{i}^{(k)} e_{j} e_{i}^{\left(-a_{i j}+1-k\right)}=0,
$$

where $e_{i}^{(k)}=e_{i}^{k} / k !$.

The algebra $U(\mathfrak{n})$ is $\mathbb{N}^{(I)}$-graded by assigning to $e_{i}$ the degree $i$. It is known that the number of irreducible components of Lusztig's nilpotent variety $\Lambda_{\mathrm{V}}$ is equal to the dimension of the homogeneous component of $U(\mathfrak{n})$ of degree $|\mathbf{V}|$. This was proved by Lusztig [38,39] when $\mathfrak{g}$ is of finite or affine type and by Kashiwara and Saito [30] in general.

5.6. Lusztig has proved that there is an algebra isomorphism

$$
\Psi: U(\mathfrak{n}) \rightarrow \mathcal{M}
$$

given by $\Psi\left(e_{i}\right)=\mathbf{1}_{Z[i]}$. To do this he constructed for every $I$-graded vector space $\mathbf{V}$ a $\mathbb{C}$-basis

$$
\left\{f_{Z} \mid Z \in \operatorname{Irr}\left(\Lambda_{\mathbf{V}}\right)\right\}
$$

of $\mathcal{M}_{\mathrm{V}}$, naturally labelled by the irreducible components of $\Lambda_{\mathrm{V}}$. Using the notation of Section 4.2 , it is characterized by

$$
\rho_{Z^{\prime}}\left(f_{Z}\right)=\delta_{Z, Z^{\prime}} \quad\left(Z, Z^{\prime} \in \operatorname{Irr}\left(\Lambda_{\mathbf{V}}\right)\right) .
$$

In other words, the function $f_{Z}$ is the unique element of $\mathcal{M}_{\mathbf{V}}$ equal to 1 on a dense open subset of $Z$ and equal to 0 on a dense open subset of any other irreducible component $Z^{\prime}$ of $\Lambda_{\mathrm{V}}$, see [41, Lemma 2.5].

The basis of $U(\mathfrak{n})$ obtained by transporting via $\Psi^{-1}$ the collection

$$
\bigcup_{\mathbf{V}}\left\{f_{Z} \mid Z \in \operatorname{Irr}\left(\Lambda_{\mathbf{V}}\right)\right\}
$$

ANNALES SCIENTIFIQUES DE L'ÉCOLE NORMALE SUPÉRIEURE 
where $\mathbf{V}$ ranges over the set of all isomorphism classes of vector spaces in $\mathcal{V}_{\text {fin }}(I)$, is called the semicanonical basis of $U(\mathfrak{n})$ and is denoted by $\mathcal{S}$.

Example 1. - Let $Q$ be the quiver with two vertices 1 and 2 and one arrow $\alpha: 1 \rightarrow 2$. Thus $Q$ is a Dynkin quiver of type $\mathbb{A}_{2}$.

(a) Let $\mathbf{V}=V_{1} \oplus V_{2}$ with $\operatorname{dim}\left(V_{1}\right)=\operatorname{dim} V_{2}=1$. Then

$$
\Lambda_{\mathbf{V}}=\{x=(a, b) \in \mathbb{C} \times \mathbb{C} \mid a b=0\} .
$$

The variety $\Lambda_{\mathbf{V}}$ has two irreducible components

$$
Z=\{(a, 0) \mid a \in \mathbb{C}\}, \quad Z^{\prime}=\{(0, b) \mid b \in \mathbb{C}\} .
$$

The group $G_{\mathbf{V}}=\mathbb{C}^{*} \times \mathbb{C}^{*}$ acts on $\Lambda_{\mathbf{V}}$ with three orbits $\{(0,0)\}, Z-\{(0,0)\}, Z^{\prime}-\{(0,0)\}$. We have

$$
f_{Z}=\mathbf{1}_{Z}=\mathbf{1}_{Z[1]} * \mathbf{1}_{Z[2]}, \quad f_{Z^{\prime}}=\mathbf{1}_{Z^{\prime}}=\mathbf{1}_{Z[2]} * \mathbf{1}_{Z[1]} .
$$

(b) Let $\mathbf{V}=V_{1} \oplus V_{2}$ with $\operatorname{dim}\left(V_{1}\right)=\operatorname{dim}\left(V_{2}\right)=2$. Then

$$
\operatorname{rep}(\bar{Q}, \mathbf{V})=M_{2}(\mathbb{C}) \times M_{2}(\mathbb{C})
$$

and $\Lambda_{\mathbf{V}} \subset \operatorname{rep}(\bar{Q}, \mathbf{V})$ has dimension 4 . The variety $\Lambda_{\mathbf{V}}$ has three irreducible components

$$
Z=\left\{x \in \Lambda_{\mathbf{V}} \mid \operatorname{rk}\left(x_{\alpha}\right) \leqslant 1, \operatorname{rk}\left(x_{\alpha^{*}}\right) \leqslant 1\right\}, \quad Z^{\prime}=\left\{x \mid x_{\alpha^{*}}=0\right\}, \quad Z^{\prime \prime}=\left\{x \mid x_{\alpha}=0\right\} .
$$

We have:

$$
\begin{gathered}
f_{Z}=\mathbf{1}_{Z}+\mathbf{1}_{\{(0,0)\}}=\frac{1}{2}\left(\mathbf{1}_{Z[1]} * \mathbf{1}_{Z[2]} * \mathbf{1}_{Z[2]} * \mathbf{1}_{Z[1]}\right)=\frac{1}{2}\left(\mathbf{1}_{Z[2]} * \mathbf{1}_{Z[1]} * \mathbf{1}_{Z[1]} * \mathbf{1}_{Z[2]}\right), \\
f_{Z^{\prime}}=\mathbf{1}_{Z^{\prime}}=\frac{1}{4}\left(\mathbf{1}_{Z[1]} * \mathbf{1}_{Z[1]} * \mathbf{1}_{Z[2]} * \mathbf{1}_{Z[2]}\right), \quad f_{Z^{\prime \prime}}=\mathbf{1}_{Z^{\prime \prime}}=\frac{1}{4}\left(\mathbf{1}_{Z[2]} * \mathbf{1}_{Z[2]} * \mathbf{1}_{Z[1]} * \mathbf{1}_{Z[1]}\right) .
\end{gathered}
$$

Note that $f_{Z} \neq \mathbf{1}_{Z}$ and $\mathbf{1}_{Z} \notin \mathcal{M}$.

5.7. Next, we consider composition series of modules over preprojective algebras. Let $\mathcal{X}$ denote the set of pairs $(\mathbf{i}, \mathbf{c})$ where $\mathbf{i}=\left(i_{1}, \ldots, i_{m}\right)$ is a sequence of elements of $I$ and $\mathbf{c}=\left(c_{1}, \ldots, c_{m}\right) \in\{0,1\}^{m}$. The integer $m$ is called the length of $(\mathbf{i}, \mathbf{c})$.

Given $(\mathbf{i}, \mathbf{c}) \in \mathcal{X}$ such that $\sum_{k} c_{k} i_{k}=|\mathbf{V}|$, we define a flag in $\mathbf{V}$ of type $(\mathbf{i}, \mathbf{c})$ as a sequence

$$
\mathfrak{f}=\left(\mathbf{V}=\mathbf{V}^{0} \supseteq \mathbf{V}^{1} \supseteq \cdots \supseteq \mathbf{V}^{m}=0\right)
$$

of graded subspaces of $\mathbf{V}$ such that

$$
\left|\mathbf{V}^{k-1} / \mathbf{V}^{k}\right|=c_{k} i_{k}
$$

for $k=1, \ldots, m$. Thus $\operatorname{dim} \mathbf{V}^{k-1} / \mathbf{V}^{k}$ is equal to 0 or 1 . So these are complete flags, with possible repetition of some subspaces. (It will be convenient below to allow such flags with repeated subspaces.) We denote by $\Phi_{\mathbf{i}, \mathbf{c}}$ the variety of flags of type $(\mathbf{i}, \mathbf{c})$. When $\left(c_{1}, \ldots, c_{m}\right)=$ $(1, \ldots, 1)$ (flags without repetition), we simply write $\Phi_{\mathbf{i}}$.

Let $x \in \Lambda_{\mathbf{V}}$. A flag $\mathfrak{f}$ is $x$-stable if $x\left(\mathbf{V}^{k}\right) \subseteq \mathbf{V}^{k}$ for all $k$. We denote by $\Phi_{\mathbf{i}, \mathbf{c}, x}$ (resp. $\left.\Phi_{\mathbf{i}, x}\right)$ the variety of $x$-stable flags of type (i,c) (resp. of type $\mathbf{i}$ ). Note that an $x$-stable flag is the same as a composition series of $x$ regarded as a $\Lambda$-module.

$4^{\mathrm{e}}$ SÉRIE - TOME $38-2005-\mathrm{N}^{\circ} 2$ 
5.8. $\quad$ For $(\mathbf{i}, \mathbf{c}) \in \mathcal{X}$ with $\sum_{k} c_{k} i_{k}=|\mathbf{V}|$, define

$$
d_{\mathbf{i}, \mathbf{c}}=\mathbf{1}_{Z\left[i_{1}\right]}^{c_{1}} * \cdots * \mathbf{1}_{Z\left[i_{m}\right]}^{c_{m}} \in \mathcal{M}_{\mathbf{V}} .
$$

If $c_{i}=1$ for all $k$, we simply write $d_{\mathbf{i}}$ instead of $d_{\mathbf{i}, \mathbf{c}}$. In general, $d_{\mathbf{i}, \mathbf{c}}=d_{\mathbf{j}}$ where $\mathbf{j}$ is the subword of $\mathbf{i}$ consisting of the letters $i_{k}$ for which $c_{k}=1$. By definition, the functions $d_{\mathbf{j}}$, where $\mathbf{j}$ runs over all words, span $\mathcal{M}$. The following lemma results immediately from the definition of the product $*$ of constructible functions.

LeMma 5.1. - Let $x \in \Lambda_{\mathrm{V}}$. We have

$$
d_{\mathbf{i}, \mathbf{c}}(x)=d_{\mathbf{j}}(x)=\chi\left(\Phi_{\mathbf{j}, x}\right) .
$$

Example 2. - Retain the notation of Example 1(b). Let $x=(a, b) \in \Lambda_{\mathbf{V}}$ be given by the following matrices (with respect to some fixed bases of $V_{1}$ and $V_{2}$ )

$$
a=\left(\begin{array}{ll}
0 & 0 \\
0 & 0
\end{array}\right) \in \operatorname{Hom}_{\mathbb{C}}\left(V_{1}, V_{2}\right), \quad b=\left(\begin{array}{ll}
1 & 0 \\
0 & 0
\end{array}\right) \in \operatorname{Hom}_{\mathbb{C}}\left(V_{2}, V_{1}\right) .
$$

Let us calculate $d_{(2,1,2,1)}(x)=\chi\left(\Phi_{(2,1,2,1), x}\right)$. To construct a flag

$$
\mathfrak{f}=\left(\mathbf{V} \supset \mathbf{V}^{1} \supset \mathbf{V}^{2} \supset \mathbf{V}^{3} \supset 0\right) \in \Phi_{(2,1,2,1), x}
$$

we first have to choose a line $\mathbf{V}^{3}$ in the 2-dimensional vector space $V_{1} \cap \operatorname{Socle}(x)=V_{1}$. We may take $\mathbf{V}^{3}$ to be

(a) the 1-dimensional image of $x$, or

(b) any line except this one.

In case (a) the module $x^{3}$ induced by $x$ in the quotient $\mathbf{V} / \mathbf{V}^{3}$ is the semisimple module. So we get

$$
\chi\left(\Phi_{(2,1,2), x^{3}}\right)=2 \cdot 1 \cdot 1 .
$$

In case (b), $x^{3}=\left(a^{3}, b^{3}\right)$ where

$$
a^{3}=\left(\begin{array}{l}
0 \\
0
\end{array}\right) \in \operatorname{Hom}_{\mathbb{C}}\left(V_{1} / \mathbf{V}^{3}, V_{2}\right), \quad b^{3}=\left(\begin{array}{ll}
1 & 0
\end{array}\right) \in \operatorname{Hom}_{\mathbb{C}}\left(V_{2}, V_{1} / \mathbf{V}^{3}\right) .
$$

and at the next stage $\mathbf{V}^{2} / \mathbf{V}^{3}$ must be taken as the kernel of $x^{3}$ (no choice), and $\mathbf{V}^{1}$ is also completely determined. Thus, in case (b) we get

$$
\chi\left(\Phi_{(2,1,2), x^{3}}\right)=1 \cdot 1 \cdot 1 .
$$

So finally,

$$
\chi\left(\Phi_{(2,1,2,1), x}\right)=2+1=3 .
$$

5.9. In this section we assume that $\mathfrak{g}$ is a simple finite-dimensional Lie algebra. Equivalently, $Q$ is a Dynkin quiver. Then $U(\mathfrak{n})$ has a PBW-basis $B_{Q}$ associated to this quiver $Q$. The image $\Psi\left(B_{Q}\right)$ is easy to describe. Let $\mathbf{V} \in \mathcal{V}_{\text {fin }}(I)$. The affine space $\operatorname{rep}(Q, \mathbf{V})$ can be regarded as a subset of $\Lambda_{\mathbf{V}}$ by identifying it to the set of $x \in \Lambda_{\mathbf{V}}$ with $x_{\alpha}=0$ for every $\alpha \in \bar{Q}_{1}-Q_{1}$. Clearly this is an irreducible component of $\Lambda_{\mathrm{V}}$. Moreover by our assumption it has finitely many $G_{\mathbf{V}}$-orbits. 
Lemma 5.2. - Let $\mathcal{O}$ be a $G_{\mathbf{V}}$-orbit in $\operatorname{rep}(Q, \mathbf{V})$. There exists a unique $\kappa_{\mathcal{O}} \in \mathcal{M}_{\mathbf{V}}$ whose restriction to $\operatorname{rep}(Q, \mathbf{V})$ is the characteristic function of $\mathcal{O}$. The collection of all $\kappa_{\mathcal{O}}$ where $\mathcal{O}$ runs through all $G_{\mathbf{V}}$-orbits in $\operatorname{rep}(Q, \mathbf{V})$ is equal to $\Psi\left(B_{Q}\right) \cap \mathcal{M}_{\mathbf{V}}$.

Proof. - By [38, 10.19, 12.12], the map from $\mathcal{M}_{\mathbf{V}}$ to $M(\operatorname{rep}(Q, \mathbf{V}))^{G_{\mathbf{V}}}$ sending $f$ to its restriction to $\operatorname{rep}(Q, \mathbf{V})$ is a vector space isomorphism. Moreover, the space

$$
\mathcal{H}_{Q}=\bigoplus_{\mathbf{V}} M(\operatorname{rep}(Q, \mathbf{V}))^{G_{\mathbf{V}}}
$$

(where $\mathbf{V}$ ranges over all isoclasses of vector spaces in $\mathcal{V}_{\text {fin }}(I)$ ) endowed with the image of the product $*$ is the geometric realization of the Hall algebra of $Q$ over $\mathbb{C}$ due to Schofield (see [38, 10.19]). In this setting, the PBW-basis is the basis of $\mathcal{H}_{Q}$ given by the characteristic functions of the $G_{\mathbf{V}}$-orbits in $\operatorname{rep}(Q, \mathbf{V})$. Thus the lemma is proved.

\section{Comultiplication}

The algebra $U(\mathfrak{n})$ is in fact a bialgebra, the comultiplication being defined by

$$
n \mapsto n \otimes 1+1 \otimes n \quad(n \in \mathfrak{n}) .
$$

In this section we describe the comultiplication $\Delta$ of $\mathcal{M}$ obtained by transporting this comultiplication via $\Psi$.

6.1. For $\mathbf{V}, \mathbf{V}^{\prime}, \mathbf{V}^{\prime \prime} \in \mathcal{V}_{\text {fin }}(I)$ such that $\left|\mathbf{V}^{\prime}\right|+\left|\mathbf{V}^{\prime \prime}\right|=|\mathbf{V}|$, define a linear map

$$
\operatorname{Res}_{\mathbf{V}^{\prime}, \mathbf{V}^{\prime \prime}}^{\mathbf{V}^{\prime \prime}}: M\left(\Lambda_{\mathbf{V}}\right)^{G \mathbf{V}} \longrightarrow M\left(\Lambda_{\mathbf{V}^{\prime}} \times \Lambda_{\mathbf{V}^{\prime \prime}}\right)^{G_{\mathbf{V}^{\prime}} \times G_{\mathbf{V}^{\prime \prime}}}
$$

as follows. We have $\mathbf{V} \stackrel{\sim}{\longrightarrow} \mathbf{V}^{\prime} \oplus \mathbf{V}^{\prime \prime}$, so given $x^{\prime} \in \Lambda_{\mathbf{V}^{\prime}}$ and $x^{\prime \prime} \in \Lambda_{\mathbf{V}^{\prime \prime}}$ we can regard $x^{\prime} \oplus x^{\prime \prime}$ as an element of $\Lambda_{\mathbf{V}}$. Here, $x^{\prime} \oplus x^{\prime \prime}$ denotes the direct sum of $x^{\prime}$ and $x^{\prime \prime}$ as endomorphisms of $\mathbf{V}^{\prime}$ and $\mathbf{V}^{\prime \prime}$, or equivalently as modules over the preprojective algebra $\Lambda$. For $f \in M_{\mathbf{V}}, x^{\prime} \in \Lambda_{\mathbf{V}^{\prime}}$ and $x^{\prime \prime} \in \Lambda_{\mathbf{V}^{\prime \prime}}$ set

$$
\left(\operatorname{Res}_{\mathbf{V}^{\prime}, \mathbf{V}^{\prime \prime}}^{\mathbf{V}} f\right)\left(x^{\prime}, x^{\prime \prime}\right)=f\left(x^{\prime} \oplus x^{\prime \prime}\right) .
$$

This is clearly a constructible function on $\Lambda_{\mathbf{V}^{\prime}} \times \Lambda_{\mathbf{V}^{\prime \prime}}$ which is constant on $G_{\mathbf{V}^{\prime}} \times G_{\mathbf{V}^{\prime \prime}}$-orbits.

6.2. Let $\mathbf{i}=\left(i_{1}, \ldots, i_{m}\right)$ with $\sum_{k} i_{k}=|\mathbf{V}|$. Let $\mathbf{V}=\mathbf{V}^{\prime} \oplus \mathbf{V}^{\prime \prime}, x^{\prime} \in \Lambda_{\mathbf{V}^{\prime}}, x^{\prime \prime} \in \Lambda_{\mathbf{V}^{\prime \prime}}$ and $x=x^{\prime} \oplus x^{\prime \prime} \in \Lambda_{\mathbf{V}}$.

LEMMA 6.1. - We have

$$
\left(\operatorname{Res}_{\mathbf{V}^{\prime}, \mathbf{V}^{\prime \prime}}^{\mathbf{V}} d_{\mathbf{i}}\right)\left(x^{\prime}, x^{\prime \prime}\right)=\sum_{\left(\mathbf{c}^{\prime}, \mathbf{c}^{\prime \prime}\right)} d_{\mathbf{i}, \mathbf{c}^{\prime}}\left(x^{\prime}\right) d_{\mathbf{i}, \mathbf{c}^{\prime \prime}}\left(x^{\prime \prime}\right)
$$

where the sum is over all pairs $\left(\mathbf{c}^{\prime}, \mathbf{c}^{\prime \prime}\right)$ of sequences in $\{0,1\}^{m}$ such that

$$
\sum_{k=0}^{m} c_{k}^{\prime} i_{k}=\left|\mathbf{V}^{\prime}\right|, \quad \sum_{k=0}^{m} c_{k}^{\prime \prime} i_{k}=\left|\mathbf{V}^{\prime \prime}\right|, \quad c_{k}^{\prime}+c_{k}^{\prime \prime}=1(0 \leqslant k \leqslant m) .
$$

$4^{e}$ SÉRIE - TOME $38-2005-\mathrm{N}^{\circ} 2$ 
Proof. - By definition we have

$$
\left(\operatorname{Res}_{\mathbf{V}^{\prime}, \mathbf{V}^{\prime \prime}}^{\mathbf{V}} d_{\mathbf{i}}\right)\left(x^{\prime}, x^{\prime \prime}\right)=d_{\mathbf{i}}(x)=\chi\left(\Phi_{\mathbf{i}, x}\right) .
$$

To $\mathfrak{f}=\left(\mathbf{V}^{l}\right)_{0 \leqslant l \leqslant m} \in \Phi_{\mathbf{i}, x}$, we associate the pair of flags

$$
\mathfrak{f}^{\prime}=\left(\mathbf{V}^{l} /\left(\mathbf{V}^{\prime \prime} \cap \mathbf{V}^{l}\right)\right)_{0 \leqslant l \leqslant m}, \quad \mathfrak{f}^{\prime \prime}=\left(\mathbf{V}^{l} \cap \mathbf{V}^{\prime \prime}\right)_{0 \leqslant l \leqslant m} .
$$

We regard $\mathfrak{f}^{\prime \prime}$ as a flag in $\mathbf{V}^{\prime \prime}$, and $\mathfrak{f}^{\prime}$ as a flag in $\mathbf{V}^{\prime}$ by identifying $\mathbf{V}^{\prime}$ with $\mathbf{V} / \mathbf{V}^{\prime \prime}$. Clearly, we have $\mathfrak{f}^{\prime} \in \Phi_{\mathbf{i}, \mathbf{c}^{\prime}, x^{\prime}}$ and $\mathfrak{f}^{\prime \prime} \in \Phi_{\mathbf{i}, \mathbf{c}^{\prime \prime}, x^{\prime \prime}}$ for some sequences $\mathbf{c}^{\prime}, \mathbf{c}^{\prime \prime}$ in $\{0,1\}^{m}$ satisfying the conditions (5). Let $W_{1}$ denote the set of pairs $\left(\mathbf{c}^{\prime}, \mathbf{c}^{\prime \prime}\right)$ satisfying (5). For $\left(\mathbf{c}^{\prime}, \mathbf{c}^{\prime \prime}\right) \in W_{1}$, let $\Phi_{\mathbf{i}, x}\left(\mathbf{c}^{\prime}, \mathbf{c}^{\prime \prime}\right)$ be the subset of $\Phi_{\mathbf{i}, x}$ consisting of those $\mathfrak{f}$ for which $\left(\mathfrak{f}^{\prime}, \mathfrak{f}^{\prime \prime}\right) \in \Phi_{\mathbf{i}, \mathbf{c}^{\prime}, x^{\prime}} \times \Phi_{\mathbf{i}, \mathbf{c}^{\prime \prime}, x^{\prime \prime}}$. Then clearly we have a finite partition

$$
\Phi_{\mathbf{i}, x}=\bigsqcup_{\left(\mathbf{c}^{\prime}, \mathbf{c}^{\prime \prime}\right) \in W_{2}} \Phi_{\mathbf{i}, x}\left(\mathbf{c}^{\prime}, \mathbf{c}^{\prime \prime}\right)
$$

where $W_{2} \subseteq W_{1}$ consists of the pairs $\left(\mathbf{c}^{\prime}, \mathbf{c}^{\prime \prime}\right)$ such that $\Phi_{\mathbf{i}, x}\left(\mathbf{c}^{\prime}, \mathbf{c}^{\prime \prime}\right)$ is nonempty.

Now for $\left(\mathbf{c}^{\prime}, \mathbf{c}^{\prime \prime}\right) \in W_{2}$, the map $\alpha\left(\mathbf{c}^{\prime}, \mathbf{c}^{\prime \prime}\right): \Phi_{\mathbf{i}, x}\left(\mathbf{c}^{\prime}, \mathbf{c}^{\prime \prime}\right) \longrightarrow \Phi_{\mathbf{i}, \mathbf{c}^{\prime}, x^{\prime}} \times \Phi_{\mathbf{i}, \mathbf{c}^{\prime \prime}, x^{\prime \prime}}$ sending $f$ to $\left(\mathfrak{f}^{\prime}, \mathfrak{f}^{\prime \prime}\right)$ is a vector bundle, see [38, Lemma 4.4]. Hence,

$$
\chi\left(\Phi_{\mathbf{i}, x}\left(\mathbf{c}^{\prime}, \mathbf{c}^{\prime \prime}\right)\right)=\chi\left(\Phi_{\mathbf{i}, \mathbf{c}^{\prime}, x^{\prime}} \times \Phi_{\mathbf{i}, \mathbf{c}^{\prime \prime}, x^{\prime \prime}}\right)=\chi\left(\Phi_{\mathbf{i}, \mathbf{c}^{\prime}, x^{\prime}}\right) \chi\left(\Phi_{\mathbf{i}, \mathbf{c}^{\prime \prime}, x^{\prime \prime}}\right)
$$

and

$$
\left(\operatorname{Res}_{\mathbf{V}^{\prime}, \mathbf{V}^{\prime \prime}}^{\mathbf{V}} d_{\mathbf{i}}\right)\left(x^{\prime}, x^{\prime \prime}\right)=\chi\left(\Phi_{\mathbf{i}, x}\right)=\sum_{\left(\mathbf{c}^{\prime}, \mathbf{c}^{\prime \prime}\right) \in W_{2}} \chi\left(\Phi_{\mathbf{i}, \mathbf{c}^{\prime}, x^{\prime}}\right) \chi\left(\Phi_{\mathbf{i}, \mathbf{c}^{\prime \prime}, x^{\prime \prime}}\right) .
$$

On the other hand,

$$
\sum_{\left(\mathbf{c}^{\prime}, \mathbf{c}^{\prime \prime}\right) \in W_{1}} d_{\mathbf{i}, \mathbf{c}^{\prime}}\left(x^{\prime}\right) d_{\mathbf{i}, \mathbf{c}^{\prime \prime}}\left(x^{\prime \prime}\right)=\sum_{\left(\mathbf{c}^{\prime}, \mathbf{c}^{\prime \prime}\right) \in W_{1}} \chi\left(\Phi_{\mathbf{i}, \mathbf{c}^{\prime}, x^{\prime}}\right) \chi\left(\Phi_{\mathbf{i}, \mathbf{c}^{\prime \prime}, x^{\prime \prime}}\right),
$$

and it only remains to prove that $W_{1}=W_{2}$. Clearly $W_{2} \subseteq W_{1}$, so let $\left(\mathbf{c}^{\prime}, \mathbf{c}^{\prime \prime}\right) \in W_{1}$. Let $\mathfrak{f}^{\prime} \in \Phi_{\mathbf{i}, \mathbf{c}^{\prime}, x^{\prime}}$ and $\mathfrak{f}^{\prime \prime} \in \Phi_{\mathbf{i}, \mathbf{c}^{\prime \prime}, x^{\prime \prime}}$. The assumption $x=x^{\prime} \oplus x^{\prime \prime}$ implies that $\Phi_{\mathbf{i}, x}\left(\mathbf{c}^{\prime}, \mathbf{c}^{\prime \prime}\right)$ is nonempty. Indeed, the flag $\mathfrak{f}$ in $\mathbf{V}$ whose $k$ th subspace is the direct sum of the $k$ th subspaces of $\mathfrak{f}^{\prime}$ and $\mathfrak{f}^{\prime \prime}$ is $x$-stable and by construction $\mathfrak{f} \in \Phi_{\mathbf{i}, x}\left(\mathbf{c}^{\prime}, \mathbf{c}^{\prime \prime}\right)$. So $\left(\mathbf{c}^{\prime}, \mathbf{c}^{\prime \prime}\right) \in W_{2}$ and the lemma is proved.

6.3. By Lemma 6.1, the map $\operatorname{Res}_{\mathbf{V}^{\prime}, \mathbf{V}^{\prime \prime}}^{\mathbf{V}}$ induces a linear map from $\mathcal{M}_{\mathbf{V}}$ to $\mathcal{M}_{\mathbf{V}^{\prime}} \otimes \mathcal{M}_{\mathbf{V}^{\prime \prime}}$, given by

$$
d_{\mathbf{i}} \mapsto \sum_{\left(\mathbf{c}^{\prime}, \mathbf{c}^{\prime \prime}\right)} d_{\mathbf{i}, \mathbf{c}^{\prime}} \otimes d_{\mathbf{i}, \mathbf{c}^{\prime \prime}},
$$

where the pairs $\left(\mathbf{c}^{\prime}, \mathbf{c}^{\prime \prime}\right)$ satisfy (5). Taking direct sums, we obtain a linear map

$$
\mathcal{M}_{\mathbf{V}} \longrightarrow \bigoplus_{\mathbf{V}^{\prime}, \mathbf{V}^{\prime \prime}} \mathcal{M}_{\mathbf{V}^{\prime}} \otimes \mathcal{M}_{\mathbf{V}^{\prime \prime}}
$$

the sum being over all isomorphism types $\mathrm{V}^{\prime}$ and $\mathbf{V}^{\prime \prime}$ of $I$-graded vector spaces such that $\left|\mathbf{V}^{\prime}\right|+\left|\mathbf{V}^{\prime \prime}\right|=|\mathbf{V}|$. Taking direct sums over all isomorphism types $\mathbf{V}$, we get a linear map

$$
\Delta: \mathcal{M} \longrightarrow \mathcal{M} \otimes \mathcal{M}
$$

ANNALES SCIENTIFIQUES DE L'ÉCOLE NORMALE SUPÉRIEURE 
Since $\left(x^{\prime} \oplus x^{\prime \prime}\right) \oplus x^{\prime \prime \prime}=x^{\prime} \oplus\left(x^{\prime \prime} \oplus x^{\prime \prime \prime}\right), \Delta$ is coassociative. Since $x^{\prime} \oplus x^{\prime \prime} \simeq x^{\prime \prime} \oplus x^{\prime}, \Delta$ is cocommutative.

Lemma 6.1 shows that $\Delta$ is multiplicative on the elements $d_{\mathbf{i}}$, that is, for $\mathbf{i}=\left(i_{1}, \ldots, i_{m}\right)$

$$
\Delta\left(d_{\mathbf{i}}\right)=\Delta\left(d_{i_{1}}\right) * \cdots * \Delta\left(d_{i_{m}}\right)=\left(d_{i_{1}} \otimes 1+1 \otimes d_{i_{1}}\right) * \cdots *\left(d_{i_{m}} \otimes 1+1 \otimes d_{i_{m}}\right),
$$

where the product in $\mathcal{M} \otimes \mathcal{M}$ is defined by

$$
\left(f_{1} \otimes f_{2}\right) *\left(g_{1} \otimes g_{2}\right)=\left(f_{1} * g_{1}\right) \otimes\left(f_{2} * g_{2}\right) .
$$

Proposition 6.2. - Under the algebra isomorphism $\Psi^{-1}: \mathcal{M} \longrightarrow U(\mathfrak{n})$, the map $\Delta$ gets identified with the standard comultiplication of $U(\mathfrak{n})$.

Proof. - Eq. (6) shows that $\Delta$ is an algebra homomorphism $(\mathcal{M}, *) \rightarrow(\mathcal{M} \otimes \mathcal{M}, *)$. Moreover the generators $d_{(i)}=\mathbf{1}_{Z[i]}=\Psi\left(e_{i}\right)$ are clearly primitive. The result follows.

\section{Multiplicative properties of the dual semicanonical basis}

7.1. The vector space $\mathcal{M}$ is $\mathbb{N}^{(I)}$-graded, with finite-dimensional homogeneous components. Let $\mathcal{M}^{*}$ denote its graded dual. Given an $I$-graded space $\mathbf{V}$ and an irreducible component $Z$ of $\Lambda_{\mathrm{V}}$, we have defined a linear form $\rho_{Z}$ on $M\left(\Lambda_{\mathrm{V}}\right)$, see Section 4.2. We shall also denote by $\rho_{Z}$ the element of $\mathcal{M}^{*}$ obtained by restricting $\rho_{Z}$ to $\mathcal{M}_{\mathbf{V}}$ and then extending by 0 on all $\mathcal{M}_{\mathbf{V}^{\prime}}$ with $\left|\mathbf{V}^{\prime}\right| \neq|\mathbf{V}|$. Note that by (4), the basis of $\mathcal{M}^{*}$ dual to the semicanonical basis $\left\{f_{Z}\right\}$ is nothing but $\left\{\rho_{Z}\right\}$. From now on $\mathcal{S}^{*}=\left\{\rho_{Z}\right\}$ will be called the dual semicanonical basis of $\mathcal{M}^{*}$.

Lemma 7.1. - For $Z \in \operatorname{Irr}\left(\Lambda_{\mathbf{V}}\right)$ there exists an open dense $G_{\mathbf{V}^{-}}$-stable subset $O_{Z} \subset Z$ such that for all $f \in \mathcal{M}_{\mathbf{V}}$ and all $x \in O_{Z}$ we have $\rho_{Z}(f)=f(x)$.

Proof. - For a given $f$, this follows from Section 4.2. Moreover, there exists such an open set simultaneously for all $f$ because $\mathcal{M}_{\mathrm{V}}$ is finite-dimensional.

7.2. For $x \in \Lambda_{\mathbf{V}}$ define the delta-function $\delta_{x} \in \mathcal{M}^{*}$ by $\delta_{x}(f)=f(x),(f \in \mathcal{M})$. We then have

$$
\delta_{x}=\rho_{Z} \quad\left(x \in O_{Z}\right)
$$

The next lemma follows immediately.

Lemma 7.2. - Let $Z \in \operatorname{Irr}\left(\Lambda_{\mathbf{V}}\right)$ and suppose that the orbit of $x \in Z$ is open dense. Then $\rho_{Z}=\delta_{x}$.

Let - denote the multiplication of $\mathcal{M}^{*}$ dual to the comultiplication $\Delta$ of $\mathcal{M}$.

LEMmA 7.3. - Let $x_{1} \in \Lambda_{\mathbf{V}_{1}}$ and $x_{2} \in \Lambda_{\mathbf{V}_{2}}$. We have $\delta_{x_{1}} \cdot \delta_{x_{2}}=\delta_{x_{1} \oplus x_{2}}$.

Proof. - For $f \in \mathcal{M}$, one has

$$
\left(\delta_{x_{1}} \cdot \delta_{x_{2}}\right)(f)=\left(\delta_{x_{1}} \otimes \delta_{x_{2}}\right)(\Delta(f))=\Delta(f)\left(x_{1}, x_{2}\right)=f\left(x_{1} \oplus x_{2}\right)=\delta_{x_{1} \oplus x_{2}}(f) .
$$

Lemma 7.4. - Suppose that $Z=\overline{Z_{1} \oplus Z_{2}}$ is an irreducible component of $\Lambda_{\mathbf{V}}$. Then there exists $x \in O_{Z}$ of the form $x=x_{1} \oplus x_{2}$ with $x_{1} \in O_{Z_{1}}$ and $x_{2} \in O_{Z_{2}}$.

$4^{\text {e }}$ SÉRIE - TOME $38-2005-\mathrm{N}^{\circ} 2$ 
Proof. - The direct sum $Z_{1} \oplus Z_{2}$ is the image of the morphism

$$
\theta: G_{\mathbf{V}} \times Z_{1} \times Z_{2} \longrightarrow Z
$$

defined by

$$
\theta\left(g, x_{1}, x_{2}\right)=g \cdot\left(x_{1} \oplus x_{2}\right) .
$$

Since $O_{Z_{1}}$ (resp. $O_{Z_{2}}$ ) is open dense in $Z_{1}$ (resp. in $Z_{2}$ ), the subset $G_{\mathbf{V}} \times O_{Z_{1}} \times O_{Z_{2}}$ is open dense in $G_{\mathbf{V}} \times Z_{1} \times Z_{2}$. Now, since $\theta$ is a dominant morphism between irreducible varieties, the image under $\theta$ of $G_{\mathbf{V}} \times O_{Z_{1}} \times O_{Z_{2}}$ contains a dense open subset of $Z$, hence it has a nonempty intersection with $O_{Z}$. Since both $\theta\left(G_{\mathbf{V}} \times O_{Z_{1}} \times O_{Z_{2}}\right)$ and $O_{Z}$ are $G_{\mathbf{V}^{-}}$-stable we can find $x$ in their intersection of the form $x=x_{1} \oplus x_{2}$ with $x_{1} \in O_{Z_{1}}$ and $x_{2} \in O_{Z_{2}}$.

7.3. We can now give the proof of Theorem 1.1.

Proof of Theorem 1.1. - Choose $x, x_{1}, x_{2}$ as in Lemma 7.4. Then Lemmas 7.1 and 7.3 yield

$$
\rho_{Z_{1}} \cdot \rho_{Z_{2}}=\delta_{x_{1}} \cdot \delta_{x_{2}}=\delta_{x_{1} \oplus x_{2}}=\delta_{x}=\rho_{Z} .
$$

COROLlary 7.5. - Let $Z=\overline{Z_{1} \oplus \cdots \oplus Z_{m}}$ be the canonical decomposition of the irreducible component $Z$ of $\Lambda_{\mathbf{V}}$. The dual semicanonical basis vector $\rho_{Z}$ factorizes as

$$
\rho_{Z}=\rho_{Z_{1}} \cdots \rho_{Z_{m}}
$$

Proof. - For $m=2$ this follows from Theorem 1.1. Assume that $m>2$. By [14]

$$
Z^{\prime}=\overline{Z_{1} \oplus \cdots \oplus Z_{m-1}}
$$

is an irreducible component. Moreover

$$
\overline{Z^{\prime} \oplus Z_{m}}=Z,
$$

so by Theorem 1.1 we get $\rho_{Z}=\rho_{Z^{\prime}} \cdot \rho_{Z_{m}}$. The result follows by induction on $m$.

The factorization given by Corollary 7.5 will be called the canonical factorization of $\rho_{Z}$.

7.4. We shall now deduce from Theorem 1.1 the proof of the "only if" part of Theorem 1.2.

THEOREM 7.6. - Let $\mathfrak{g}$ be of type $\mathbb{A}_{n}(n \geqslant 5), \mathbb{D}_{n}(n \geqslant 4), \mathbb{E}_{6}, \mathbb{E}_{7}$ or $\mathbb{E}_{8}$. Then, the bases $\mathcal{B}^{*}$ and $\mathcal{S}^{*}$ do not coincide.

Proof. - Assume first that $\mathfrak{g}$ is of type $\mathbb{A}_{5}$ or $\mathbb{D}_{4}$. Then the preprojective algebra $\Lambda$ is of tame representation type. In this case, we have $\operatorname{ext}_{\Lambda}^{1}(Z, Z)=0$ for any irreducible component $Z$ of $\Lambda_{\mathrm{V}}$, see $[24,25]$. Therefore by Theorem 1.1 and [14] the square of any vector of $\mathcal{S}^{*}$ belongs to $\mathcal{S}^{*}$.

On the other hand, it was shown in [31] that for the cases $\mathbb{A}_{5}$ and $\mathbb{D}_{4}$ there exist elements of $\mathcal{B}^{*}$ whose square is not in $\mathcal{B}^{*}$. They are called imaginary vectors of $\mathcal{B}^{*}$. This shows that $\mathcal{B}^{*}$ and $\mathcal{S}^{*}$ are different in these cases.

Now if $\mathfrak{g}$ is not of type $\mathbb{A}_{n}$ with $n \leqslant 4$, then the Dynkin diagram of $\mathfrak{g}$ contains a subdiagram of type $\mathbb{A}_{5}$ or $\mathbb{D}_{4}$, and the result follows from the cases $\mathbb{A}_{5}$ and $\mathbb{D}_{4}$.

In the next sections we shall prepare some material for the proof of the "if" part of Theorem 1.2, to be given in Section 13. 


\section{Embedding of $\mathcal{M}^{*}$ into the shuffle algebra}

We describe a natural embedding of $\mathcal{M}^{*}$ into the shuffle algebra. This is then used to describe a certain family of elements of $\mathcal{S}^{*}$ in type $\mathbb{A}_{n}$.

8.1. Let $F=\mathbb{C}\langle I\rangle$ be the free associative algebra over $\mathbb{C}$ generated by $I$. A monomial in $F$ is called a word. This is nothing else than a sequence $\mathbf{i}=\left(i_{1}, \ldots, i_{k}\right)$ in $I$. Let

$$
\pi: F \longrightarrow \mathcal{M}
$$

be the surjective algebra homomorphism given by $\pi(i)=\mathbf{1}_{Z[i]}$, and more generally by $\pi(\mathbf{i})=d_{\mathbf{i}}$. Let $F^{*}$ denote the graded dual of $F$. We thus get an embedding of vector spaces

$$
\pi^{*}: \mathcal{M}^{*} \longrightarrow F^{*}
$$

Let $\{w[\mathbf{i}]\}$ denote the basis of $F^{*}$ dual to the basis $\{\mathbf{i}\}$ of words in $F$. Let $\sigma \in \mathcal{M}^{*}$. We have

$$
\pi^{*}(\sigma)=\sum_{\mathbf{i}} \pi^{*}(\sigma)(\mathbf{i}) w[\mathbf{i}]=\sum_{\mathbf{i}} \sigma(\pi(\mathbf{i})) w[\mathbf{i}]=\sum_{\mathbf{i}} \sigma\left(d_{\mathbf{i}}\right) w[\mathbf{i}]
$$

By Lemma 5.1, we obtain in particular

$$
\pi^{*}\left(\delta_{x}\right)=\sum_{\mathbf{i}} \chi\left(\Phi_{\mathbf{i}, x}\right) w[\mathbf{i}]
$$

8.2. Denote by $\amalg$ the multiplication on $\pi^{*}\left(\mathcal{M}^{*}\right)$ obtained by pushing $\cdot$ with $\pi^{*}$, that is, for $\sigma, \tau \in \mathcal{M}^{*}$ set

$$
\pi^{*}(\sigma) \amalg \pi^{*}(\tau)=\pi^{*}(\sigma \cdot \tau) .
$$

LEMMA 8.1. - The product $\amalg$ is the restriction to $\pi^{*}\left(\mathcal{M}^{*}\right)$ of the classical shuffle product on $F^{*}$ defined by

$$
w\left[i_{1}, \ldots, i_{m}\right] \amalg w\left[i_{m+1}, \ldots, i_{m+n}\right]=\sum_{s} w\left[i_{s(1)}, \ldots, i_{s(m+n)}\right],
$$

where the sum runs over the permutations $s \in \mathfrak{S}_{m+n}$ such that

$$
s(1)<\cdots<s(m) \text { and } s(m+1)<\cdots<s(m+n) .
$$

Proof. - This follows easily from Lemma 6.1 and the duality of $\cdot$ and $\Delta$. Indeed,

$$
\begin{aligned}
\pi^{*}(\sigma \cdot \tau) & =\sum_{\mathbf{i}}(\sigma \cdot \tau)\left(d_{\mathbf{i}}\right) w[\mathbf{i}] \\
& =\sum_{\mathbf{i}}(\sigma \otimes \tau)\left(\Delta\left(d_{\mathbf{i}}\right)\right) w[\mathbf{i}] \\
& =\sum_{\mathbf{i}} \sum_{\left(\mathbf{c}^{\prime}, \mathbf{c}^{\prime \prime}\right)}\left(\sigma\left(d_{\mathbf{i}, \mathbf{c}^{\prime}}\right) \otimes \tau\left(d_{\mathbf{i}, \mathbf{c}^{\prime \prime}}\right)\right) w[\mathbf{i}]
\end{aligned}
$$

$4^{\mathrm{e}}$ SÉRIE - TOME $38-2005-\mathrm{N}^{\circ} 2$ 
where the pairs $\left(\mathbf{c}^{\prime}, \mathbf{c}^{\prime \prime}\right)$ are as in Lemma 6.1. Now it is clear that the coefficient of $w[\mathbf{i}]$ in this last sum is the same as the coefficient of $w[\mathbf{i}]$ in the shuffle product

$$
\left(\sum_{\mathbf{j}^{\prime}} \sigma\left(d_{\mathbf{j}^{\prime}}\right) w\left[\mathbf{j}^{\prime}\right]\right) \uplus\left(\sum_{\mathbf{j}^{\prime \prime}} \tau\left(d_{\mathbf{j}^{\prime \prime}}\right) w\left[\mathbf{j}^{\prime \prime}\right]\right)
$$

and the lemma is proved.

8.3. Suppose that $\mathfrak{g}$ is of type $\mathbb{A}_{n}$. Then $\mathcal{M}^{*} \simeq U(\mathfrak{n})^{*} \simeq \mathbb{C}[N]$, where $N$ is the group of unitriangular $(n+1) \times(n+1)$-matrices.

8.3.1. Let us construct an explicit isomorphism

$$
\alpha: \mathbb{C}[N] \rightarrow \mathcal{M}^{*} .
$$

Let $t_{i j}$ denote the coordinate function assigning to $n \in N$ its entry $n_{i j}$. Then

$$
\mathbb{C}[N]=\mathbb{C}\left[t_{i j} \mid 1 \leqslant i<j \leqslant n+1\right] .
$$

It is known that in the isomorphism $\mathbb{C}[N] \simeq U(\mathfrak{n})^{*}$, the natural basis of $\mathbb{C}[N]$ consisting of monomials in the $t_{i j}$ gets identified to the dual of the PBW-basis of $U(\mathfrak{n})$ associated to the quiver

$$
Q_{n}: \quad 1 \stackrel{\alpha_{1}}{\longleftarrow} 2 \longleftarrow \alpha_{2} \ldots \stackrel{\alpha_{n-1}}{\longleftarrow} n
$$

(see for example $[33,3.5])$. The $G_{\mathbf{V}}$-orbits of $\operatorname{rep}\left(Q_{n}, \mathbf{V}\right)$ are naturally labelled by the multisegments of degree $|\mathbf{V}|$, and if we denote by $\left\{\kappa_{\mathfrak{m}}^{*}\right\}$ the dual in $\mathcal{M}^{*}$ of the PBW-basis $\left\{\kappa_{\mathfrak{m}}\right\}$ in $\mathcal{M}$, then more precisely the above isomorphism maps the monomial $t_{i_{1} j_{1}} \cdots t_{i_{r} j_{r}}$ to the element $\kappa_{\mathrm{m}}^{*}$ indexed by the multisegment

$$
\mathfrak{m}=\left[i_{1}, j_{1}-1\right]+\cdots+\left[i_{r}, j_{r}-1\right] .
$$

For $i \leqslant j$, let $x[i, j]$ denote an indecomposable representation of $Q_{n}$ with socle $S_{i}$ and top $S_{j}$ (up to isomorphism there is exactly one such representation). Then the orbit of $x[i, j]$ is open dense so $\delta_{x[i, j]}$ belongs to $\mathcal{M}^{*}$ by Lemma 7.2. On the other hand, by Lemma 5.2

$$
\delta_{x[i, j]}\left(\kappa_{\mathfrak{m}}\right)= \begin{cases}1 & \text { if } \mathfrak{m}=[i, j] \\ 0 & \text { otherwise. }\end{cases}
$$

Hence $\kappa_{[i, j]}^{*}=\delta_{x[i, j]}$ and $\alpha$ is the algebra homomorphism determined by $\alpha\left(t_{i, j+1}\right)=\delta_{x[i, j]}$.

8.3.2. If we regard the functions $t_{i j}$ as entries of a unitriangular $(n+1) \times(n+1)$ matrix $T$ we may consider some special elements of $\mathbb{C}[N]$ given by the minors of this matrix.

PROPOSITION 8.2. - The images under $\alpha$ of all nonzero minors of the matrix $T$ belong to $\mathcal{S}^{*}$.

Proof. - We shall use the embedding $\pi^{*}$. First note that since $x[i, j]$ has a unique composition series,

$$
\pi^{*} \alpha\left(t_{i, j+1}\right)=w[j, j-1, \ldots, i] .
$$

Let $\varphi_{\underline{i} j}$ be the $k \times k$-minor taken on the sequence of rows $\underline{i}=\left(i_{1}<\cdots<i_{k}\right)$ and the sequence of columns $\underline{j}=\left(j_{1}<\cdots<j_{k}\right)$. Since $T$ is a unitriangular matrix with algebraically independent 
entries $t_{i j}$ above the diagonal, the function $\varphi_{\underline{i}}$ is nonzero if and only if $i_{r} \leqslant j_{r}$ for every $r$. We shall assume that this condition is satisfied. Let

$$
\lambda=\left(j_{k}, j_{k-1}+1, \ldots, j_{1}+k-1\right), \quad \mu=\left(i_{k}, i_{k-1}+1, \ldots, i_{1}+k-1\right) .
$$

Then $\lambda / \mu$ is a skew Young diagram. We identify it with the following subset of $\mathbb{Z}^{+} \times \mathbb{Z}^{+}$

$$
\lambda / \mu=\left\{(a, b) \mid 1 \leqslant b \leqslant k, \mu_{b}<a \leqslant \lambda_{b}\right\} .
$$

Each pair $(a, b)$ is called a cell of $\lambda / \mu$. Let $y$ be a standard Young tableau of shape $\lambda / \mu$, that is, a total ordering $c_{1}<\cdots<c_{t}$ of the cells of $\lambda / \mu$ which is increasing both on the rows and on the columns. We associate to $y$ the element

$$
w[y]=w\left[a_{t}-b_{t}, \ldots, a_{1}-b_{1}\right]
$$

of $F^{*}$, where $c_{r}=\left(a_{r}, b_{r}\right)(1 \leqslant r \leqslant t)$. Before we continue with the proof of Proposition 8.2 we need the following lemma.

LEMMA 8.3.-

$$
\pi^{*} \alpha\left(\varphi_{\underline{i}} \underline{j}\right)=\sum_{y} w[y]
$$

where $y$ runs over the set of standard Young tableaux of shape $\lambda / \mu$.

Proof. - Set

$$
D_{\underline{i} \underline{j}}=\pi^{*} \alpha\left(\varphi_{\underline{i}} \underline{j}\right) \quad \text { and } \quad S_{\lambda \mu}=\sum_{y} w[y] .
$$

We shall prove that $D_{\underline{i}} \underline{j}=S_{\lambda \mu}$ by induction on the number $t$ of cells of $\lambda / \mu$. If $t=1$, then $D_{\underline{i} \underline{j}}=w[i]=S_{\lambda \mu}$ for some $i$, and the statement is clear. So suppose $t>1$.

For $i=1, \ldots, n$ define $E_{i} \in \operatorname{End}_{\mathbb{C}}\left(F^{*}\right)$ by

$$
E_{i}\left(w\left[i_{1}, \ldots, i_{s}\right]\right)= \begin{cases}w\left[i_{1}, \ldots, i_{s-1}\right] & \text { if } i_{1}=i \\ 0 & \text { otherwise }\end{cases}
$$

It is immediate to check that $E_{i}$ is a derivation with respect to the shuffle product, i.e.

$$
E_{i}(f \amalg g)=E_{i}(f) \amalg g+f \amalg E_{i}(g) \quad\left(f, g \in F^{*}\right) .
$$

It is also clear that $f=g$ if and only if $E_{i}(f)=E_{i}(g)$ for every $i$. Note that $D_{\underline{i}} \underline{j}$ is the minor on rows $\underline{i}$ and columns $\underline{j}$ of the matrix

$$
W=\left(\begin{array}{ccccc}
1 & w[1] & w[2,1] & \ldots & w[n, n-1, \ldots, 1] \\
0 & 1 & w[2] & \ldots & w[n, n-1, \ldots, 2] \\
\vdots & \vdots & \vdots & & \vdots \\
0 & 0 & 0 & \ldots & 1
\end{array}\right)
$$

where in the expansion of the determinant the shuffle product of the entries is used. It follows that, if $j+1 \in \underline{j}$ and $j \notin \underline{j}$ then $E_{j}\left(D_{\underline{i} \underline{j}}\right)=D_{\underline{i k}}$ where $\underline{k}$ is obtained from $\underline{j}$ by replacing $j+1$ by $j$, and otherwise $E_{j}\left(\underline{D_{\underline{i}}}\right)=0$.

$4^{\mathrm{e}}$ SÉRIE - TOME $38-2005-\mathrm{N}^{\circ} 2$ 
On the other hand,

$$
E_{j}\left(S_{\lambda \mu}\right)=\sum_{z} w[z]
$$

where $z$ ranges over the Young tableaux whose shape is a skew Young diagram $\nu / \mu$ obtained from $\lambda / \mu$ by removing an outer cell $c=(a, b)$ with $a-b=j$. It is easy to check that there is one such diagram only if $j+1 \in \underline{j}$ and $j \notin \underline{j}$, and that this diagram then corresponds to the pair $(\underline{i}, \underline{k})$ above. So, by induction

$$
E_{j}\left(S_{\lambda \mu}\right)=S_{\nu \mu}=D_{\underline{i k}}=E_{j}\left(D_{\underline{i}} \underline{j}\right)
$$

in this case, and $E_{j}\left(S_{\lambda \mu}\right)=0=E_{j}\left(D_{\underline{i} j}\right)$, otherwise. Therefore $S_{\lambda \mu}=D_{\underline{i} j}$. This finishes the proof of Lemma 8.3.

We continue with the proof of Proposition 8.2. Let

$$
\mathfrak{m}=\left[i_{1}, j_{1}-1\right]+\cdots+\left[i_{k}, j_{k}-1\right]
$$

be the multisegment corresponding to the pair $(\underline{i}, \underline{j})$. (Here we leave out $\left[i_{l}, j_{l}-1\right]$ in case $i_{l}=j_{l}$.) Following [47] this parametrizes a laminated $\Lambda$-module $x[\mathfrak{m}]$, that is, a direct sum of indecomposable subquotients of projective $\Lambda$-modules. Let $\mathbf{V}$ be the underlying $I$-graded vector space of $x[\mathfrak{m}]$. It is known that the $G_{\mathbf{V}}$-orbit of $x[\mathfrak{m}]$ is open dense in its irreducible component, hence the function $\delta_{x[\mathfrak{m}]}$ belongs to the dual semicanonical basis.

Now it is easy to see that the types $\mathbf{i}$ of composition series of $x[\mathfrak{m}]$ are in one-to-one correspondence with the standard Young tableaux of shape $\lambda / \mu$, and that for each tableau, the corresponding flag variety $\Phi_{\mathbf{i}, x[\mathfrak{m}]}$ is reduced to a point. Therefore, comparing with Lemma 8.3 we see that

$$
\pi^{*}\left(\delta_{x[\mathfrak{m}]}\right)=\pi^{*} \alpha\left(\varphi_{\underline{i}} \underline{j}\right) .
$$

Hence $\alpha\left(\varphi_{\underline{i} j}\right)=\delta_{x[\mathfrak{m}]}$ belongs to the dual semicanonical basis. This finishes the proof of Proposition 8.2.

\section{A Galois covering of $\Lambda$ for type $\mathbb{A}_{n}$}

In order to prove the "if" part of Theorem 1.2 we need to study the canonical decomposition of $Z \in \operatorname{Irr}(\Lambda)$ for type $\mathbb{A}_{n}(n \leqslant 4)$. Our main tool for this will be the Auslander-Reiten quiver of $\Lambda$, which we will calculate by using a Galois covering $\widetilde{\Lambda}$ of $\Lambda$. This covering is in fact important for all $n$, and it will also play an essential rôle in our investigation of type $\mathbb{A}_{5}$ in the last sections of the paper. So we shall work in type $\mathbb{A}_{n}$ for general $n$ in the next two sections, and we shall specify which results are only valid for $n \leqslant 5$. We will also exclude the trivial case $\mathbb{A}_{1}$ and assume that $n \geqslant 2$.

9.1. For $n \geqslant 2$, let again $Q_{n}$ be the quiver

$$
1 \ll \stackrel{\alpha_{1}}{\longleftarrow} 2 \lessdot \alpha_{2}^{\alpha_{2}} \cdots \stackrel{\alpha_{n-1}}{\longleftarrow} n
$$

ANNALES SCIENTIFIQUES DE L'ÉCOLE NORMALE SUPÉRIEURE 


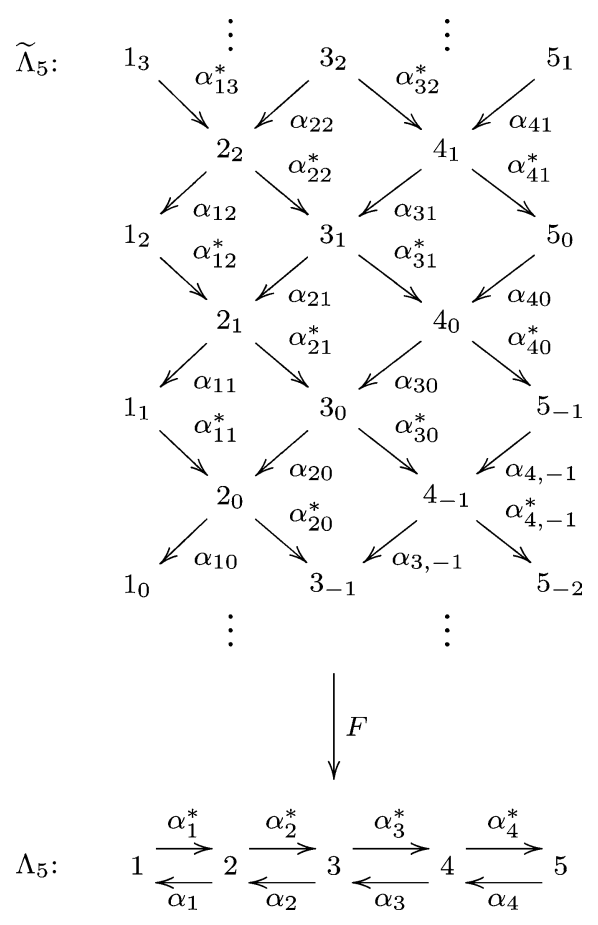

Fig. 1. The Galois covering.

of Dynkin type $\mathbb{A}_{n}$. Let $\Lambda_{n}=P\left(Q_{n}\right)$ be the preprojective algebra corresponding to $Q_{n}$. Thus $\Lambda_{n}=\mathbb{C} \overline{Q_{n}} / J_{n}$ where the double quiver $\overline{Q_{n}}$ of $Q_{n}$ is

$$
1 \stackrel{\alpha_{1}^{*}}{\underset{\alpha_{1}}{\longrightarrow}} 2 \underset{\alpha_{2}}{\stackrel{\alpha_{2}^{*}}{\rightleftarrows}} \ldots \stackrel{\alpha_{n-1}^{*}}{\underset{\alpha_{n-1}}{\longrightarrow}} n,
$$

and the ideal $J_{n}$ is generated by

$$
\alpha_{1} \alpha_{1}^{*}, \quad \alpha_{n-1}^{*} \alpha_{n-1}, \quad \alpha_{i}^{*} \alpha_{i}-\alpha_{i+1} \alpha_{i+1}^{*} \quad(1 \leqslant i \leqslant n-2) .
$$

9.2. Next, let $\widetilde{\Lambda}_{n}=\mathbb{C} \widetilde{Q}_{n} / \widetilde{J}_{n}$ where $\widetilde{Q}_{n}$ is the quiver with vertices $\left\{i_{j} \mid 1 \leqslant i \leqslant n, j \in \mathbb{Z}\right\}$ and arrows

$$
\alpha_{i j}:(i+1)_{j} \rightarrow i_{j}, \quad \alpha_{i j}^{*}: i_{j} \rightarrow(i+1)_{j-1} \quad(1 \leqslant i \leqslant n-1, j \in \mathbb{Z}),
$$

and the ideal $\widetilde{J}_{n}$ is generated by

$$
\alpha_{1 j} \alpha_{1, j+1}^{*}, \quad \alpha_{n-1, j}^{*} \alpha_{n-1, j}, \quad \alpha_{i j}^{*} \alpha_{i j}-\alpha_{i+1, j-1} \alpha_{i+1, j}^{*} \quad(1 \leqslant i \leqslant n-2, j \in \mathbb{Z}) .
$$

For $n=5$ we illustrate these definitions in Fig. 1 .

Denote by $\widetilde{Q}_{n}^{[a, b]}$ the full (and convex) subquiver of $\widetilde{Q}_{n}$ which has as vertices the set

$$
\left\{i_{j} \in \widetilde{Q}_{n} \mid a+3 \leqslant i+2 j \leqslant b+5\right\},
$$

$4^{\mathrm{e}}$ SÉRIE - TOME $38-2005-\mathrm{N}^{\circ} 2$ 
and denote by $\Delta_{n}^{[a, b]}$ the restriction of $\widetilde{\Lambda}_{n}$ to $\widetilde{Q}_{n}^{[a, b]}$, see 9.10 for an example.

9.3. The group $\mathbb{Z}$ acts on $\widetilde{\Lambda}_{n}$ by $\mathbb{C}$-linear automorphisms via

$$
z \cdot i_{j}=i_{j+z}, \quad z \cdot \alpha_{i j}=\alpha_{i, j+z}, \quad z \cdot \alpha_{i j}^{*}=\alpha_{i, j+z}^{*} .
$$

This induces an action

$$
\begin{gathered}
\mathbb{Z} \times \bmod \left(\widetilde{\Lambda}_{n}\right) \longrightarrow \bmod \left(\widetilde{\Lambda}_{n}\right) \\
(z, M) \mapsto{ }^{(z)} M,
\end{gathered}
$$

where ${ }^{(z)} M$ denotes the $\widetilde{\Lambda}_{n}$-module obtained from $M$ by twisting the action with $z$. Roughly speaking, ${ }^{(1)} M$ is the same $\widetilde{\Lambda}_{n}$-module as $M$, but "translated one level upwards".

If we consider $\widetilde{\Lambda}_{n}$ and $\Lambda_{n}$ as locally bounded categories we have a functor $F: \widetilde{\Lambda}_{n} \longrightarrow \Lambda_{n}$ defined by

$$
i_{j} \mapsto i, \quad \alpha_{i j} \mapsto \alpha_{i}, \quad \alpha_{i j}^{*} \mapsto \alpha_{i}^{*} .
$$

This is a Galois covering of $\Lambda_{n}$ in the sense of [22, §3.1], with Galois group $\mathbb{Z}$. It provides us with the push-down functor $[22, \S 3.2]$

$$
\bmod \left(\widetilde{\Lambda}_{n}\right) \longrightarrow \bmod \left(\Lambda_{n}\right)
$$

which we also denote by $F$. It is defined as follows. Let $x \in \bmod \left(\widetilde{\Lambda}_{n}, \mathbf{V}\right)$ be a $\widetilde{\Lambda}_{n}$-module with underlying graded vector space $\mathbf{V}=\bigoplus_{i, j} V_{i_{j}}$. Then $F(x)$ has the same underlying vector space with the grading $\mathbf{V}=\bigoplus_{i} V_{i}$ where $V_{i}=\bigoplus_{j} V_{i_{j}}$, and $F(x)$ has maps $F(x)_{\alpha_{i}}=\bigoplus_{j} x_{\alpha_{i j}}$ and $F(x)_{\alpha_{i}^{*}}=\bigoplus_{j} x_{\alpha_{i j}^{*}}$.

The next lemma follows from [22, §3.6] for $n \leqslant 4$, and from [16] for $n=5$, as noted in [15, $\S 6.3]$.

LEMma 9.1. - Let $2 \leqslant n \leqslant 5$. Any finite-dimensional indecomposable $\Lambda_{n}$-module is isomorphic to $F(x)$ for some indecomposable $\widetilde{\Lambda}_{n}$-module $x$, which is unique up to a translation $x \mapsto{ }^{(z)} x$ by the Galois group $\mathbb{Z}$.

9.4. For a dimension vector $\mathbf{d}=\left(d_{i_{j}}\right)_{1 \leqslant i \leqslant n, j \in \mathbb{Z}}$ for $\widetilde{\Lambda}_{n}$ define $d=\left(d_{1}, \ldots, d_{n}\right)$ where

$$
d_{i}=\sum_{j \in \mathbb{Z}} d_{i_{j}} .
$$

We have a morphism of varieties

$$
\mathrm{GL}(d) \times \bmod \left(\widetilde{\Lambda}_{n}, \mathbf{d}\right) \stackrel{F_{\mathbf{d}}}{\longrightarrow} \bmod \left(\Lambda_{n}, d\right)
$$

where the push-down morphism $F_{\mathbf{d}}$ is defined by $F_{\mathbf{d}}(g, M)=g \cdot F(M)$.

For $z \in \mathbb{Z}$ let $\mathbf{e}={ }^{(z)} \mathbf{d}$ be the $z$ th shift of $\mathbf{d}$, that is,

$$
e_{i_{j}}=d_{i_{j-z}} \quad(1 \leqslant i \leqslant n, j \in \mathbb{Z}) .
$$

Thus, if $\mathbf{d}$ is the dimension vector of a $\widetilde{\Lambda}_{n}$-module $M$, then $\mathbf{e}$ is the dimension vector of the shifted module ${ }^{(z)} M$. In this case we write $\mathbf{d} \sim \mathbf{e}$. This defines an equivalence relation $\sim$. 
To simplify our notation, we shall not always distinguish between $\mathbf{d}$ and its equivalence class. However, it will always be clear from the context which one is meant. To display a dimension vector (or its equivalence class) for $\widetilde{\Lambda}_{n}$ we just write down the relevant entries, all other entries are assumed to be 0 .

9.5. Recall that to any finite-dimensional algebra $A$ over a field $k$ (and more generally to any locally bounded $k$-algebra or locally bounded category, see e.g. [48, p. 54]) is associated a translation quiver $\Gamma_{A}$ called the Auslander-Reiten quiver of $A$. It contains a lot of information about the category $\bmod (A)$. In particular if $A$ is representation-finite and standard, one can recover $\bmod (A)$ from $\Gamma_{A}$.

The vertices of $\Gamma_{A}$ consist of the isomorphism classes of indecomposable finite-dimensional $A$-modules. If $M$ and $N$ are two such modules, then there $\operatorname{are}^{\operatorname{dim}_{k}} \mathcal{R}(M, N) / \mathcal{R}^{2}(M, N)$ arrows from $M$ to $N$ in $\Gamma_{A}$, where $\mathcal{R}$ denotes the radical of the category $\bmod (A)(\operatorname{compare}$ [45, Chapter 2]). This means that there is an arrow from $M$ to $N$ if there exists a nonzero irreducible homomorphism from $M$ to $N$. Here, by abuse of notation, we do not distinguish between a module and its isomorphism class. The quiver $\Gamma_{A}$ is endowed with an injective map $\tau$, the translation, defined on the subset of vertices corresponding to non-projective modules. If $M$ is indecomposable and non-projective then $\tau M=N$ where $0 \rightarrow N \rightarrow E \rightarrow M \rightarrow 0$ is the Auslander-Reiten sequence (or almost split sequence) ending in $M$.

The stable Auslander-Reiten quiver $\underline{\Gamma}_{A}$ of $A$ is obtained from $\Gamma_{A}$ by removing all translates $\tau^{-n} P$ and $\tau^{n} I(n \in \mathbb{N})$ of the projective vertices $P$ and the injective vertices $I$ as well as the arrows involving these vertices. Thus the translation $\tau$ induces a permutation on the vertices of $\underline{\Gamma}_{A}$.

We refer the reader to [1, Chapter VII] for more details on Auslander-Reiten theory, or to [21] and [45, Chapter 2] for $k$ algebraically closed as we assume here. Note however that these papers use slightly different conventions.

9.6. In Section 20.1 we display the Auslander-Reiten quivers of $\Lambda_{2}, \Lambda_{3}$ and $\Lambda_{4}$. To calculate them one first determines the Auslander-Reiten quivers of their coverings $\widetilde{\Lambda}_{2}, \widetilde{\Lambda}_{3}$ and $\widetilde{\Lambda}_{4}$. Indeed the algebras $\widetilde{\Lambda}_{n}$ are directed, that is, there is no sequence of indecomposable $\widetilde{\Lambda}_{n}$-modules of the form

$$
M \rightarrow M_{1} \rightarrow \cdots \rightarrow M_{t} \rightarrow M
$$

with all homomorphisms being nonzero and non-invertible. It follows that the Auslander-Reiten quiver of $\widetilde{\Lambda}_{n}$ can be calculated by a combinatorial procedure known as the knitting procedure, see for example [21, §6.6]. By applying the push-down functor $F$ to this quiver, one then obtains the Auslander-Reiten quiver of $\Lambda_{n}[22, \S 3.6]$.

In our pictures, each indecomposable $\Lambda_{n}$-module $M$ is represented by the dimension vector $\mathbf{d}$ of a $\widetilde{\Lambda}_{n}$-module $M_{\mathbf{d}}$ such that $F\left(M_{\mathbf{d}}\right)=M$. One has to identify each vertex in the extreme left column with the vertex in the extreme right column represented by the same dimension vector up to a shift by the Galois group $\mathbb{Z}$. The Auslander-Reiten quiver of $\Lambda_{3}$ is shaped like a Moebius band, and for $\Lambda_{2}$ and $\Lambda_{4}$ one gets a cylinder.

In particular, we see that $\Lambda_{2}$ has 4 isoclasses of indecomposable modules, $\Lambda_{3}$ has 12 , and $\Lambda_{4}$ has 40 .

We should point out that for $n>5$ there are $\Lambda_{n}$-modules which are not obtained from a $\widetilde{\Lambda}_{n}$-module via the push-down functor.

9.7. For an algebra $A$ let $\bmod (A)$ be the stable category of finite-dimensional $A$-modules [48, p. 55]. By definition, the objects of $\underline{\bmod }(A)$ are the same as the objects of $\bmod (A)$, and the

$4^{\mathrm{e}}$ SÉRIE - TOME $38-2005-\mathrm{N}^{\circ} 2$ 
morphism space $\operatorname{Hom}_{A}(M, N)$ is defined as $\operatorname{Hom}_{A}(M, N)$ modulo the morphisms factoring through projective modules. The isomorphism classes of indecomposable objects in $\bmod (A)$ correspond naturally to the isomorphism classes of non-projective indecomposable $A$-modules.

The stable category $\underline{\bmod }(A)$ is no longer abelian, but if $A$ is a Frobenius algebra then $\underline{\bmod }(A)$ has the structure of a triangulated category [26, §I.2] with translation functor $\Omega^{-1}$, the inverse of Heller's loop functor.

Moreover, in this situation we may identify the quiver of the stable category $\underline{\bmod }(A)$ (see for example [26, §I.4.8]) with the stable Auslander-Reiten quiver $\underline{\Gamma}_{A}$ defined in 9.5 - just remove the projective-injective vertices from $\Gamma_{A}$.

9.8. To a finite-dimensional algebra $B$ one associates its repetitive algebra $\widehat{B}$ ([26, p. 59], [48, p. 57]). This is an infinite-dimensional algebra without unit. However it is locally bounded, and its indecomposable projective modules coincide with its indecomposable injective modules, that is, $\widehat{B}$ is a Frobenius algebra.

Hence for a finite-dimensional algebra $B, \underline{\bmod }(\widehat{B})$ is a triangulated category, see 9.7. Moreover if $B$ has finite global dimension, there is an equivalence of triangulated categories between $\bmod (\widehat{B})$ and the derived category $D^{b}(\bmod (B))$ of bounded complexes of $B$-modules (Happel's Theorem [26, §II.4]). Under this equivalence the functor $\Omega^{-1}$ corresponds to the translation functor $M \mapsto M[1]$.

9.9. If $B$ is a finite-dimensional algebra of finite global dimension we have by [27, §3.2]

$$
K_{0}(\bmod (\widehat{B}))=K_{0}(\bmod (B)) \oplus \mathcal{P}(\widehat{B}),
$$

where $\mathcal{P}(\widehat{B})$ is the subgroup of $K_{0}(\bmod (\widehat{B}))$ generated by the classes of projective $\widehat{B}$-modules. Thus we may identify $K_{0}(\underline{\bmod }(\widehat{B}))$ with $K_{0}(\bmod (B))$. This coincides with the Grothendieck group of $\bmod (\widehat{B})$ viewed as a triangulated category.

For a $\widehat{B}$-module $M$ we denote by $[M]$ its class in $K_{0}(\bmod (B))=K_{0}(\underline{\bmod }(\widehat{B}))$. In particular, if $M$ is projective, then $[M]=0$. Notice that $[M]$ depends only on the dimension vector $\operatorname{dim}(M) \in K_{0}(\bmod (\widehat{B}))$ and (9) provides an efficient method for calculating $[M]$. In this context we have

$$
[\tau M]=\Phi[M]
$$

where $\Phi$ is the Coxeter transformation of $B$ and $\tau$ is the self-equivalence of $\bmod (\widehat{B})$ induced by the Auslander-Reiten translation of $\bmod (\widehat{B})$. This follows from Happel's Theorem 9.8, the construction of the Auslander-Reiten translate in $[26, \S$ I.4.6] and the definition of the Coxeter transformation.

9.10. In case $2 \leqslant n \leqslant 5$ we have in $\underline{\bmod }\left(\widetilde{\Lambda}_{n}\right)$ a functorial isomorphism

$$
{ }^{(1)} M \cong \Omega^{-1} \tau^{-1} M
$$

which is proved along the lines of $[15, \S 6.4]$. In particular this implies $\left[{ }^{(1)} M\right]=-\Phi^{-1}[M]$. 
Moreover, if we set $\Delta_{n}=\Delta_{n}^{[0, n-4]}$ it is easy to see that $\widetilde{\Lambda}_{n}=\widehat{\Delta}_{n}$. For example $\Delta_{5}$ is the algebra given by the following quiver with relations:

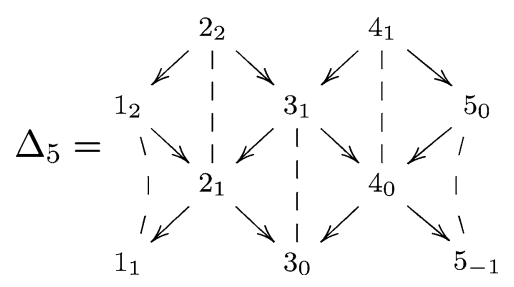

Here the dotted lines indicate zero relations and commutativity relations.

Thus by Happel's Theorem 9.8 we have $\bmod \left(\widetilde{\Lambda}_{n}\right) \cong D^{b}\left(\bmod \left(\Delta_{n}\right)\right)$. In particular we can apply 9.9. Note that $\Delta_{n}$ is hereditary of type $\mathbb{A}_{1}$ (resp. $\mathbb{A}_{3}$ ) for $n=2$ (resp. $n=3$ ), and tilted of type $\mathbb{D}_{6}$ in case $n=4$. Happel's description of the Auslander-Reiten quiver of the derived category of a (piecewise) hereditary algebra of Dynkin type [26, §I.5.6, §IV.4.3] together with (11) yields the shape of the stable Auslander-Reiten quiver in these cases. In case $n=5$ the algebra $\Delta_{5}$ is tubular and we can derive the structure of $\bmod \left(\widetilde{\Lambda}_{5}\right)$ from the known structure of the derived category of a tubular algebra [27]. We will discuss this case in Section 14.

\section{From Schur roots to indecomposable multisegments}

10.1. Let $I\left(\widetilde{\Lambda}_{n}\right)$ be the set of dimension vectors of the indecomposable $\widetilde{\Lambda}_{n}$-modules. By $I_{S}\left(\widetilde{\Lambda}_{n}\right)$ we denote the set of Schur roots of $\widetilde{\Lambda}_{n}$, i.e. the set of dimension vectors of the (indecomposable) $\widetilde{\Lambda}_{n}$-modules $M$ with $\operatorname{End}_{\widetilde{\Lambda}_{n}}(M) \cong \mathbb{C}$. For $\mathbf{d} \in I_{S}\left(\widetilde{\Lambda}_{n}\right)$ let

$$
Z_{\mathbf{d}}=\overline{\left\{M \in \bmod \left(\widetilde{\Lambda}_{n}, \mathbf{d}\right) \mid \operatorname{End}_{\widetilde{\Lambda}_{n}}(M) \cong \mathbb{C}\right\}} .
$$

For $2 \leqslant n \leqslant 5$ this is an irreducible component of $\bmod \left(\widetilde{\Lambda}_{n}, \mathbf{d}\right)$. We write $Z_{\mathbf{d}} \sim Z_{\mathbf{e}}$ if $\mathbf{d} \sim \mathbf{e}$.

For an irreducible component $Z$ of $\bmod \left(\widetilde{\Lambda}_{n}, \mathbf{d}\right)$ define

$$
\eta(Z)=\overline{F_{\mathbf{d}}(\mathrm{GL}(d) \times Z)} .
$$

Clearly, $\eta(Z)$ is an irreducible subset of $\bmod \left(\Lambda_{n}, d\right)$, but in general $\eta(Z)$ is not an irreducible component. The following result was shown in [24].

THEOREM 10.1. - Assume that $2 \leqslant n \leqslant 5$. For $\mathbf{d} \in I_{S}\left(\widetilde{\Lambda}_{n}\right)$, the variety $\eta\left(Z_{\mathbf{d}}\right)$ is an indecomposable irreducible component of $\bmod \left(\Lambda_{n}, d\right)$. Moreover, $\eta$ induces a bijection from $I_{S}\left(\widetilde{\Lambda}_{n}\right) / \sim$ to $\operatorname{ind}\left(\operatorname{Irr}\left(\Lambda_{n}\right)\right)$.

10.2. Let $\mathbf{M}(n)$ be the set of multisegments supported on $\{1, \ldots, n\}$, that is, of the form

$$
\mathfrak{m}=\sum_{1 \leqslant i \leqslant j \leqslant n} m_{i j}[i, j]
$$

where $m_{i j} \in \mathbb{N}$. The degree $d=\left(d_{1}, \ldots, d_{n}\right)$ of $\mathfrak{m}$ is given by

$$
d_{k}=\sum_{i \leqslant k \leqslant j} m_{i j} \quad(1 \leqslant k \leqslant n) .
$$

$4^{\text {e }}$ SÉRIE - TOME $38-2005-\mathrm{N}^{\circ} 2$ 
There is a one-to-one correspondence $\mathfrak{m} \mapsto \mathcal{O}_{\mathfrak{m}}$ between the set $\mathbf{M}_{d}(n)$ of multisegments of degree $d$ in $\mathbf{M}(n)$ and the set of $\mathrm{GL}(d)$-orbits in $\bmod \left(\mathbb{C} Q_{n}, d\right)$.

Let $\mathfrak{m}_{\max }(d)$ be the multisegment labelling the unique dense orbit of $\bmod \left(\mathbb{C} Q_{n}, d\right)$. It can be computed recursively by

$$
\mathfrak{m}_{\max }(d)=[a, b]+\mathfrak{m}_{\max }(d-e)
$$

where

$$
a=\min \left\{i \mid d_{i} \neq 0\right\} \quad \text { and } \quad b=\max \left\{j \mid d_{i} \neq 0 \text { for all } a \leqslant i \leqslant j\right\},
$$

and $e_{i}=1$ if $i \in[a, b]$ and $e_{i}=0$, otherwise. For example,

$$
\mathfrak{m}_{\max }(1,2,3,1,2)=[1,5]+[2,3]+[3,3]+[5,5] .
$$

10.3. Let $\pi_{d}$ be the projection morphism from $\bmod \left(\Lambda_{n}, d\right)$ to $\bmod \left(\mathbb{C} Q_{n}, d\right)$ obtained by forgetting the maps labelled by the arrows $\alpha_{i}^{*}$. Lusztig [38] proved that the irreducible components of $\bmod \left(\Lambda_{n}, d\right)$ are the closures of the sets $\pi_{d}^{-1}\left(\mathcal{O}_{\mathfrak{m}}\right), \mathfrak{m} \in \mathbf{M}_{d}(n)$. Define

$$
Z_{\mathfrak{m}}=\overline{\pi_{d}^{-1}\left(\mathcal{O}_{\mathfrak{m}}\right)}
$$

A multisegment $\mathfrak{m}$ is said to be indecomposable if $Z_{\mathfrak{m}}$ is indecomposable. By ind $(\mathbf{M}(n))$ we denote the set of indecomposable multisegments in $\mathbf{M}(n)$.

10.4. For a dimension vector $\mathbf{d}=\left(d_{i_{j}}\right)_{1 \leqslant i \leqslant n, j \in \mathbb{Z}}$ for $\widetilde{\Lambda}_{n}$ set

$$
\mathfrak{m}_{\max }(\mathbf{d})=\sum_{j \in \mathbb{Z}} \mathfrak{m}_{\max }(d(j))
$$

where $d(j)=\left(d_{1_{j}}, d_{2_{j}}, \ldots, d_{n_{j}}\right)$. The variety $\bmod \left(\widetilde{\Lambda}_{n}, d(j)\right)$ is irreducible and, in fact, isomorphic to $\bmod \left(\mathbb{C} Q_{n}, d(j)\right)$. There is a $\widetilde{\Lambda}_{n}$-module $M_{d(j)}$ such that the orbit $\mathcal{O}\left(M_{d(j)}\right)$ is dense in $\bmod \left(\widetilde{\Lambda}_{n}, d(j)\right)$, and $M_{d(j)}$ is uniquely determined up to isomorphism.

Let $\mathcal{F}(\mathbf{d})$ be the set of modules $M \in \bmod \left(\widetilde{\Lambda}_{n}, \mathbf{d}\right)$ which have submodules $\left(M_{j}\right)_{j \in \mathbb{Z}}$ such that $M_{j-1} \subseteq M_{j}$ and $M_{j} / M_{j-1} \cong M_{d(j)}$ for all $j \in \mathbb{Z}$. Thus $M_{j}=0$ if and only if $d(i)=0$ for all $i \leqslant j$, and $M_{j-1}=M_{j}$ if and only if $d(j)=0$. Set

$$
Z_{\max }(\mathbf{d})=\overline{\mathcal{F}(\mathbf{d})}
$$

For $Z \in \operatorname{Irr}\left(\widetilde{\Lambda}_{n}\right)$ let $\mu(Z)$ be the unique multisegment in $\mathbf{M}(n)$ such that

$$
\overline{\mathcal{O}_{\mu(Z)}}=\pi_{d}(\eta(Z)) \text {. }
$$

This defines a map

$$
\mu: \operatorname{Irr}\left(\widetilde{\Lambda}_{n}\right) \longrightarrow \mathbf{M}(n) .
$$

If $\eta(Z)$ is an irreducible component, say $Z_{\mathfrak{m}}$, of $\bmod \left(\Lambda_{n}, d\right)$, then $\mu(Z)=\mathfrak{m}$.

LEMMA 10.2. - For all dimension vectors $\mathbf{d}$ for $\widetilde{\Lambda}_{n}$ the set $Z_{\max }(\mathbf{d})$ is an irreducible component of $\bmod \left(\widetilde{\Lambda}_{n}, \mathbf{d}\right)$, and we have

$$
\mu\left(Z_{\max }(\mathbf{d})\right)=\mathfrak{m}_{\max }(\mathbf{d}) .
$$


Proof. - One can easily see that

$$
\operatorname{Ext}_{\widetilde{\Lambda}_{n}}^{1}\left(M_{d(i)}, M_{d(j)}\right)=0 \quad \text { for all } i \leqslant j .
$$

Then [14, Theorem 1.3] yields that $Z_{\max }(\mathbf{d})$ is an irreducible component. The second part of the lemma follows directly from the definitions.

10.5. Assume that $2 \leqslant n \leqslant 5$. Define $\psi: I_{S}\left(\widetilde{\Lambda}_{n}\right) \rightarrow \mathbf{M}(n)$ by

$$
\psi(\mathbf{d})= \begin{cases}2[1,1]+[2,2]+[2,4]+[3,3]+[4,5] & \text { if } n=5 \text { and } \mathbf{d}=\left(\begin{array}{ccc}
1 & 0 & 0 \\
1 & 1 & 1 \\
1 & 2 & 1 \\
1 & 1
\end{array}\right) \\
{[1,2]+[2,4]+[3,3]+[4,4]+2[5,5]} & \text { if } n=5 \text { and } \mathbf{d}=\left(\begin{array}{ccc}
1 & 1 \\
1 & 2 & 1 \\
1 & 1 \\
0 & 0 & 1
\end{array}\right) \\
\mathfrak{m}_{\max }(\mathbf{d}) & \text { otherwise. }\end{cases}
$$

THEOREM 10.3. - Let $2 \leqslant n \leqslant 5$. The map $\psi$ establishes a bijection from $I_{S}\left(\widetilde{\Lambda}_{n}\right) / \sim$ to $\operatorname{ind}(\mathbf{M}(n))$. Moreover the following diagram commutes:

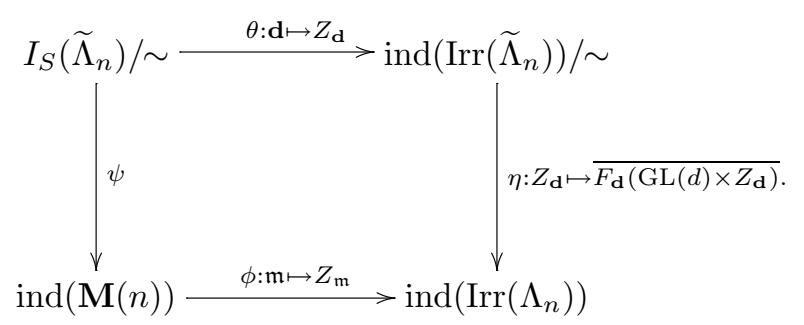

The proof of Theorem 10.3 will be given in Section 18.

\section{Cases $\mathbb{A}_{2}, \mathbb{A}_{3}, \mathbb{A}_{4}$ : the component graph}

11.1. In the case that $\Lambda_{n}$ is of finite representation type, the results of the previous sections simplify greatly.

THEOREM 11.1. - Assume that $n=2,3$, 4. Then for each $\mathbf{d} \in I\left(\widetilde{\Lambda}_{n}\right)$ there exists (up to isomorphism) exactly one indecomposable $\widetilde{\Lambda}_{n}$-module $M_{\mathbf{d}}$ with dimension vector $\mathbf{d}$. Furthermore, $\operatorname{End}_{\widetilde{\Lambda}_{n}}\left(M_{\mathbf{d}}\right) \cong \mathbb{C}$ for all d, i.e. $I_{S}\left(\widetilde{\Lambda}_{n}\right)=I\left(\widetilde{\Lambda}_{n}\right)$. Therefore,

$$
\operatorname{ind}\left(\operatorname{Irr}\left(\Lambda_{n}\right)\right)=\left\{\eta\left(Z_{\mathbf{d}}\right) \mid \mathbf{d} \in I\left(\widetilde{\Lambda}_{n}\right) / \sim\right\}
$$

and

$$
\operatorname{ind}(\mathbf{M}(n))=\left\{\mathfrak{m}_{\max }(\mathbf{d}) \mid \mathbf{d} \in I\left(\widetilde{\Lambda}_{n}\right) / \sim\right\} .
$$

Proof. - The first two statements follow from the general theory of directed (simply connected representation-finite) algebras [45, §2.4.(8)]. Then we apply Theorems 10.1 and 10.3.

$4^{\mathrm{e}}$ SÉRIE - TOME $38-2005-\mathrm{N}^{\circ} 2$ 
11.2. In Section 20.2 we list the 40 indecomposable multisegments in $\mathbf{M}(4)$ labelling the 40 indecomposable $\Lambda_{4}$-modules, and we redisplay the Auslander-Reiten quiver of $\Lambda_{4}$ with vertices these multisegments. The translation $\tau$ can be read off by going horizontally two units to the left, for example

$$
\tau\left(\mathfrak{m}_{1}\right)=\mathfrak{m}_{16}, \quad \tau\left(\mathfrak{m}_{2}\right)=\mathfrak{m}_{29}, \quad \tau\left(\mathfrak{m}_{3}\right)=\mathfrak{m}_{27},
$$

and so on. Note that $\mathfrak{m}_{37}, \mathfrak{m}_{38}, \mathfrak{m}_{39}, \mathfrak{m}_{40}$ have no $\tau$-translate because they are the projective vertices.

11.3. We shall use $\Gamma_{\Lambda_{n}}$ to describe the pairs $(X, Y)$ of indecomposable $\Lambda_{n}$-modules such that $\operatorname{Ext}_{\Lambda_{n}}^{1}(X, Y)=0$. By Proposition 3.2, $\operatorname{Ext}_{\Lambda_{n}}^{1}(X, Y)=0$ if and only if $\operatorname{Ext}_{\Lambda_{n}}^{1}(Y, X)=0$, so this is a symmetric relation.

Recall, that for any finite-dimensional algebra $\Lambda$ the Auslander-Reiten formula [1, Proposition 4.5] gives us

$$
D \operatorname{Ext}_{\Lambda}^{1}(X, Y) \cong \underline{\operatorname{Hom}}_{\Lambda}\left(\tau^{-1} Y, X\right) .
$$

Now, if $\Lambda$ is selfinjective, representation-finite and admits a simply connected Galois covering $\widetilde{\Lambda}$ with Galois group $G$, we get

$$
D \operatorname{Ext}_{\Lambda}^{1}(X, Y) \cong \underline{\operatorname{Hom}}_{\Lambda}\left(\tau^{-1} Y, X\right) \cong \bigoplus_{g \in G} \underline{\operatorname{Hom}}_{\widetilde{\Lambda}}\left({ }^{(g)}(\tau \tilde{Y}), \tilde{X}\right) .
$$

Here $X$ and $Y$ are indecomposable $\Lambda$-modules, and $\tilde{X}$ and $\tilde{Y}$ are indecomposable $\widetilde{\Lambda}$-modules which under push-down give $X$ and $Y$, respectively. In this situation it is easy to determine the dimensions of the summands in the last term using additive functions on the stable AuslanderReiten quiver $\underline{\Gamma}_{\widetilde{\Lambda}}=\mathbb{Z} \Theta$ for some Dynkin quiver $\Theta$, see $[21, \S 6.5]$.

As we have seen, this is exactly the situation for $\Lambda=\Lambda_{n}(n \leqslant 4)$, and by (11) there will be at most one $i \in \mathbb{Z}$ with $\underline{\operatorname{Hom}}_{\tilde{\Lambda}}\left({ }^{(i)}(\tau \tilde{Y}), \tilde{X}\right) \neq 0$.

Since $\tau$ induces a self-equivalence of $\underline{\bmod }(\Lambda)$ we have to do this calculation only for one representative $X$ of each $\tau$-orbit.

The stable Auslander-Reiten quivers of $\Lambda_{2}, \Lambda_{3}$ and $\Lambda_{4}$ have 1,2 and 6 non-trivial $\tau$-orbits, respectively.

For $n=2$ there are only two indecomposable non-projective $\Lambda_{n}$-modules, say $X$ and $Y$, and $\operatorname{Ext}_{\Lambda_{2}}^{1}(X, Y) \neq 0$.

In Sections 20.3 and 20.4 we display several copies of the stable Auslander-Reiten quivers of $\Lambda_{3}$ and $\Lambda_{4}$. In each copy we pointed a representative $X_{i}$ of a $\tau$-orbit, and we marked with the sign $\bullet$ all indecomposable $\Lambda_{n}$-modules $M$ such that

$$
\operatorname{Ext}_{\Lambda_{n}}^{1}\left(M, X_{i}\right) \neq 0 .
$$

For example, there are 21 indecomposable $\Lambda_{4}$-modules $M$ such that

$$
\operatorname{Ext}_{\Lambda_{4}}^{1}\left(M, X_{2}\right) \neq 0 .
$$

11.4. We note that the previous description shows in particular that for $n \leqslant 4$ every indecomposable $\Lambda_{n}$-module $X$ satisfies

$$
\operatorname{Ext}_{\Lambda_{n}}^{1}(X, X)=0 .
$$


This was first observed by Marsh and Reineke. It follows that the orbit closures of the indecomposable $\Lambda_{n}$-modules are the indecomposable irreducible components of the varieties of $\Lambda_{n}$-modules. Therefore the results of 11.3 give a complete description of the component graph $\mathcal{C}\left(\Lambda_{n}\right)$ for $n \leqslant 4$.

\section{Cases $\mathbb{A}_{2}, \mathbb{A}_{3}, \mathbb{A}_{4}$ : the graph of prime elements of $\mathcal{B}^{*}$}

In this section we consider the dual canonical basis $\mathcal{B}^{*}$ of $\mathbb{C}[N]$ for type $\mathbb{A}_{n}(n \leqslant 4)$. Using the bijection $\mathfrak{m} \mapsto Z_{\mathfrak{m}}$ we may label its elements by multisegments $\mathfrak{m}$ or irreducible components $Z$. We will write $\mathcal{B}^{*}=\left\{b_{\mathfrak{m}}^{*} \mid \mathfrak{m} \in \mathbf{M}(n)\right\}$ or $\mathcal{B}^{*}=\left\{b_{Z}^{*} \mid Z \in \operatorname{Irr}\left(\Lambda_{n}\right)\right\}$ depending on the context.

12.1. An element $b^{*} \in \mathcal{B}^{*}-\{1\}$ is called prime if it does not have a non-trivial factorization $b^{*}=b_{1}^{*} b_{2}^{*}$ with $b_{1}^{*}, b_{2}^{*} \in \mathcal{B}^{*}$. Let $\mathcal{P}_{n}$ be the set of prime elements in $\mathcal{B}^{*}$.

Let $\mathcal{G}_{n}$ be the graph of prime elements. The set of vertices of $\mathcal{G}_{n}$ is $\mathcal{P}_{n}$, and there is an edge between $b_{1}^{*}$ and $b_{2}^{*}$ if and only if the product $b_{1}^{*} b_{2}^{*}$ is in $\mathcal{B}^{*}$. These graphs give a complete description of the basis $\mathcal{B}^{*}$ [4]. Indeed, $\mathcal{B}^{*}$ is the collection of all monomials of the form

$$
\prod_{\mathfrak{m} \in \operatorname{ind}(\mathbf{M}(n))}\left(b_{\mathfrak{m}}^{*}\right)^{k(\mathfrak{m})}
$$

where the $k(\mathfrak{m}) \in \mathbb{N}$ satisfy for $\mathfrak{m} \neq \mathfrak{m}^{\prime}$

$$
\left(k(\mathfrak{m}) k\left(\mathfrak{m}^{\prime}\right) \neq 0\right) \quad \Longrightarrow \quad\left(\left(\mathfrak{m}, \mathfrak{m}^{\prime}\right) \in \mathcal{G}_{n}\right) .
$$

Note that for all $b^{*}$ in $\mathcal{B}^{*}$, the square of $b^{*}$ also belongs to $\mathcal{B}^{*}$. This is a particular case of the BZconjecture which holds for $\mathbb{A}_{n}(n \leqslant 4)$. Therefore there is a loop at each vertex of $\mathcal{G}_{n}$. Similarly, the $n$ vertices labelled by an irreducible component containing an indecomposable projective module are connected to every other vertex (because the corresponding elements of $\mathcal{B}_{q}^{*}$ belong to the $q$-center). Let $\mathcal{G}_{n}^{\circ}$ be the graph obtained from $\mathcal{G}_{n}$ by deleting these $n$ vertices and all the loops. Clearly $\mathcal{G}_{n}^{\circ}$ contains all the information.

12.2. The graphs $\mathcal{G}_{2}^{\circ}$ and $\mathcal{G}_{3}^{\circ}$ have been determined by Berenstein and Zelevinsky [4]. They are respectively dual to an associahedron of type $\mathbb{A}_{1}$ and $\mathbb{A}_{3}$ in the terminology of $[11,19,20]$. In 20.5 we display the graph $\mathcal{G}_{4}^{\circ}$, which has 36 vertices (corresponding to the multisegments $\mathfrak{m}_{1}, \ldots, \mathfrak{m}_{36}$ in the list of 20.2) and 330 edges. We have calculated it by computer using the BZconjecture. As suggested by Zelevinsky, $\mathcal{G}_{4}^{\circ}$ is dual to an associahedron of type $\mathbb{D}_{6}$. The maximal complete subgraphs of $\mathcal{G}_{n}^{\circ}$ all have the same cardinality, namely 1, 3 and 6 for $n=2,3$ and 4 , and are called clusters. There are respectively 2,14 and 672 clusters.

12.3. The following theorem was proved by Marsh and Reineke for $n \leqslant 3$ and conjectured for $n=4$ [42].

THEOREM 12.1. - For $n \leqslant 4$ the graph $\mathcal{G}_{n}$ is isomorphic to $\mathcal{C}\left(\Lambda_{n}\right)$ via the map $b_{\mathfrak{m}}^{*} \mapsto Z_{\mathfrak{m}}$.

Proof. - This is checked by using the explicit descriptions of both graphs. For example, from the first quiver of Section 20.4 we get that the vertex labelled by $\mathfrak{m}_{4}$ in $\mathcal{C}\left(\Lambda_{4}\right)$ is connected to all other vertices except

$$
\mathfrak{m}_{3}, \mathfrak{m}_{7}, \mathfrak{m}_{8}, \mathfrak{m}_{11}, \mathfrak{m}_{12}, \mathfrak{m}_{13}, \mathfrak{m}_{14}, \mathfrak{m}_{15}, \mathfrak{m}_{31}, \mathfrak{m}_{32} .
$$

The same happens in the graph $\mathcal{G}_{4}$, as can be seen from Section 20.5 .

$4^{\mathrm{e}}$ SÉRIE - TOME $38-2005-\mathrm{N}^{\circ} 2$ 


\section{End of the proof of Theorem 1.2}

In this section we prove the "if" part of Theorem 1.2.

13.1. Let $\mathfrak{g}$ be of type $\mathbb{A}_{4}$. For brevity set $\rho_{\mathfrak{m}}=\rho_{Z_{\mathfrak{m}}}$ for $\mathfrak{m} \in \mathbf{M}(4)$.

PROPOSITION 13.1. - For every indecomposable multisegment $\mathfrak{m}$ of $\mathbf{M}(4)$ we have

$$
b_{\mathfrak{m}}^{*}=\rho_{\mathfrak{m}} .
$$

Proof. - For 34 multisegments $\mathfrak{m}_{i}$ out of the 40 elements of $\operatorname{ind}(\mathbf{M}(4))$ the vector $b_{\mathfrak{m}_{i}}^{*}$ is a minor, and the result follows from Proposition 8.2.

The six elements which are not minors are $b_{\mathfrak{m}_{i}}^{*}$ where $31 \leqslant i \leqslant 36$. Denoting by $\zeta$ the multisegment duality of Zelevinsky [52], we have

$$
\zeta\left(\mathfrak{m}_{31}\right)=\mathfrak{m}_{32}, \quad \zeta\left(\mathfrak{m}_{33}\right)=\mathfrak{m}_{34}, \quad \zeta\left(\mathfrak{m}_{35}\right)=\mathfrak{m}_{36} .
$$

Denote also by $\zeta$ the linear involution on $F^{*}$ given by

$$
\zeta\left(w\left[i_{1}, \ldots, i_{k}\right]\right)=w\left[i_{k}, \ldots, i_{1}\right] .
$$

It follows from $[38,41]$ that

$$
\zeta\left(\pi^{*}\left(\mathcal{B}^{*}\right)\right)=\pi^{*}\left(\mathcal{B}^{*}\right) \text { and } \quad \zeta\left(\pi^{*}\left(\mathcal{S}^{*}\right)\right)=\pi^{*}\left(\mathcal{S}^{*}\right) .
$$

Moreover it is known [52] that for any multisegment $\mathfrak{m}$ we have

$$
\zeta\left(\pi^{*}\left(b_{\mathfrak{m}}^{*}\right)\right)=\pi^{*}\left(b_{\zeta(\mathfrak{m})}^{*}\right) .
$$

Hence it is enough to prove the lemma for $i=31,33,35$. This can be checked by an explicit computation in $F^{*}$. The calculation of $\pi^{*}\left(b_{\mathfrak{m}}^{*}\right)$ is easy to perform using the algorithm of [32]. On the other hand, for $x_{i}$ a point in the dense orbit of the irreducible component $Z_{\mathfrak{m}_{i}}$, we have $\rho_{\mathfrak{m}_{i}}=\delta_{x_{i}}$ and $\pi^{*}\left(\delta_{x_{i}}\right)$ can be computed via (8) in Section 8. Thus, for $i=31$ we obtain the following expression for both $\pi^{*}\left(b_{\mathfrak{m}_{i}}^{*}\right)$ and $\pi^{*}\left(\rho_{\mathfrak{m}_{i}}\right)$ :

$$
\begin{aligned}
& 2 w[4,2,3,3,1,2]+2 w[2,4,3,1,3,2]+2 w[2,4,3,3,1,2]+w[2,1,4,3,2,3] \\
& \quad+w[2,1,3,4,3,2]+2 w[2,1,4,3,3,2]+w[2,3,4,1,3,2]+w[4,2,1,3,2,3] \\
& \quad+w[2,3,4,3,1,2]+w[4,3,2,1,3,2]+w[4,3,2,3,1,2]+2 w[4,2,1,3,3,2] \\
& \quad+w[2,4,3,1,2,3]+w[2,4,1,3,2,3]+2 w[2,4,1,3,3,2]+w[2,3,1,4,3,2] \\
& \quad+2 w[4,2,3,1,3,2]+w[4,2,3,1,2,3] .
\end{aligned}
$$

The calculations for $i=33$ and $i=35$ are similar and we omit them.

13.2. We can now finish the proof of Theorem 1.2. Assume that $\mathfrak{g}$ is of type $\mathbb{A}_{4}$. Let

$$
Z=\overline{Z_{1} \oplus \cdots \oplus Z_{r}}
$$

be the canonical decomposition of an irreducible component $Z \in \operatorname{Irr}\left(\Lambda_{4}\right)$. By Corollary 7.5

$$
\rho_{Z}=\rho_{Z_{1}} \cdots \rho_{Z_{r}} .
$$

ANNALES SCIENTIFIQUES DE L'ÉCOLE NORMALE SUPÉRIEURE 
All components $Z_{k}, 1 \leqslant k \leqslant r$ are of the form

$$
Z_{k}=Z_{\mathfrak{m}_{i_{k}}}
$$

for some indecomposable multisegment $\mathfrak{m}_{i_{k}}$. By Proposition 13.1 we thus have $\rho_{Z_{k}}=b_{Z_{k}}^{*}$. Moreover, using Theorem 12.1 we get

$$
b_{Z_{1}}^{*} \cdots b_{Z_{r}}^{*} \in \mathcal{B}^{*}
$$

Hence $\rho_{Z}$ belongs to $\mathcal{B}^{*}$. Thus for Dynkin types $\mathbb{A}_{n}$ with $n \leqslant 4$ the dual canonical basis and the dual semicanonical basis coincide.

\section{Case $\mathbb{A}_{5}$ : the tubular algebra $\Delta$ and the weighted projective line $\mathbb{X}$}

For the rest of this article we set $\Lambda=\Lambda_{5}, \widetilde{\Lambda}=\widetilde{\Lambda}_{5}, Q=Q_{5}, \Delta^{[a, b]}=\Delta_{5}^{[a, b]}, \Delta=\Delta_{5}=\Delta_{5}^{[0,1]}$ and $\mathbf{M}=\mathbf{M}(5)$. For our convenience we define moreover $\Delta^{[a]}=\Delta^{[a, a]}, \Delta_{0}=\Delta^{[0]}, \Delta_{\infty}=\Delta^{[1]}$ and $\Delta^{*}=\Delta^{[-1,0]}$. Note that $\Delta^{*} \cong \Delta^{\mathrm{op}}$.

14.1. Almost all components of the Auslander-Reiten quiver of $\Delta$ are tubes. This plays a crucial rôle in our results, so we shall recall the definition of a tube (see [1, p. 287], [45, p. 113]).

Let $\mathbb{Z} \mathbb{A}_{\infty}$ be the quiver with vertices $\left\{i_{j} \mid i \in \mathbb{Z}, j \in \mathbb{Z}^{+}\right\}$and arrows

$$
\left\{i_{j} \rightarrow i_{j+1} \mid i \in \mathbb{Z}, j \geqslant 1\right\} \cup\left\{i_{j} \rightarrow(i+1)_{j-1} \mid i \in \mathbb{Z}, j \geqslant 2\right\} .
$$

Define a map $\tau$ on the set of vertices by $\tau\left((i+1)_{j}\right)=i_{j}$. For a vertex $x$ in $\mathbb{Z} \mathbb{A}_{\infty}$ and $r \geqslant 1$ let

$$
[x]_{r}=\left\{\tau^{r i}(x) \mid i \in \mathbb{Z}\right\} .
$$

Then $T_{r}=\mathbb{Z} \mathbb{A}_{\infty} /\left(\tau^{r}\right)$ is the quiver with vertices $[x]_{r}$, and having an arrow $[x]_{r} \rightarrow[y]_{r}$ if and only if there is an arrow $x^{\prime} \rightarrow y^{\prime}$ for some $x^{\prime} \in[x]_{r}$ and some $y^{\prime} \in[y]_{r}$. The vertex $\left[i_{j}\right]_{r}$ in $T_{r}$ is said to have quasi-length $j$. The map $\tau$ induces a map on $T_{r}$ again denoted by $\tau$, given by

$$
\tau\left([x]_{r}\right)=[\tau(x)]_{r}
$$

and called the translation. In this way, $T_{r}$ becomes a translation quiver in the sense of [45, p. 47]. One calls $T_{r}$ a tube of rank $r$. A tube of rank 1 is called a homogeneous tube. The mouth of a tube is the subset of vertices of quasi-length 1 . Sometimes we also consider as a tube a translation quiver $\Gamma$ whose stable part $\underline{\Gamma}$, obtained by deleting the translates of the projective and injective vertices, is a tube.

14.2. For an algebra $A$, we call a class of indecomposable $A$-modules a tube if the vertices of $\Gamma_{A}$ that belong to that class form a tube [43, §3.1]. A family of tubes $\mathcal{T}=\left(\mathcal{T}_{x}\right)_{x \in X}$ is called a tubular $X$-family.

In our situation the index set $X$ will always be the projective line $\mathbb{P}_{1}(\mathbb{C})$. Such a tubular family is said to be of type $\left(m_{1}, \ldots, m_{n}\right)$ if for certain points $x_{1}, \ldots, x_{n}$ the corresponding tubes have rank $m_{1}, \ldots, m_{n}$ respectively, and for all remaining points the tubes are homogeneous. Because of these exceptional points, a better index set is provided by the weighted projective line $\mathbb{X}$ in the sense of Geigle-Lenzing [36], with exceptional points $x_{1}, \ldots, x_{n}$ having respective weights $m_{1}, \ldots, m_{n}$. Below we will point out some strong relations between the representation theory of $\Lambda$ and the weighted projective line $\mathbb{X}$ of weight type $\left(m_{1}, m_{2}, m_{3}\right)=(6,3,2)$ (see 14.6, 15.6).

$4^{\mathrm{e}}$ SÉRIE - TOME $38-2005-\mathrm{N}^{\circ} 2$ 
14.3. The algebra $\Delta_{\infty}$ is a tame concealed algebra of type $\widetilde{\mathbb{D}}_{6}$. This means that $\Delta_{\infty}$ is obtained from a hereditary algebra of type $\widetilde{\mathbb{D}}_{6}$ by tilting with respect to a preprojective tilting module [45, §4.3]. The tame concealed algebras have been classified by Happel and Vossieck [28], and one can check that $\Delta_{\infty}$ belongs to one of the frames in this list.

Similarly $\Delta_{0}$ is a tame concealed algebra of type $\widetilde{\mathbb{E}}_{7}$. Its tubular type is $(4,3,2)$ (see [45, p. 158]). There are two indecomposable $\Delta_{0}$-modules $M$ and $M^{\prime}$ completely determined (up to isomorphism) by their respective dimension vectors

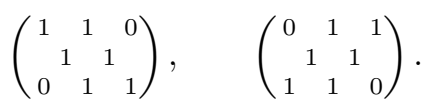

It can be shown that $M$ and $M^{\prime}$ lie at the mouth of a tube of the Auslander-Reiten quiver of $\Delta_{0}$, and that this tube has rank 4. Moreover, the tubular extension $\Delta_{0}[M]\left[M^{\prime}\right]$ (see $[45, \S 4.7]$ ) is isomorphic to $\Delta$. It follows that $\Delta$ is a tubular algebra of tubular type $(6,3,2)$ (see $[45, \S 5]$ ).

Similarly, $\Delta$ can be regarded as a tubular coextension of $\Delta_{\infty}$.

Note that the algebras $\Delta^{[2 i]}$ and $\Delta^{[2 i+1]}$ are isomorphic to $\Delta_{0}$ and $\Delta_{\infty}$, respectively, so they are tame concealed. Similarly the algebras $\Delta^{[2 i, 2 i+1]}$ and $\Delta^{[2 i-1,2 i]}$ are isomorphic to $\Delta$ and $\Delta^{*}$, respectively, hence they are tubular algebras. Thus we may speak of preprojective, regular and preinjective $\Delta^{[i]}$-modules or $\Delta^{[i, i+1]}$-modules.

14.4. We are going to define some tubular families of $\widetilde{\Lambda}$-modules. Following [27, §2] we introduce the following classes of modules. Let $\mathcal{T}^{[i]}$ be the class of indecomposable $\Delta^{[i-1, i+1]}$ modules $M$ such that the restriction of $M$ to $\Delta^{[i]}$ is regular and nonzero. Similarly, let $\mathcal{M}^{[i, i+1]}$ be the class of indecomposable $\Delta^{[i, i+1]}$-modules $M$ such that the restriction of $M$ to $\Delta^{[i]}$ is preinjective and the restriction to $\Delta^{[i+1]}$ is preprojective.

Clearly, we may also interpret the classes $\mathcal{T}^{[i]}$ and $\mathcal{M}^{[i, i+1]}$ as classes of $\widetilde{\Lambda}$-modules, on which the Galois group $\mathbb{Z}$ acts by

$$
{ }^{(j)} \mathcal{T}^{[i]}=\mathcal{T}^{[i+2 j]}, \quad{ }^{(j)} \mathcal{M}^{[i, i+1]}=\mathcal{M}^{[i+2 j, i+2 j+1]} \quad(j \in \mathbb{Z}) .
$$

The classes $\mathcal{T}_{0}=\mathcal{T}^{[0]}$ and $\mathcal{T}_{\infty}=\mathcal{T}^{[1]}$ are tubular families of type $(6,3,2)$. In Sections 20.6 and 20.7 we display the dimension vectors of the non-homogeneous tubes in these families. The remaining tubes consist only of the homogeneous $\Delta^{[i]}$-modules.

The classes $\mathcal{M}^{[i, i+1]}$ are the regular modules of the tubular algebra $\Delta^{[i, i+1]}$ which do not belong to the first or last tubular family. We may decompose $\mathcal{M}^{[i, i+1]}$ into a disjoint collection of tubular families $\left(\mathcal{T}_{\lambda}^{[i, i+1]}\right)_{\lambda \in \mathbb{Q}^{+}}$of type $(6,3,2)$ (see $[45, \S 5.2]$ and also 15.8 for more details).

14.5. Define

$$
\mathcal{H}=\mathcal{M}^{[-1,0]} \cup \mathcal{T}^{[0]} \cup \mathcal{M}^{[0,1]} \cup \mathcal{T}^{[1]}
$$

and

$$
H=\left\{\operatorname{dim}(M) \in K_{0}(\bmod (\widetilde{\Lambda})) \mid M \in \mathcal{H}\right\} \subset I(\widetilde{\Lambda}) .
$$

The next proposition shows that $\mathcal{H}$ is a transversal of the action of the Galois group on the set of indecomposable $\widetilde{\Lambda}$-modules.

PRoposition 14.1. - For each indecomposable $\widetilde{\Lambda}$-module $M$ there is a unique $j \in \mathbb{Z}$ such that ${ }^{(j)} M \in \mathcal{H}$. Moreover for each $\mathbf{d} \in I(\widetilde{\Lambda})$ there is a unique $i \in \mathbb{Z}$ with ${ }^{(i)} \mathbf{d} \in H$.

Proof. - By repeating the argument in $[27, \S 1]$ we find that each indecomposable module is contained in $\bigcup_{i \in \mathbb{Z}}\left(\mathcal{M}^{[i-1, i]} \cup \mathcal{T}^{[i]}\right)$. By (12) it only remains to show the unicity of $j$. This follows 
from the fact that the classes $\mathcal{M}^{[-1,0]}, \mathcal{T}^{[0]}, \mathcal{M}^{[0,1]}, \mathcal{T}^{[1]}$ are pairwise disjoint. Indeed $\mathcal{T}^{[0]}$ and $\mathcal{T}^{[1]}$ are disjoint as we can see from the dimension vectors of their objects, see 20.6 and 20.7. On the other hand $\mathcal{M}^{[-1,0]}$ and $\mathcal{M}^{[0,1]}$ are disjoint because a $\Delta^{[i]}$-module can not be preprojective and preinjective at the same time.

The second claim follows from the first. Indeed, we can determine the class $\mathcal{T}^{[i]}$ or $\mathcal{M}^{[i, i+1]}$ which an indecomposable module belongs to by means of $\operatorname{dim}(M)$ only (see [45, 5.2] and 15.8 below).

14.6. Proposition 14.1 shows in particular the (known) fact that $\widetilde{\Lambda}$ is locally support finite. Moreover the additive closure $\operatorname{add}(\mathcal{H})$ (i.e. the full subcategory of $\bmod (\widetilde{\Lambda})$ consisting of modules which are direct sums of modules of $\mathcal{H})$ is closed under Auslander-Reiten sequences. Thus by [17] and $[22, \S 3.6]$ we may identify the Auslander-Reiten quivers of $\operatorname{add}(\mathcal{H})$ and of $\bmod (\Lambda)$.

These considerations together with (11) imply that the image $\underline{\mathcal{H}}$ of $\mathcal{H}$ in $\underline{\bmod }(\widetilde{\Lambda})$ is a transversal for the indecomposable objects in $\underline{\bmod }(\widetilde{\Lambda})$ under the action of $\mathbb{Z}$ by powers of the translation functor $\Omega^{-1}$.

On the other hand, since $\Delta$ is a tubular algebra of tubular type $(6,3,2)$, we know from [23] that we have equivalences of triangulated categories

$$
\underline{\bmod }(\widetilde{\Lambda}) \cong D^{b}(\bmod (\Delta)) \cong D^{b}(\operatorname{coh}(\mathbb{X}))
$$

where $\mathbb{X}$ is a weighted projective line of weight type $(6,3,2)$. Since $\operatorname{coh}(\mathbb{X})$ is a hereditary category we have the useful decomposition

$$
D^{b}(\operatorname{coh}(\mathbb{X}))=\bigvee_{i \in \mathbb{Z}} \operatorname{coh}(\mathbb{X})[i]
$$

This is similar to the decomposition

$$
\underline{\bmod }(\widetilde{\Lambda})=\bigvee_{i \in \mathbb{Z}}(\operatorname{add}(\underline{\mathcal{H}}))[i]
$$

that we have just explained. Although a lot of our intuition on $\underline{\bmod }(\widetilde{\Lambda})$ comes from the comparison with $\operatorname{coh}(\mathbb{X})$, we prefer to avoid using this machinery.

\section{Case $\mathbb{A}_{5}$ : the root system}

15.1. Write $K_{0}(\Delta)$ for the Grothendieck group of $\bmod (\Delta)$. We have $K_{0}(\Delta)=\mathbb{Z}^{10}$ by identifying the class of a $\Delta$-module $M$ with its dimension vector $\operatorname{dim}(M) \in \mathbb{N}^{10}$.

Let $\langle-,-\rangle: \mathbb{Z}^{10} \times \mathbb{Z}^{10} \longrightarrow \mathbb{Z}$ be the Ringel bilinear form of $\Delta$, given by

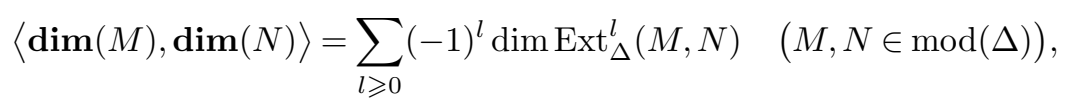

$4^{\mathrm{e}}$ SÉRIE - TOME $38-2005-\mathrm{N}^{\circ} 2$ 


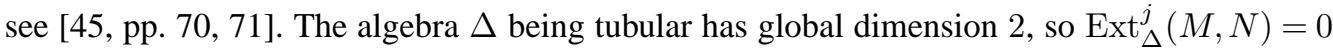
for $j>2$, and this infinite sum is in fact finite. Explicitly for

$$
\mathbf{d}=\left(\begin{array}{ccccc} 
& d_{2_{2}} & & d_{4_{1}} & \\
d_{1_{2}} & & d_{3_{1}} & & d_{5_{0}} \\
& d_{2_{1}} & & d_{4_{0}} & \\
d_{1_{1}} & & d_{3_{0}} & & d_{5_{-1}}
\end{array}\right), \quad \mathbf{e}=\left(\begin{array}{ccccc} 
& e_{2_{2}} & & e_{4_{1}} & \\
e_{1_{2}} & & e_{3_{1}} & & e_{5_{0}} \\
& e_{2_{1}} & & e_{4_{0}} & \\
e_{1_{1}} & & e_{3_{0}} & & e_{5_{-1}}
\end{array}\right)
$$

we have

$$
\langle\mathbf{d}, \mathbf{e}\rangle=\sum d_{i_{j}} e_{i_{j}}-\sum_{i_{j} \rightarrow k_{l}} d_{i_{j}} e_{k_{l}}+\sum_{i_{j}, k_{l}} r_{i_{j}, k_{l}} d_{i_{j}} e_{k_{l}}
$$

where the first sum runs over all indices $i_{j}$, the second one over all pairs $\left(i_{j}, k_{l}\right)$ such that there is an arrow from $i_{j}$ to $k_{l}$, and in the third sum $r_{i_{j}, k_{l}}$ is the number of relations from $i_{j}$ to $k_{l}$, i.e.

$$
r_{i_{j}, k_{l}}= \begin{cases}1 & \text { if }\left(i_{j}, k_{l}\right) \in\left\{\left(2_{2}, 2_{1}\right),\left(4_{1}, 4_{0}\right),\left(1_{2}, 1_{1}\right),\left(3_{1}, 3_{0}\right),\left(5_{0}, 5_{-1}\right)\right\} \\ 0 & \text { otherwise }\end{cases}
$$

15.2. Let $E$ be the matrix giving the Ringel form:

$$
\langle\mathbf{d}, \mathbf{e}\rangle=\mathbf{d}^{\mathrm{t}} E \mathbf{e},
$$

where $\mathbf{d}$ and $\mathbf{e}$ are interpreted as column vectors in $\mathbb{Z}^{10}$ and $\mathbf{d}^{\mathrm{t}}$ means transposition. Define the Coxeter matrix of $\Delta$ by $\Phi=-E^{-1} E^{\mathrm{t}}$ (see [45, p. 71]). It is easy to check that

$$
\langle\mathbf{d}, \mathbf{e}\rangle=-\langle\mathbf{e}, \Phi(\mathbf{d})\rangle=\langle\Phi(\mathbf{d}), \Phi(\mathbf{e})\rangle \quad\left(\mathbf{d}, \mathbf{e} \in \mathbb{Z}^{10}\right) .
$$

The data $\mathcal{L}=\left(\mathbb{Z}^{10},\langle-,-\rangle, \Phi\right)$ is called a bilinear lattice [34].

15.3. Let $\mathbf{d}=\left(d_{i_{j}}\right)_{1 \leqslant i \leqslant 5, j \in \mathbb{Z}}$ be an element of $K_{0}(\widetilde{\Lambda})=K_{0}(\bmod (\widetilde{\Lambda}))$. The support $\operatorname{supp}(M)$ of $M$ is defined as the set of vertices $i_{j}$ of $\widetilde{Q}$ such that the $i_{j}$ th component of $\operatorname{dim}(M)$ is nonzero. For $i \leqslant j$ it will be convenient to identify the Grothendieck group $K_{0}\left(\Delta^{[i, j]}\right)$ with the subgroup of $K_{0}(\widetilde{\Lambda})$ of elements with support in $\widetilde{Q}^{[i, j]}$, see 9.2 . We shall denote by $\langle-,-\rangle^{[i, j]}$ the Ringel form on $K_{0}\left(\Delta^{[i, j]}\right)$, so that $\langle-,-\rangle=\langle-,-\rangle^{[0,1]}$.

Let $\mathbf{p}_{1}, \mathbf{p}_{3}, \mathbf{p}_{5}$ (resp. $\mathbf{p}_{2}, \mathbf{p}_{4}$ ) be the dimension vectors of the indecomposable projective $\widetilde{\Lambda}$-modules with support in $\widetilde{Q}^{[0,2]}$ (resp. in $\left.\widetilde{Q}^{[-1,1]}\right)$, that is,

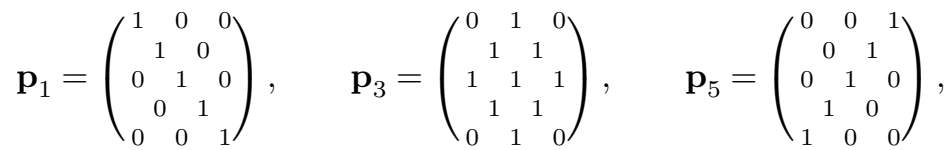

$$
\begin{aligned}
& \mathbf{p}_{2}=\left(\begin{array}{cccc}
1 & 1 & 0 \\
1 & 1 & 0 \\
& 1 & 1 & 1 \\
0 & 1 & 1 \\
0 & 1 & 1
\end{array}\right), \quad \mathbf{p}_{4}=\left(\begin{array}{cccc}
0 & 1 \\
0 & 1 & 1 \\
1 & 1 & 1 \\
1 & 1 & 0 \\
1 & 0
\end{array}\right) .
\end{aligned}
$$

Up to shift these are the dimension vectors of all indecomposable projective $\widetilde{\Lambda}$-modules. Note that these are precisely the dimension vectors of the projective $\widetilde{\Lambda}$-modules that belong to $\mathcal{H}$. The following result is verified by a direct calculation. 
LEMMA 15.1. - The map $K_{0}(\widetilde{\Lambda}) \longrightarrow K_{0}(\underline{\bmod }(\widetilde{\Lambda}))=K_{0}(\Delta), \operatorname{dim}(M) \mapsto[M]$ (see 9.9) induces isometries

$$
\begin{aligned}
& \iota_{+}: K_{0}\left(\Delta^{[-1,0]}\right) \longrightarrow K_{0}(\Delta), \mathbf{x} \mapsto \mathbf{x}-x_{4_{-1}} \mathbf{p}_{2}-x_{2_{0}} \mathbf{p}_{4}, \\
& \iota_{-}: K_{0}\left(\Delta^{[1,2]}\right) \longrightarrow K_{0}(\Delta), \quad \mathbf{x} \mapsto \mathbf{x}-x_{1_{3}} \mathbf{p}_{1}-x_{3_{2}} \mathbf{p}_{3}-x_{5_{1}} \mathbf{p}_{5},
\end{aligned}
$$

of bilinear lattices, that is,

$$
\langle\mathbf{x}, \mathbf{y}\rangle^{[-1,0]}=\left\langle\iota_{+}(\mathbf{x}), \iota_{+}(\mathbf{y})\right\rangle, \quad\langle\mathbf{x}, \mathbf{y}\rangle^{[1,2]}=\left\langle\iota_{-}(\mathbf{x}), \iota_{-}(\mathbf{y})\right\rangle .
$$

15.4. Let $q^{[i, j]}: K_{0}\left(\Delta^{[i, j]}\right) \longrightarrow \mathbb{Z}$ be the quadratic form $q^{[i, j]}(\mathbf{d})=\langle\mathbf{d}, \mathbf{d}\rangle^{[i, j]}$. For simplicity we write $q^{[i]}=q^{[i, i]}$ and $q=q^{[0,1]}$. Define $\mathbf{h}^{[i]}$ to be the positive generator of the one-dimensional radical of $q^{[i]}$ (recall that $\Delta^{[i]}$ is tame concealed). This means for example that the support of $\mathbf{h}^{[0]}$ and of $\mathbf{h}^{[1]}$ is contained in $\widetilde{Q}^{[0,1]}$. Explicitly

$$
\mathbf{h}^{[0]}=\left(\begin{array}{cccc}
0 & 0 & 0 \\
1 & 2 & 1 \\
3 & 3 & 3 \\
1 & 2 & 1
\end{array}\right), \quad \mathbf{h}^{[1]}=\left(\begin{array}{ccc}
1 & 1 & 1 \\
1 & 2 & 1 \\
1 & 1 & 1 \\
0 & 0 & 0
\end{array}\right) .
$$

Moreover $\mathbf{h}^{[2 i]}={ }^{(i)} \mathbf{h}^{[0]}$ and $\mathbf{h}^{[2 i+1]}={ }^{(i)} \mathbf{h}^{[1]}$. Since $q^{[i, i+1]}$ is positive semidefinite of corank 2 it is easy to see that $\operatorname{rad}\left(q^{[i, i+1]}\right)=\mathbb{Z} \mathbf{h}^{[i]} \oplus \mathbb{Z} \mathbf{h}^{[i+1]}$. Notice that (Lemma 15.1)

$$
\iota_{+}\left(\mathbf{h}^{[-1]}\right)=-\mathbf{h}^{[1]}, \quad \iota_{+}\left(\mathbf{h}^{[0]}\right)=\mathbf{h}^{[0]} .
$$

It will be convenient to set $\mathbf{h}_{0}=\mathbf{h}^{[0]}$ and $\mathbf{h}_{\infty}=\mathbf{h}^{[1]}$. These are the two generators of $\operatorname{rad}(q)$.

15.5. Let

$$
R=\left\{\mathbf{d} \in \mathbb{Z}^{10} \mid q(\mathbf{d})=0,1, \mathbf{d} \neq 0\right\}
$$

be the set of roots of $q$. A root $\mathbf{d} \in R$ is called imaginary if $q(\mathbf{d})=0$, and real otherwise. Let $R^{\mathrm{im}}$ (resp. $R^{\mathrm{re}}$ ) be the set of imaginary (resp. real) roots of $q$.

The form $q$ being positive semidefinite, $R^{\mathrm{im}}$ consists of the nonzero elements of $\operatorname{rad}(q)$. Note that, by (13), $\mathbf{d}$ is a radical vector if and only if $\Phi(\mathbf{d})=\mathbf{d}$. Indeed,

$$
\langle\mathbf{d}, \mathbf{e}\rangle+\langle\mathbf{e}, \mathbf{d}\rangle=\langle\mathbf{d}-\Phi(\mathbf{d}), \mathbf{e}\rangle
$$

and the Ringel form $\langle-,-\rangle$ is nondegenerate.

Since $\Delta$ has tubular type $(6,3,2)$, the form $q$ induces a positive definite form of type $\mathbb{E}_{8}$ on $\mathbb{Z}^{10} / \operatorname{rad}(q)$. (Note that the Dynkin diagram of type $\widetilde{\mathbb{E}}_{8}$ is a star with three branches of lengths $6,3,2$.) Thus, $R$ is an elliptic root system of type $\mathbb{E}_{8}^{(1,1)}$ in the sense of Saito [50].

15.6. By [45, p. 278], the elements of $R \cap \mathbb{N}^{10}$ are precisely the dimension vectors of the indecomposable $\Delta$-modules. Note however that there exist elements in $R$ whose coordinates are not all nonnegative or all nonpositive. For example

$$
\left(\begin{array}{cccc}
-1 & 0 & \\
0 & & 0 & 0 \\
& 1 & 0 & \\
0 & & 0 & 0
\end{array}\right) \in R
$$

$4^{e}$ SÉRIE - TOME $38-2005-\mathrm{N}^{\circ} 2$ 
Following [36] and [24] we will introduce another notion of positive root in $R$. Define the subset of positive roots of $q$ as

$$
R^{+}=\left\{\mathbf{r} \in R \mid\left\langle\mathbf{r}, \mathbf{h}_{\infty}\right\rangle>0 \text { or }\left(\left\langle\mathbf{r}, \mathbf{h}_{\infty}\right\rangle=0 \text { and }\left\langle\mathbf{h}_{0}, \mathbf{r}\right\rangle>0\right)\right\},
$$

and set $R^{-}=\left\{-\mathbf{d} \mid \mathbf{d} \in R^{+}\right\}$.

In our setting $R^{+}$is the set of classes in $K_{0}(\Delta)=K_{0}(\bmod (\widetilde{\Lambda}))$ of the non-projective (indecomposable) $\widetilde{\Lambda}$-modules which belong to $\mathcal{H}$ as we will see in Corollary 15.3. Then it follows from 14.6 that $R=R^{+} \cup R^{-}$, see also [36]. One could also derive this fact from a careful analysis of $R$.

Our definition of $R^{+}$is partially inspired by the interpretation of the bilinear lattice $\mathcal{L}$ as the Grothendieck group of the category $\operatorname{coh}(\mathbb{X})$, see 14.6. Indeed, since $\operatorname{coh}(\mathbb{X})$ and $\bmod (\Delta)$ are derived equivalent [36], they have isomorphic Grothendieck groups [26, p. 101]. The linear forms $\left\langle-, \mathbf{h}_{\infty}\right\rangle$ and $\left\langle\mathbf{h}_{0},-\right\rangle$ correspond to the functions "rank" and "degree" on $K_{0}(\operatorname{coh}(\mathbb{X}))$, and $R^{+}$corresponds to the set of classes of indecomposable sheaves.

15.7. Since for $\mathbf{d} \in R^{+}$we have $\left\langle\mathbf{d}, \mathbf{h}_{\infty}\right\rangle \neq 0$ or $\left\langle\mathbf{h}_{0}, \mathbf{d}\right\rangle \neq 0$, the quotient $\left\langle\mathbf{h}_{0}, \mathbf{d}\right\rangle /\left\langle\mathbf{d}, \mathbf{h}_{\infty}\right\rangle$ is a well-defined element of $\mathbb{Q}_{\infty}$. For $\lambda \in \mathbb{Q}_{\infty}$ let $R^{\lambda}=\left\{\mathbf{d} \in R^{+} \mid\left\langle\mathbf{h}_{0}, \mathbf{d}\right\rangle /\left\langle\mathbf{d}, \mathbf{h}_{\infty}\right\rangle=\lambda\right\}$ be the set of positive roots of slope $\lambda$. Thus

$$
R^{+}=\bigcup_{\lambda \in \mathbb{Q}_{\infty}} R^{\lambda}
$$

For $a \in \mathbb{N}$ and $b \in \mathbb{Z}$ such that $a>0$ or $(a=0$ and $b>0)$ set

$$
\mathbf{h}_{a, b}=a \mathbf{h}_{0}+b \mathbf{h}_{\infty} .
$$

We have $R^{\lambda} \cap R^{\mathrm{im}}=\left\{\mathbf{h}_{a, b} \mid b / a=\lambda\right\}$. This follows immediately from the equalities

$$
\left\langle\mathbf{h}_{0}, \mathbf{h}_{\infty}\right\rangle=-\left\langle\mathbf{h}_{\infty}, \mathbf{h}_{0}\right\rangle=6, \quad\left\langle\mathbf{h}_{0}, \mathbf{h}_{0}\right\rangle=\left\langle\mathbf{h}_{\infty}, \mathbf{h}_{\infty}\right\rangle=0 .
$$

Set

$$
R^{++}=\bigcup_{\lambda \in \mathbb{Q}^{+}} R^{\lambda}, \quad R^{+-}=\bigcup_{\lambda \in \mathbb{Q}^{-}} R^{\lambda} .
$$

Thus $R^{+}=R^{+-} \cup R^{0} \cup R^{++} \cup R^{\infty}$.

15.8. The above decomposition of $R^{+}$is motivated by the following fact. For $\lambda \in \mathbb{Q}^{+}$let $\mathcal{T}_{\lambda}^{[i, i+1]}$ be the class of indecomposable $\Delta^{[i, i+1]}$-modules $M$ with

$$
\frac{\left\langle\mathbf{h}^{[i]}, \operatorname{dim}(M)\right\rangle^{[i, i+1]}}{\left\langle\operatorname{dim}(M), \mathbf{h}^{[i+1]}\right\rangle^{[i, i+1]}}=\lambda .
$$

Then $\mathcal{T}_{\lambda}^{[i, i+1]}$ is a tubular family of type $(6,3,2)$ and $\bigcup_{\lambda \in \mathbb{Q}^{+}} \mathcal{T}^{[i, i+1]}=\mathcal{M}^{[i, i+1]}$. Moreover the set of dimension vectors $\left\{\operatorname{dim}(M) \mid M \in \mathcal{T}_{\lambda}^{[i, i+1]}\right\}$ coincides with the set of roots $\mathbf{v}$ of $K_{0}\left(\Delta^{[i, i+1]}\right)$ such that

$$
\frac{\left\langle\mathbf{h}^{[i]}, \mathbf{v}\right\rangle^{[i, i+1]}}{\left\langle\mathbf{v}, \mathbf{h}^{[i+1]}\right\rangle^{[i, i+1]}}=\lambda,
$$

ANNALES SCIENTIFIQUES DE L’ÉCOLE NORMALE SUPÉRIEURE 
see $[45,5.2]$. For $\lambda \in \mathbb{Q}_{\infty}$ we may now define the tubular family

$$
\mathcal{T}_{\lambda}= \begin{cases}\mathcal{T}^{[1]} & \text { for } \lambda=\infty, \\ \mathcal{T}_{\lambda}^{[0,1]} & \text { for } 0<\lambda<\infty, \\ \mathcal{T}^{[0]} & \text { for } \lambda=0, \\ \mathcal{T}_{-1 / \lambda}^{[-1,0]} & \text { for } \lambda<0 .\end{cases}
$$

Clearly we have $\bigcup_{\lambda \in \mathbb{Q}_{\infty}} \mathcal{T}_{\lambda}=\mathcal{H}$. We shall denote by $T_{\lambda, x}$ the tube of the family $\mathcal{T}_{\lambda}$ indexed by $x \in \mathbb{X}$. Thus, for all points $x$ except the three exceptional points $x_{1}, x_{2}, x_{3}, T_{\lambda, x}$ is a homogeneous tube, and $T_{\lambda, x_{1}}, T_{\lambda, x_{2}}, T_{\lambda, x_{3}}$ have respective ranks 6,3 and 2 .

Lemma 15.2. - For $\lambda \in \mathbb{Q}_{\infty}$ we have $R^{\lambda}=\left\{[M] \mid M \in \mathcal{T}_{\lambda}\right.$ and $M$ is non-projective $\}$.

Proof. - For $\lambda \in\{0, \infty\}$ this may be verified directly by comparing the dimension vectors of the objects in $\mathcal{T}_{0}$ (resp. $\mathcal{T}_{\infty}$ ) which we describe in 20.6 (resp. 20.7) with the classes in $R^{0}$ (resp. $R^{\infty}$ ) which we can construct explicitly, see Theorem 15.5. For $0<\lambda<\infty$ the result is clear from the case $i=0$ of Ringel's result which we explained above. For $\lambda<0$ it follows from the same result for $i=-1$ after applying the isometry $\iota_{+}$from Lemma 15.1 and (14).

COROLlaRY 15.3. - The restriction $\xi: H \longrightarrow R^{+} \cup\{0\}$ of the map $K_{0}(\widetilde{\Lambda}) \longrightarrow K_{0}(\Delta)$, $\operatorname{dim}(M) \mapsto[M]$, is well-defined and surjective. Moreover, we have

$$
\begin{aligned}
& \xi^{-1}(0)=\left\{\mathbf{p}_{1}, \mathbf{p}_{2}, \mathbf{p}_{3}, \mathbf{p}_{4}, \mathbf{p}_{5}\right\}, \\
& \xi^{-1}\left(m \mathbf{h}_{0}\right)=\left\{m \mathbf{h}_{0}, m \mathbf{h}_{0}+\mathbf{p}_{2}, m \mathbf{h}_{0}+\mathbf{p}_{4}\right\} \quad\left(m \in \mathbb{Z}^{+}\right), \\
& \xi^{-1}\left(m \mathbf{h}_{\infty}\right)=\left\{m \mathbf{h}_{\infty}, m \mathbf{h}_{\infty}+\mathbf{p}_{1}, m \mathbf{h}_{\infty}+\mathbf{p}_{3}, m \mathbf{h}_{\infty}+\mathbf{p}_{5}\right\} \quad\left(m \in \mathbb{Z}^{+}\right) . \\
& \text {If } \mathbf{r} \in R^{+} \backslash\left\{\mathbb{Z}^{+} \mathbf{h}_{0} \cup \mathbb{Z}^{+} \mathbf{h}_{\infty}\right\} \text { the set } \xi^{-1}(\mathbf{r}) \text { contains precisely one element. }
\end{aligned}
$$

We leave the proof to the reader.

15.9. Since $\Delta$ is tubular of tubular type $(6,3,2)$, we have that $\Phi^{6}=1$ (see $[34,35]$ ). Of course this can also be verified directly by a simple calculation.

Define the rank $\operatorname{rk}(\mathbf{d})$ of a vector $\mathbf{d} \in \mathbb{Z}^{10}$ as the minimal $n \geqslant 1$ such that $\Phi^{n}(\mathbf{d})=\mathbf{d}$. Hence, if $\mathbf{d}$ is an imaginary $\operatorname{root} \operatorname{rk}(\mathbf{d})=1$, and if $\mathbf{d}$ is a real $\operatorname{root} \operatorname{rk}(\mathbf{d})=2,3$ or 6 .

The quasi-length $\mathrm{ql}(\mathbf{d})$ of $\mathbf{d}$ is defined as the greatest common divisor of the entries in the imaginary root

$$
h(\mathbf{d})=\sum_{i=1}^{\mathrm{rk}(\mathbf{d})} \Phi^{i}(\mathbf{d}) .
$$

In other words, writing $h(\mathbf{d})=\mathbf{h}_{a, b}$ we have $\mathrm{ql}(\mathbf{d})=\operatorname{gcd}(a, b)$. Set also

$$
\operatorname{iso}(\mathbf{d})=\frac{1}{\mathrm{ql}(\mathbf{d})} h(\mathbf{d}) \text {. }
$$

Then $\mathbf{d} \in R^{\lambda}$ if and only if iso( $\left.\mathbf{d}\right)=\mathbf{h}_{a, b}$ where $\lambda=b / a$ and $\operatorname{gcd}(a, b)=1$.

These definitions are motivated by the fact that for a non-projective (indecomposable) $\widetilde{\Lambda}$-module $M$ which has quasi-length $\ell$ in a tube of rank $r$ we have

$$
\mathrm{ql}([M])=\left\{\begin{array}{ll}
\ell & \text { if } r \nmid \ell, \\
\ell / r & \text { if } r \mid \ell,
\end{array} \quad \operatorname{rk}([M])= \begin{cases}r & \text { if } r \nmid \ell, \\
1 & \text { if } r \mid \ell .\end{cases}\right.
$$

$4^{e}$ SÉRIE - TOME $38-2005-\mathrm{N}^{\circ} 2$ 
(Of course our rk should not be confused with the rank function on $K_{0}(\operatorname{coh}(\mathbb{X}))$.)

15.10. For $\lambda \in \mathbb{Q}_{\infty}, i \in\{1,2,3,6\}$ and $\ell \in \mathbb{Z}^{+}$let

$$
R_{\ell}^{\lambda}(i)=\left\{\mathbf{d} \in R^{\lambda} \mid \operatorname{rk}(\mathbf{d})=i, \operatorname{ql}(\mathbf{d})=\ell\right\}
$$

so that

$$
R^{\lambda}=\bigcup_{\ell \in \mathbb{Z}^{+}, i \in\{1,2,3,6\}} R_{\ell}^{\lambda}(i) .
$$

LEMMA 15.4. - (a) If $\mathbf{d} \in R_{\ell}^{\lambda}(i)$ then $R_{\ell}^{\lambda}(i)=\left\{\Phi^{j}(\mathbf{d}) \mid j=1, \ldots, i\right\}$;

(b) If $i>1$ and $i$ divides $\ell$, then $R_{\ell}^{\lambda}(i)=\emptyset$;

(c) If $i>1$ and $i$ does not divide $\ell$, then $R_{\ell}^{\lambda}(i)$ consists of $i$ real roots;

(d) If $i=1$, then $R_{\ell}^{\lambda}(1)=\left\{h_{a, b}\right\}$ where $b / a=\lambda$ and $\operatorname{gcd}(a, b)=\ell$.

Proof. - Let $\mathbf{d} \in R_{\ell}^{\lambda}(i)$. Clearly, $\operatorname{rk}(\Phi(\mathbf{d}))=\operatorname{rk}(\mathbf{d}), h(\Phi(\mathbf{d}))=h(\mathbf{d})$, and $\mathrm{ql}(\Phi(\mathbf{d}))=\mathrm{ql}(\mathbf{d})$. Moreover, by Eq. (13) we have $\Phi(\mathbf{d}) \in R^{\lambda}$. Hence the $\Phi$-orbit of $\mathbf{d}$ is contained in $R_{\ell}^{\lambda}(i)$.

Conversely, if $R_{\ell}^{\lambda}(i)$ is nonempty, then it consists of a single $\Phi$-orbit. This follows from (10) and Lemma 15.2 (remember that $\mathcal{T}_{\lambda}$ is a tubular family of type $(6,3,2)$ ). Note that all the indecomposable modules lying on some homogeneous tube of $\mathcal{T}_{\lambda}$ and having the same quasilength $\ell$ have the same class $\mathbf{h}_{a, b}$ in $K_{0}(\Delta)$, where $b / a=\lambda$ and $\operatorname{gcd}(a, b)=\ell$. Moreover for $i=2,3,6$ the element $\mathbf{h}_{a, b}$ is also the class of $i$ modules with quasi-length $\ell$ lying on the tube of rank $i$ of $\mathcal{T}_{\lambda}$. It follows that there is no positive root $\mathbf{d}$ with $\operatorname{rk}(\mathbf{d})=i>1$ and $\mathrm{ql}(\mathbf{d})$ divisible by $i$.

15.11. Let $i \in\{2,3,6\}$. For $0 \leqslant m, n \leqslant i-1$ and $(m, n) \neq(0,0)$ set

$$
R_{[m, n]}(i)=R_{\ell}^{\lambda}(i)
$$

where $\lambda=n / m$ and $\ell=\operatorname{gcd}(m, n)$. By Lemma 15.4, $R_{[m, n]}(i)$ has cardinality $i$. In Section 20.8 we list all elements of the sets $R_{[m, n]}(i)$, that is, a distinguished set of

$$
2 \cdot 3+3 \cdot 8+6 \cdot 35=240
$$

real elements, say $\mathbf{r}_{1}, \ldots, \mathbf{r}_{240}$, of $R^{+}$.

We constructed this set in the following way. Note first, that it coincides with the set

$$
\left\{\mathbf{r} \in R^{+} \mid 0 \leqslant\left\langle\mathbf{h}_{0}, \mathbf{r}\right\rangle,\left\langle\mathbf{r}, \mathbf{h}_{\infty}\right\rangle \leqslant 5\right\} .
$$

Now consider the algebra $\Delta^{\prime}$ which is obtained by restricting $\Delta$ to the full subquiver of $\widetilde{Q}^{[0,1]}$ which is obtained by removing the vertices $1_{1}$ and $4_{1}$. Notice that if $\mathbf{r}$ is a real root, then $\mathbf{r}-r_{1_{1}} \mathbf{h}_{0}-r_{4_{1}} \mathbf{h}_{\infty}$ is a real root with support in $\Delta^{\prime}$. This is tilted of type $\mathbb{E}_{8}$, thus the associated quadratic form $q^{\prime}$ has 240 roots $\mathbf{r}_{1}^{\prime}, \ldots, \mathbf{r}_{240}^{\prime}$. Find them for example as the orbits of the dimension vectors of the 8 indecomposable projective $\Delta^{\prime}$-modules under the corresponding Coxeter transformation $\Phi^{\prime}$. Since $\Delta^{\prime}$ is tilted of type $\mathbb{E}_{8}$, the transformation $\Phi^{\prime}$ has order 30 (the Coxeter number of $\mathbb{E}_{8}$ ). Next, define integers $a_{i}^{\prime}, a_{i}^{\prime \prime}, b_{i}^{\prime}, b_{i}^{\prime \prime}$ by

$$
\begin{aligned}
\left\langle\mathbf{r}_{i}^{\prime}, \mathbf{h}_{\infty}\right\rangle & =6 a_{i}^{\prime}+a_{i}^{\prime \prime} \quad \text { with } 0 \leqslant a_{i}^{\prime \prime} \leqslant 5 \\
\left\langle\mathbf{h}_{0}, \mathbf{r}_{i}^{\prime}\right\rangle & =6 b_{i}^{\prime}+b_{i}^{\prime \prime} \quad \text { with } 0 \leqslant b_{i}^{\prime \prime} \leqslant 5,
\end{aligned}
$$


for $1 \leqslant i \leqslant 240$. Finally set $\mathbf{r}_{i}=\mathbf{r}_{i}^{\prime}-a_{i}^{\prime} \mathbf{h}_{0}-b_{i}^{\prime} \mathbf{h}_{\infty}$.

We can now give the following explicit construction of all real roots in $R^{+}$.

THEOREM 15.5 (Construction of $R_{\ell}^{\lambda}(i)$ ). - Let $\lambda \in \mathbb{Q}_{\infty}, i \in\{2,3,6\}$ and $\ell \in \mathbb{Z}^{+}$not divisible by $i$. Write $\lambda=b / a$ with $a \in \mathbb{N}, b \in \mathbb{Z}$ and $\operatorname{gcd}(a, b)=\ell$ (if $\lambda=\infty$ set $a=0$ and $b=\ell$ ). Write

$$
a=i a^{\prime}+a^{\prime \prime}, \quad b=i b^{\prime}+b^{\prime \prime}
$$

with $a^{\prime}, a^{\prime \prime}, b^{\prime}, b^{\prime \prime} \in \mathbb{Z}$ such that $0 \leqslant a^{\prime \prime}, b^{\prime \prime} \leqslant i-1$. Then

$$
R_{\ell}^{\lambda}(i)=\left\{\mathbf{h}_{a^{\prime}, b^{\prime}}+\mathbf{r} \mid \mathbf{r} \in R_{\left[a^{\prime \prime}, b^{\prime \prime}\right]}(i)\right\}
$$

Proof. - Let $\mathbf{r}$ be a real root of rank $i, \mathbf{h}$ an imaginary root and set $\mathbf{d}=\mathbf{r}+\mathbf{h}$. Then $\mathbf{d}$ is a real root and $\Phi(\mathbf{d})=\Phi(\mathbf{r})+\mathbf{h}$, hence $\mathbf{d}$ has also rank $i$. Moreover, if $\mathbf{h}=\mathbf{h}_{a^{\prime}, b^{\prime}}$ and $\mathbf{r} \in R_{\left[a^{\prime \prime}, b^{\prime \prime}\right]}(i)$ then $h(\mathbf{d})=i \mathbf{h}_{a^{\prime}, b^{\prime}}+\mathbf{h}_{a^{\prime \prime}, b^{\prime \prime}}=\mathbf{h}_{a, b}$, therefore $\mathbf{d}$ belongs to $R_{\ell}^{\lambda}(i)$. Thus, since $R_{\ell}^{\lambda}(i)$ and $R_{\left[a^{\prime \prime}, b_{i}\right]}(i)$ both have cardinality $i$ we see that the first subset is the translate of the second one by $\mathbf{h}_{a^{\prime}, b^{\prime}}$.

Thus, the 240 positive real roots listed in Section 20.8 yield a complete description of the infinite set of all positive real roots of $R$. Note that the classes of these 240 roots in $\mathbb{Z}^{10} / \operatorname{rad}(q)$ form a finite root system of type $\mathbb{E}_{8}$, and we recover that $R$ is an elliptic root system of type $\mathbb{E}_{8}^{(1,1)}$.

15.12. The set $R_{S}^{+}$of Schur roots is defined as

$$
R_{S}^{+}=\left\{\mathbf{d} \in R^{+} \mid \operatorname{gcd}\left(\left\langle\mathbf{h}_{0}, \mathbf{d}\right\rangle,\left\langle\mathbf{d}, \mathbf{h}_{\infty}\right\rangle\right) \leqslant 6\right\} .
$$

It is easy to see that the set of imaginary Schur roots consists of the $h_{a, b}$ with $\operatorname{gcd}(a, b)=1$.

The Schur roots can also be characterized in terms of rank and quasi-length, namely

$$
R_{S}^{+}=\left\{\mathbf{d} \in R^{+} \mid \mathrm{ql}(\mathbf{d}) \leqslant \operatorname{rk}(\mathbf{d})\right\} .
$$

This comes from the identity

$$
\operatorname{gcd}\left(\left\langle\mathbf{h}_{0}, \mathbf{d}\right\rangle,\left\langle\mathbf{d}, \mathbf{h}_{\infty}\right\rangle\right)=6 \frac{\mathrm{ql}(\mathbf{d})}{\operatorname{rk}(\mathbf{d})} .
$$

Thus the set of real Schur roots is equal to the union of all subsets $R_{\ell}^{\lambda}(i)$ for $\lambda \in \mathbb{Q}_{\infty}, i=2,3,6$ and $1 \leqslant \ell<i$. Using 15.10, this implies that $R_{S}^{+} \cap R^{\lambda}$ contains exactly

$$
5 \cdot 6+2 \cdot 3+1 \cdot 2+1=39
$$

roots, one imaginary and the others real. Note that all the 240 roots listed in Section 20.8 are Schur roots.

The Schur roots of $R^{+}$are related to the Schur roots of $\widetilde{\Lambda}$ (see 10.1) in the following way. Suppose that $M \in \mathcal{H}$ is non-projective and has quasi-length $\ell$ in a tube of rank $r$. Since all tubes in $\Gamma_{\widetilde{\Lambda}}$ are standard, the endomorphism ring of $M$ is non-trivial if and only if either $\ell>r$, or $\ell=r$ and $M$ has a non-trivial endomorphism that factors over a projective module in the same tube.

$4^{\mathrm{e}}$ SÉRIE - TOME $38-2005-\mathrm{N}^{\circ} 2$ 
COROLlary 15.6. - A module $M \in \mathcal{H}$ has trivial endomorphism ring if and only if $[M]=0$, or $[M] \in R_{S}^{+}$and $\operatorname{dim}(M)$ does not belong to the following list:

$$
\mathbf{h}_{\infty}+\mathbf{p}_{i}, \quad \mathbf{h}_{0}+\mathbf{p}_{j}
$$

with $i=1,3,5$ and $j=2,4$.

Proof. - This follows from (15) together with our description of $\mathcal{T}_{0}$ and $\mathcal{T}_{\infty}$.

\section{Case $\mathbb{A}_{5}$ : parametrization of the indecomposable irreducible components}

We shall now explain how $R^{+}$parametrizes (i) the indecomposable $\Lambda$-modules and (ii) the set of dimension vectors of indecomposable $\widetilde{\Lambda}$-modules modulo the Galois group action. From (ii) we shall deduce the main result of this section, namely the parametrization of the indecomposable irreducible components of $\Lambda$-modules by $R_{S}^{+}$.

16.1. By 14.6, the indecomposable $\Lambda$-modules are in one-to-one correspondence with the $\widetilde{\Lambda}$-modules of the class $\mathcal{H}$. This class decomposes into tubular families $\mathcal{T}_{\lambda}$ as shown in 15.8. The projective modules $P_{1}$ and $P_{5}$ appear at the mouth of the non-homogeneous tube $T_{\infty, x_{1}}$, the module $P_{3}$ at the mouth of the non-homogeneous tube $T_{\infty, x_{2}}$, and the modules $P_{2}$ and $P_{4}$ at the mouth of the non-homogeneous tube $T_{0, x_{1}}$. As a result we obtain the following parametrization of the indecomposable $\Lambda$-modules by $R^{+}$.

Proposition 16.1. - Let $\lambda \in \mathbb{Q}_{\infty}, i \in\{1,2,3,6\}$ and $\ell \in \mathbb{Z}^{+}$. Then the following hold:

(a) If $i$ does not divide $\ell$ then there exists a one-to-one correspondence between $R_{\ell}^{\lambda}(i)$ and the set of indecomposable $\Lambda$-modules of quasi-length $\ell$ in the non-homogeneous tube $T_{\lambda, x_{j}}$ with $m_{j}=i$. This correspondence maps $\mathbf{d} \in R_{\ell}^{\lambda}(i)$ to $M=F(N)$ with $[N]=\mathbf{d}$.

(b) If $i=1$ then $R_{\ell}^{\lambda}(1)=\left\{\mathbf{h}_{a, b}\right\}$ where $b / a=\lambda$ and $\operatorname{gcd}(a, b)=\ell$. There is an infinite set of indecomposable $\Lambda$-modules $M=F(N)$ with $[N]=\mathbf{h}_{a, b}$, parametrized by the weighted projective line $\mathbb{X}$. More precisely, for each ordinary point $x \in \mathbb{X}$ there is an indecomposable module of quasi-length $\ell$ in the tube $T_{\lambda, x}$, and for each exceptional point $x_{j}$, there are $m_{j}$ indecomposable modules of quasi-length $\ell$ on $T_{\lambda, x_{j}}$.

(c) The only indecomposable $\Lambda$-modules not appearing in the above lists are the five indecomposable projective modules.

16.2. Recall from Corollary 15.3 that we have a canonical map $\xi: H \rightarrow R^{+} \cup\{0\}$. We are going to define a 'right inverse' $\delta$ of $\xi$. Define $\underline{\delta}: R^{+} \longrightarrow K_{0}(\widetilde{\Lambda})$ by

$$
\underline{\delta}(\mathbf{r})= \begin{cases}\mathbf{r}-\min \left\{0, r_{2_{2}}\right\} \mathbf{p}_{2}-\min \left\{0, r_{4_{1}}\right\} \mathbf{p}_{4} & \text { if } \mathbf{r} \in R^{\infty}, \\ \mathbf{r} & \text { if } \mathbf{r} \in R^{++}, \\ \mathbf{r}-\min \left\{0, r_{1_{1}}\right\} \mathbf{p}_{1}-\min \left\{0, r_{3_{0}}\right\} \mathbf{p}_{3}-\min \left\{0, r_{5_{-1}}\right\} \mathbf{p}_{5} & \text { if } \mathbf{r} \in R^{0}, \\ \mathbf{r}-r_{2_{2}} \mathbf{p}_{2}-r_{4_{1}} \mathbf{p}_{4} & \text { if } \mathbf{r} \in R^{+-} .\end{cases}
$$

PROPOSITION 16.2. - With the above definition of $\underline{\delta}$ we have:

(a) $\underline{\delta}$ induces a well-defined and injective map $\delta: R^{+} \longrightarrow H \cong I(\widetilde{\Lambda}) / \sim$;

(b) The only elements of $H$ not in the image of $\delta$ are

$$
\mathbb{N h}_{\infty}+\mathbf{p}_{i}, \quad \mathbb{N h}_{0}+\mathbf{p}_{j}
$$

with $i=1,3,5$ and $j=2,4$; 
(c) The map $\delta$ restricts to a map $\delta_{S}: R_{S}^{+} \longrightarrow H_{S}=H \cap R_{S}^{+}$. The only elements of $H_{S}$ not in the image of $\delta_{S}$ are $\mathbf{p}_{1}, \ldots, \mathbf{p}_{5}$.

Proof. - First we have to show that $\underline{\delta}(\mathbf{r})$ is the dimension vector of an indecomposable $\widetilde{\Lambda}$ module. This is clear for $\mathbf{r} \in R^{++}$. For $\mathbf{r} \in R^{+-}$we notice that $\mathbf{x} \mapsto \mathbf{x}-x_{2_{2}} \mathbf{p}_{2}-x_{4_{1}} \mathbf{p}_{4}$ gives an isometry $K_{0}(\Delta) \longrightarrow K_{0}\left(\Delta^{[-1,0]}\right)$, so that $\underline{\delta}\left(R^{+-}\right)$consists of dimension vectors of objects in $\mathcal{M}^{[-1,0]}$. The remaining two cases are treated directly. It is easy to calculate the map $\xi$ (Corollary 15.3) explicitly with (9). It follows that $\xi \underline{\delta}=\mathbf{1}_{R^{+}}$and the rest of (a) follows since $H$ is a transversal for the action of $\mathbb{Z}$ on $\operatorname{ind}(\widetilde{\Lambda})$, see Proposition 14.1. Now we obtain (b) from the description of the fibres of $\xi$ in Corollary 15.3, and (c) follows from Corollary 15.6.

16.3. Collecting the results of Theorem 10.1, Theorem 10.3 and Proposition 16.2 we can now state the following parametrization of the indecomposable irreducible components of varieties of $\Lambda$-modules and of the corresponding multisegments. Let $C_{j}(1 \leqslant j \leqslant 5)$ be the irreducible components containing the five indecomposable projective $\Lambda$-modules. Let $\mathfrak{p}_{j}$ denote the corresponding multisegments, namely

$$
\mathfrak{p}_{j}=\sum_{i=1}^{5-j+1}[i, i+j-1] \quad(j=1, \ldots, 5) .
$$

THEOREM 16.3. - (a) The map

$$
\mathbf{d} \mapsto \eta\left(Z_{\delta(\mathbf{d})}\right)
$$

is a one-to-one correspondence from the set $R_{S}^{+}$of Schur roots of the Ringel form $\langle-,-\rangle$in $\mathbb{Z}^{10}$ to the set $\operatorname{ind}(\operatorname{Irr}(\Lambda))-\left\{C_{1}, \ldots, C_{5}\right\}$.

(b) The map

$$
\mathbf{d} \mapsto \psi(\delta(\mathbf{d}))
$$

is a one-to-one correspondence from $R_{S}^{+}$to $\operatorname{ind}(\mathbf{M})-\left\{\mathfrak{p}_{1}, \ldots, \mathfrak{p}_{5}\right\}$.

Note that the descriptions of $R_{S}^{+}, \delta, \eta$ and $\psi$ are completely explicit, so that we get a very concrete parametrization of the factors arising in the canonical factorization of the elements of $\mathcal{S}^{*}$.

Example 3.-(i) Let

$$
\mathbf{d}=\left(\begin{array}{ccc}
1 & 0 \\
2 & 3 & 1 \\
3 & 3 & 3 \\
1 & 2 & 1
\end{array}\right) \in R^{++}
$$

Then $\delta(\mathbf{d})=\mathbf{d}$ and

$$
\psi(\delta(\mathbf{d}))=[1,1]+[1,2]+[1,3]+2[2,3]+[3,4]+[3,5]+[4,4]+[5,5] .
$$

(ii) Let

$$
\mathbf{d}=\left(\begin{array}{cccc}
-1 & 0 \\
0 & & 0 & 0 \\
& 1 & 0 & 0 \\
0 & & 0 & 0
\end{array}\right) \in R^{0}
$$

Then

$$
\delta(\mathbf{d})=\left(\begin{array}{cccc}
0 & 0 & \\
1 & 1 & 0 \\
2 & 2 & 1 \\
0 & 1 & 1 \\
0 & 1 & 1
\end{array}\right)
$$

$4^{\mathrm{e}}$ SÉRIE - TOME $38-2005-\mathrm{N}^{\circ} 2$ 
and

$$
\psi(\delta(\mathbf{d}))=[1,1]+[2,2]+[2,3]+[3,4]+[4,5] .
$$

\section{Case $\mathbb{A}_{5}$ : the component graph}

Recall that the component graph $\mathcal{C}(\Lambda)$ has for vertices the indecomposable irreducible components of the varieties of $\Lambda$-modules, and two vertices $Z_{1}$ and $Z_{2}$ are connected by an edge if and only if $\overline{Z_{1} \oplus Z_{2}}$ is an irreducible component, or equivalently $\operatorname{ext}_{\Lambda}^{1}\left(Z_{1}, Z_{2}\right)=0$. There are edges from the irreducible components $C_{i}(1 \leqslant i \leqslant 5)$ to every other vertex. The following theorem describes all remaining edges. In agreement with Theorem 16.3(a), we shall label the vertices other than $C_{i}$ by the elements of $R_{S}^{+}$.

In order to state the theorem we introduce the following definition. We call a pair of Schur roots $(\mathbf{d}, \mathbf{e})$ critical if the following three conditions hold:

- $\{\mathbf{d}, \mathbf{e}\} \subset R_{\ell}^{\mu}(6)$ for some $\mu \in \mathbb{Q}_{\infty}$ and $\ell \in \mathbb{Z}^{+}$,

$-\langle\mathbf{d}, \mathbf{e}\rangle=0=\langle\mathbf{e}, \mathbf{d}\rangle$,

$-\mathrm{ql}(\mathbf{d})+\mathrm{ql}(\mathbf{e}) \geqslant 7$.

THEOREM 17.1. - Two Schur roots $\mathbf{d}$ and $\mathbf{e}$ are connected by an edge in $\mathcal{C}(\Lambda)$ if and only if the following two conditions hold:

(i) $\langle\mathbf{d}, \mathbf{e}\rangle \geqslant 0$ and $\langle\mathbf{e}, \mathbf{d}\rangle \geqslant 0$,

(ii) $(\mathbf{d}, \mathbf{e})$ is not critical, or $\left\langle\mathbf{d}, \Phi^{i}(\mathbf{e})\right\rangle<0$ where $i=\min \left\{j \geqslant 1 \mid\left\langle\mathbf{d}, \Phi^{j}(\mathbf{e})\right\rangle \neq 0\right\}$.

Proof. - This follows from an adaptation of [24, Theorem 1.3, Lemma 6.4].

Thus, the edges of the component graph of $\Lambda$ are completely determined by the bilinear form $\langle-,-\rangle$ and the Coxeter matrix $\Phi$. Moreover there is an edge between $d$ and $e$ if and only if there is an edge between $\Phi(\mathbf{d})$ and $\Phi(\mathbf{e})$.

\section{Proof of Theorem 10.3}

It was already proved in [24] that $\theta$ and $\eta$ are well-defined and bijective. The map $\phi$ is bijective by definition. It remains to explicitly construct the map $\psi=\phi^{-1} \eta \theta$. It is enough to prove Theorem 10.3 for $\Lambda=\Lambda_{5}$, since $\Lambda_{n}(n=2,3,4)$ are full convex subalgebras of $\Lambda_{5}$.

We will use the following result from [5, Theorem 1,2]: Let $A$ be a tame quasi-tilted basic algebra, and let $\mathbf{d}$ be a dimension vector of an indecomposable $A$-module. Then $\bmod (A, \mathbf{d})$ has at most two irreducible components, and $\bmod (A, \mathbf{d})$ is irreducible if and only if $\mathbf{d}$ is not of one of the following forms:

(a) $\mathbf{d}=\mathbf{h}+\mathbf{z}$ where $\mathbf{h}$ and $\mathbf{z}$ are connected positive vectors with disjoint support, $\langle\mathbf{h}, \mathbf{h}\rangle_{A}=0$, $\langle\mathbf{z}, \mathbf{z}\rangle_{A}=1$ and $z_{i} \leqslant 1$ for all entries $z_{i}$ of $\mathbf{z}$,

(b) $\mathbf{d}=\mathbf{h}+\mathbf{h}^{\prime}$ where $\mathbf{h}$ and $\mathbf{h}^{\prime}$ are connected positive vectors with $\langle\mathbf{h}, \mathbf{h}\rangle_{A}=\left\langle\mathbf{h}^{\prime}, \mathbf{h}^{\prime}\right\rangle_{A}=0$, $\left\langle\mathbf{h}, \mathbf{h}^{\prime}\right\rangle_{A}=1$ and $\left\langle\mathbf{h}^{\prime}, \mathbf{h}\right\rangle_{A}=0$.

The algebras $\Delta=\Delta^{[0,1]}$ and $\Delta^{*}=\Delta^{[-1,0]}$ are both tubular algebras, in particular they are tame quasi-tilted algebras. All connected positive vectors $\mathbf{h}$ for $\Delta^{[i, i+1]}$ such that $\langle\mathbf{h}, \mathbf{h}\rangle^{[i, i+1]}=0$ are of the form $a \mathbf{h}^{[i]}+b \mathbf{h}^{[i+1]}$ where $(0,0) \neq(a, b) \in \mathbb{N} \times \mathbb{N}$. We have $\left\langle\mathbf{h}^{[i]}, \mathbf{h}^{[i+1]}\right\rangle^{[i, i+1]}=6$ and $\left\langle\mathbf{h}^{[i+1]}, \mathbf{h}^{[i]}\right\rangle^{[i, i+1]}=-6$. It follows that the case $(b)$ above cannot occur for $A=\Delta^{[i, i+1]}$, $i=-1,0$. 
For $A=\Delta$ we are in case $(a)$ precisely when $\mathbf{d} \in\left\{\mathbf{e}_{1}(n), \ldots, \mathbf{e}_{5}(n) \mid n \geqslant 1\right\}$ where

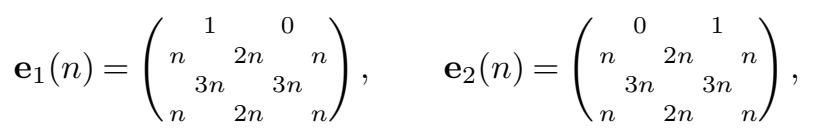

and

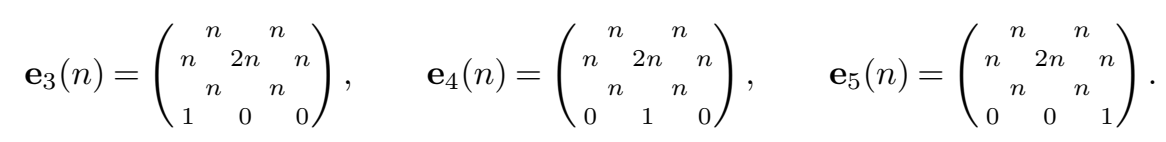

For $A=\Delta^{*}$ we are in case $(a)$ precisely when $\mathbf{d} \in\left\{\left(\mathbf{e}_{1}(n)\right)^{*}, \ldots,\left(\mathbf{e}_{5}(n)\right)^{*} \mid n \geqslant 1\right\}$ where

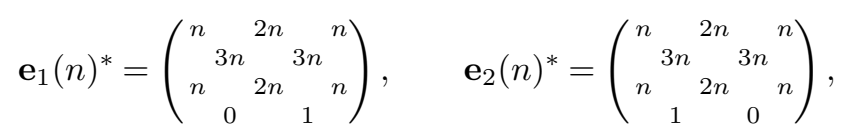

and

$$
\mathbf{e}_{3}(n)^{*}=\left(\begin{array}{ccc}
0 & 0 & 1 \\
n & & n \\
n & 2 n & n \\
n & n
\end{array}\right), \quad \mathbf{e}_{4}(n)^{*}=\left(\begin{array}{ccc}
0 & 1 & 0 \\
n & & n \\
n & 2 n & n \\
n & n
\end{array}\right), \quad \mathbf{e}_{5}(n)^{*}=\left(\begin{array}{ccc}
1 & 0 & 0 \\
n & & n \\
n & 2 n & n \\
n & & n
\end{array}\right) .
$$

Let $M$ be an indecomposable $\widetilde{\Lambda}$-module, and let $\operatorname{dim}(M)$ be its dimension vector. If

$$
\operatorname{dim}(M) \in\left\{\mathbf{e}_{i}(n),\left(\mathbf{e}_{i}(n)\right)^{*} \mid i=1,2, n \geqslant 1\right\},
$$

then $F(M) \in \mathcal{T}_{0}$, and if

$$
\operatorname{dim}(M) \in\left\{\mathbf{e}_{i}(n),\left(\mathbf{e}_{i}(n)\right)^{*} \mid i=3,4,5, n \geqslant 1\right\},
$$

then $F(M) \in \mathcal{T}_{\infty}$. One easily checks that $\mathbf{e}_{i}(n)$ is a Schur root if and only if $n=1$. Set $\mathbf{e}_{i}=\mathbf{e}_{i}(1)$ and $\mathbf{e}_{i}^{*}=\left(\mathbf{e}_{i}(1)\right)^{*}, 1 \leqslant i \leqslant 5$.

Assume that $\mathbf{d} \in I_{S}(\widetilde{\Lambda})$ is a Schur root whose support lies (up to shift) in $\Delta$ or $\Delta^{*}$, and assume $\mathbf{d} \notin\left\{\mathbf{e}_{i}, \mathbf{e}_{i}^{*} \mid 1 \leqslant i \leqslant 5\right\}$. By the result mentioned above this implies that $\bmod (\widetilde{\Lambda}, \mathbf{d})$ is irreducible. Thus $Z_{\mathbf{d}}=Z_{\max }(\mathbf{d})$ and

$$
\psi(\mathbf{d})=\mu\left(Z_{\mathbf{d}}\right)=\mathfrak{m}_{\max }(\mathbf{d}) .
$$

Next, assume $\mathbf{d} \in\left\{\mathbf{e}_{i}, \mathbf{e}_{i}^{*} \mid 1 \leqslant i \leqslant 5\right\}$. Thus $\bmod (\widetilde{\Lambda}, \mathbf{d})$ has exactly two irreducible components. Furthermore, we know that

$$
Z_{\mathbf{d}}=\overline{\mathcal{O}\left(M_{\mathbf{d}}\right)}
$$

for some indecomposable $\widetilde{\Lambda}$-module $M_{\mathbf{d}}$.

For any vertex $i_{j}$ of $\widetilde{Q}$ let $S_{i_{j}}$ be the corresponding simple $\widetilde{\Lambda}$-module, and let $Z_{i_{j}}$ be the irreducible component consisting of the single point corresponding to $S_{i_{j}}$. Then

$$
\operatorname{ext}_{\widetilde{\Lambda}}^{1}\left(Z_{\mathbf{h}_{0}}, Z_{i_{j}}\right)=\operatorname{ext}_{\widetilde{\Lambda}}^{1}\left(Z_{i_{j}}, Z_{\mathbf{h}_{0}}\right)=0
$$

$4^{\mathrm{e}}$ SÉRIE - TOME $38-2005-\mathrm{N}^{\circ} 2$ 
for $i=2,4$ and all $j \in \mathbb{Z}$, and

$$
\operatorname{ext}_{\widetilde{\Lambda}}^{1}\left(Z_{\mathbf{h}_{\infty}}, Z_{i_{j}}\right)=\operatorname{ext}_{\widetilde{\Lambda}}^{1}\left(Z_{i_{j}}, Z_{\mathbf{h}_{\infty}}\right)=0
$$

for $i=1,3,5$ and all $j \in \mathbb{Z}$. Thus [14, Theorem 1.2] implies that $\overline{Z_{\mathbf{h}_{0}} \oplus Z_{i_{j}}}$ (resp. $\overline{Z_{\mathbf{h}_{\infty}} \oplus Z_{i_{j}}}$ ) are irreducible components provided $i=2,4$ (resp. $i=1,3,5$ ). Exactly one of these irreducible components lies in $\bmod (\widetilde{\Lambda}, \mathbf{d})$, we denote this component by $Z_{\mathrm{dec}}(\mathbf{d})$. Thus $\bmod (\widetilde{\Lambda}, \mathbf{d})$ contains exactly one indecomposable irreducible component, namely $Z_{\mathbf{d}}$, and exactly one decomposable irreducible component, namely $Z_{\mathrm{dec}}(\mathbf{d})$.

If $\overline{Z^{\prime} \oplus Z^{\prime \prime}}$ is an irreducible component, then

$$
\mu\left(\overline{Z^{\prime} \oplus Z^{\prime \prime}}\right)=\mu\left(Z^{\prime}\right)+\mu\left(Z^{\prime \prime}\right) .
$$

Thus, if $\mathbf{d} \notin\left\{\mathbf{e}_{3}^{*}, \mathbf{e}_{5}\right\}$, then $Z_{\mathrm{dec}}(\mathbf{d}) \neq Z_{\max }(\mathbf{d})$. This yields $Z_{\mathbf{d}}=Z_{\max }(\mathbf{d})$ and

$$
\psi(\mathbf{d})=\mu\left(Z_{\mathbf{d}}\right)=\mathfrak{m}_{\max }(\mathbf{d}) .
$$

For $\mathbf{d} \in\left\{\mathbf{e}_{3}^{*}, \mathbf{e}_{5}\right\}$ it is not difficult to construct the module $M_{\mathbf{d}}$ explicitly. We get

$$
\psi\left(\mathbf{e}_{3}^{*}\right)=2[1,1]+[2,2]+[2,4]+[3,3]+[4,5]
$$

and

$$
\psi\left(\mathbf{e}_{5}\right)=[1,2]+[2,4]+[3,3]+[4,4]+2[5,5] .
$$

The only Schur roots in $I_{S}(\widetilde{\Lambda})$ whose support is (up to shift) not contained in $\Delta$ or $\Delta^{*}$ are

$$
\mathbf{f}_{1}=\left(\begin{array}{ccc}
0 & 1 \\
1 & 2 & 1 \\
3 & 2 & 2 \\
1 & 2 & 1 \\
0 & 1
\end{array}\right), \quad \mathbf{f}_{2}=\left(\begin{array}{ccc}
1 & 0 \\
1 & 2 & 1 \\
2 & 3 & 3 \\
1 & 2 & 1 \\
1 & 0
\end{array}\right), \quad \mathbf{p}_{2}, \quad \mathbf{p}_{4}
$$

(these are all in $\mathcal{T}_{0}$ ), and

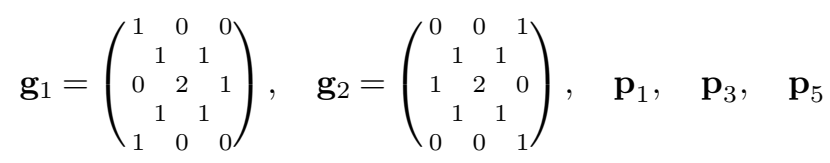

(these are all in $\mathcal{T}_{\infty}$ ). Here $\mathbf{p}_{1}, \ldots, \mathbf{p}_{5}$ are (up to shift) the dimension vectors of the indecomposable projective $\widetilde{\Lambda}$-modules as displayed in 15.3 .

For each $\mathbf{d} \in\left\{\mathbf{p}_{1}, \ldots, \mathbf{p}_{5}, \mathbf{f}_{1}, \mathbf{f}_{2}, \mathbf{g}_{1}, \mathbf{g}_{2}\right\}$, there exists an indecomposable $\widetilde{\Lambda}$-module $M_{\mathbf{d}}$ such that $Z_{\mathbf{d}}=\overline{\mathcal{O}\left(M_{\mathbf{d}}\right)}$. For $\mathbf{d} \in\left\{\mathbf{p}_{1}, \ldots, \mathbf{p}_{5}, \mathbf{g}_{1}, \mathbf{g}_{2}\right\}$ it is easy to construct $M_{\mathbf{d}}$ explicitly. In these cases, it follows that $Z_{\mathbf{d}}=Z_{\max }(\mathbf{d})$, thus

$$
\psi(\mathbf{d})=\mu\left(Z_{\mathbf{d}}\right)=\mathfrak{m}_{\max }(\mathbf{d}) .
$$

For any element $a \in \widetilde{\Lambda}$ and any $\widetilde{\Lambda}$-module $M$, let $f_{a, M}: M \rightarrow M$ be the linear map defined by the $\widetilde{\Lambda}$-module action of $a$ on $M$, i.e. $f_{a, M}(m)=a m$. If $M$ is a submodule of a module $N$, then we have

$$
\operatorname{rk}\left(f_{a, M}\right) \geqslant \operatorname{rk}\left(f_{a, N}\right)
$$

ANNALES SCIENTIFIQUES DE L'ÉCOLE NORMALE SUPÉRIEURE 
for all $a$. Now let

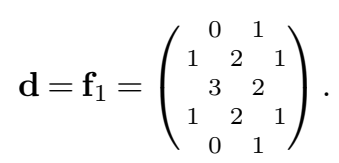

In Section 20.6 we can see that $M_{\mathbf{d}}$ contains two indecomposable submodules $M_{\mathbf{f}_{1}^{\prime}}$ and $M_{\mathbf{f}_{1}^{\prime \prime}}$ with dimension vectors

$$
\mathbf{f}_{1}^{\prime}=\left(\begin{array}{ccc}
0 & 0 \\
1 & 2 & 1 \\
3 & 2 & 2 \\
1 & 2 & 1 \\
0 & 1 & 1
\end{array}\right) \quad \text { and } \quad \mathbf{f}_{1}^{\prime \prime}=\left(\begin{array}{ccc}
0 & 1 \\
0 & 1 & 1 \\
2 & 1 & 1 \\
1 & 1 & 0 \\
0 & 0 & 0
\end{array}\right)
$$

respectively. We get

$$
Z_{\mathbf{f}_{1}^{\prime}}=\overline{\mathcal{O}\left(M_{\mathbf{f}_{1}^{\prime}}\right)} \quad \text { and } \quad Z_{\mathbf{f}_{1}^{\prime \prime}}=\overline{\mathcal{O}\left(M_{\mathbf{f}_{1}^{\prime \prime}}\right)}
$$

Since the support of $\mathbf{f}_{1}^{\prime}$ and $\mathbf{f}_{1}^{\prime \prime}$ lies in $\Delta^{*}$ and $\Delta$, respectively, we get

$$
\psi\left(\mathbf{f}_{1}^{\prime}\right)=\mu\left(Z_{\mathbf{f}_{1}^{\prime}}\right)=\mathfrak{m}_{\max }\left(\mathbf{f}_{1}^{\prime}\right) \quad \text { and } \quad \psi\left(\mathbf{f}_{1}^{\prime \prime}\right)=\mu\left(Z_{\mathbf{f}_{1}^{\prime \prime}}\right)=\mathfrak{m}_{\max }\left(\mathbf{f}_{1}^{\prime \prime}\right) .
$$

This enables us to compute the ranks of the maps $f_{p, M_{\mathrm{f}_{1}^{\prime}}}$ and $f_{p, M_{\mathrm{f}_{1}^{\prime \prime}}}$ for all paths $p$ in $\widetilde{Q}$ of the form $\alpha_{l j} \alpha_{l+1, j} \cdots \alpha_{m j}, 1 \leqslant l \leqslant m \leqslant 4$. Then we can use the above rank inequality, and get the rank of $f_{p, M_{\mathbf{f}_{1}}}$ for any path $p$. It turns out that

$$
\psi\left(\mathbf{f}_{1}\right)=\mu\left(Z_{\mathbf{f}_{1}}\right)=\mathfrak{m}_{\max }\left(\mathbf{f}_{1}\right) .
$$

The case $\mathbf{d}=\mathbf{f}_{2}$ is done in a similar way, and we get again

$$
\psi\left(\mathbf{f}_{2}\right)=\mu\left(Z_{\mathbf{f}_{2}}\right)=\mathfrak{m}_{\max }\left(\mathbf{f}_{2}\right) .
$$

\section{Concluding remarks}

19.1. By Proposition 3.3, the preprojective algebra $\Lambda=P(Q)$ is tame if and only if the quiver $Q$ is of Dynkin type $\mathbb{A}_{5}$ or $\mathbb{D}_{4}$. Using the same methods as in this paper it is possible to obtain a complete analogue of Theorem 1.3 for type $\mathbb{D}_{4}$. In this case, $\Lambda$ has a Galois covering which is isomorphic to the repetitive algebra of a tubular algebra of type $(3,3,3)$, and the corresponding root system is an elliptic root system of type $\mathbb{E}_{6}^{(1,1)}$. We plan to give a detailed analysis of this case in a forthcoming publication.

19.2. It is shown in [3] that for all Dynkin types, the algebra $\mathbb{C}[N]$ has the structure of an (upper) cluster algebra, and that it has finite type as a cluster algebra if and only $\mathfrak{g}$ is of Lie type $\mathbb{A}_{n}(n \leqslant 4)$. In that case one can associate to $\mathbb{C}[N]$ a root system $\mathcal{R}$ called its cluster type, which controls the combinatorics of the cluster variables and of the cluster sets. More precisely, the cluster variables are parametrized by the set $\mathcal{R} \geqslant-1$ of almost positive roots of $\mathcal{R}$, and the pairs of cluster variables which can occur simultaneously in a cluster set can be explicitly described in terms of $\mathcal{R}$ and a piecewise linear Coxeter transformation acting on $\mathcal{R}_{\geqslant-1}$. The Cartan matrix $A$ of $\mathcal{R}$ can be obtained by a certain symmetrization procedure from the principal part $B(t)$ of the exchange matrix of $\mathbb{C}[N]$ at certain vertices $t$ of its exchange graph (see [20]).

For $\mathfrak{g}$ of Lie type $\mathbb{A}_{2}, \mathbb{A}_{3}, \mathbb{A}_{4}$, the algebra $\mathbb{C}[N]$ has cluster type $\mathbb{A}_{1}, \mathbb{A}_{3}, \mathbb{D}_{6}$ respectively [3]. As mentioned in 9.10 , these root systems also occur in our setting in the following way. For

$4^{\mathrm{e}}$ SÉRIE - TOME $38-2005-\mathrm{N}^{\circ} 2$ 
$n=2,3,4$, we have $\underline{\bmod }\left(\widetilde{\Lambda}_{n}\right) \cong D^{b}\left(\bmod \left(\mathbb{C} \mathcal{Q}_{n}\right)\right)$ where $\mathcal{Q}_{n}$ is a quiver of type $\mathbb{A}_{1}, \mathbb{A}_{3}, \mathbb{D}_{6}$ respectively.

19.3. At the moment, there is no notion of cluster type for the algebras $\mathbb{C}[N]$ which are not of finite type as cluster algebras. The results of this paper strongly suggest that if such a cluster type exists for $\mathfrak{g}$ of Lie type $\mathbb{A}_{5}\left(\right.$ resp. $\left.\mathbb{D}_{4}\right)$, then it should be the elliptic type $\mathbb{E}_{8}^{(1,1)}$ (resp. $\mathbb{E}_{6}^{(1,1)}$ ) in the notation of Saito, or the tubular type $(6,3,2)$ (resp. $(3,3,3)$ ) in the language of Ringel. Remember in particular that $\underline{\bmod }\left(\widetilde{\Lambda}_{5}\right) \cong D^{b}(\operatorname{coh}(\mathbb{X}))$ where $\mathbb{X}$ is a weighted projective line of type $(6,3,2)$.

19.4. Here is another remark supporting that guess. For $\mathfrak{g}$ of Lie type $\mathbb{A}_{5}$ one can find a vertex $t$ of the exchange graph of $\mathbb{C}[N]$ at which the principal part of the exchange matrix is (up to simultaneous permutations of rows and columns)

$$
B(t)=\left(\begin{array}{cccccccccc}
0 & 0 & 1 & 0 & 0 & 0 & 0 & 0 & 0 & 0 \\
0 & 0 & 0 & 1 & 0 & 0 & 0 & 0 & 0 & -1 \\
-1 & 0 & 0 & 1 & 0 & 0 & 0 & 0 & 0 & -1 \\
0 & -1 & -1 & 0 & -1 & 0 & 0 & 0 & 0 & 2 \\
0 & 0 & 0 & 1 & 0 & -1 & 0 & 0 & 0 & -1 \\
0 & 0 & 0 & 0 & 1 & 0 & -1 & 0 & 0 & 0 \\
0 & 0 & 0 & 0 & 0 & 1 & 0 & -1 & 0 & 0 \\
0 & 0 & 0 & 0 & 0 & 0 & 1 & 0 & -1 & 0 \\
0 & 0 & 0 & 0 & 0 & 0 & 0 & 1 & 0 & 0 \\
0 & 1 & 1 & -2 & 1 & 0 & 0 & 0 & 0 & 0
\end{array}\right) .
$$

We suggest to take as symmetric counterpart of $B(t)$ the matrix

$$
A=\left(\begin{array}{cccccccccc}
2 & 0 & -1 & 0 & 0 & 0 & 0 & 0 & 0 & 0 \\
0 & 2 & 0 & -1 & 0 & 0 & 0 & 0 & 0 & -1 \\
-1 & 0 & 2 & -1 & 0 & 0 & 0 & 0 & 0 & -1 \\
0 & -1 & -1 & 2 & -1 & 0 & 0 & 0 & 0 & 2 \\
0 & 0 & 0 & -1 & 2 & -1 & 0 & 0 & 0 & -1 \\
0 & 0 & 0 & 0 & -1 & 2 & -1 & 0 & 0 & 0 \\
0 & 0 & 0 & 0 & 0 & -1 & 2 & -1 & 0 & 0 \\
0 & 0 & 0 & 0 & 0 & 0 & -1 & 2 & -1 & 0 \\
0 & 0 & 0 & 0 & 0 & 0 & 0 & -1 & 2 & 0 \\
0 & -1 & -1 & 2 & -1 & 0 & 0 & 0 & 0 & 2
\end{array}\right) .
$$

Note that $A$ has two positive entries off the diagonal, so it differs from the matrix obtained from $B(t)$ by the symmetrizing procedure of [20]. It turns out that $A$ is exactly the Cartan matrix of

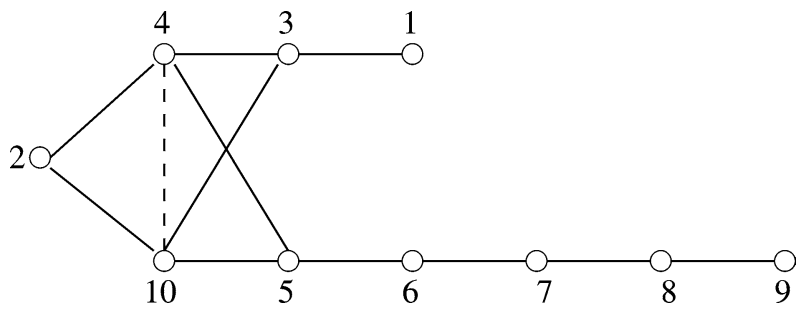

Fig. 2. The Dynkin diagram of $\mathbb{E}_{8}^{(1,1)}$. 
the root system $\mathbb{E}_{8}^{(1,1)}$, that is, the matrix of scalar products of a basis of simple roots in the sense of Saito [50]. It can be visualized with the help of the Dynkin type diagram of Fig. 2 in which an ordinary edge between $i$ and $j$ means that $a_{i j}=a_{j i}=-1$ and the dashed line between 4 and 10 means that $a_{4,10}=a_{10,4}=2$ (see [50]).

There is a similar fact for type $\mathbb{D}_{4}$ and $\mathbb{E}_{6}^{(1,1)}$.

\section{Pictures and tables}

20.1. The Auslander-Reiten quivers of $\Lambda_{2}, \Lambda_{3}, \Lambda_{4}$

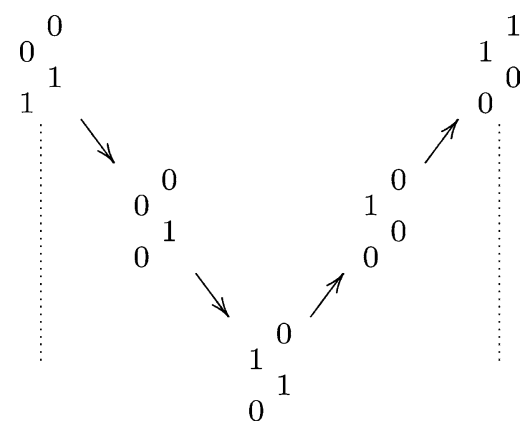

The Auslander-Reiten quiver of $\Lambda_{2}$

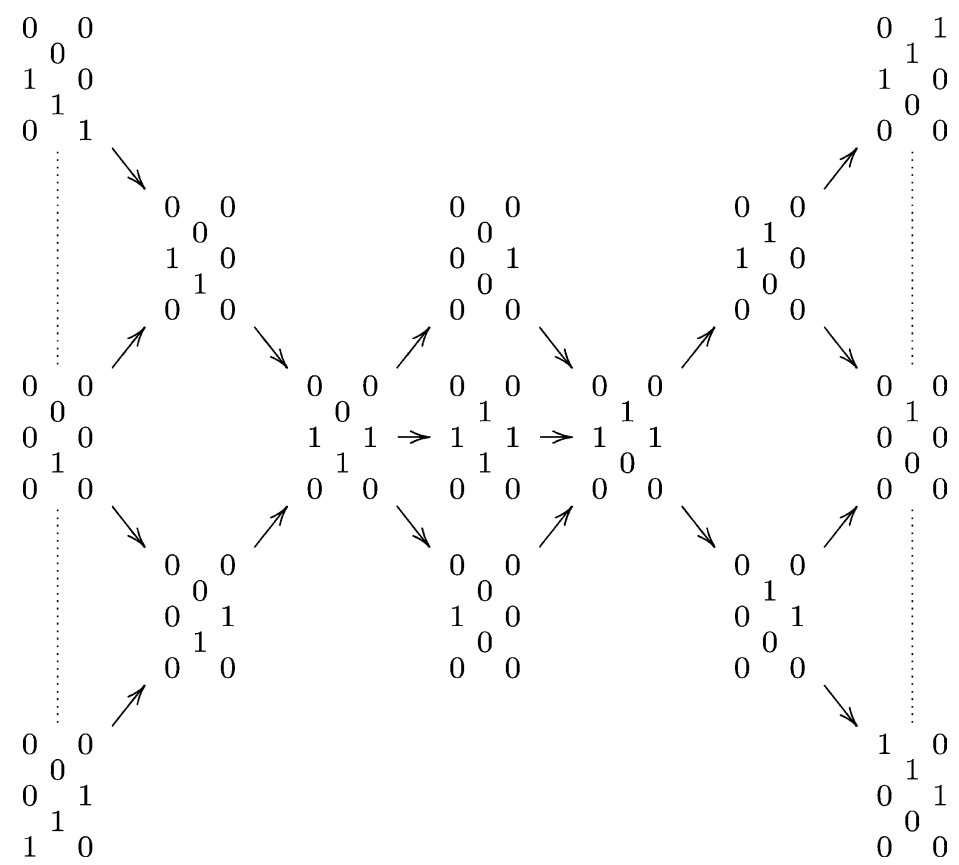

The Auslander-Reiten quiver of $\Lambda_{3}$ 


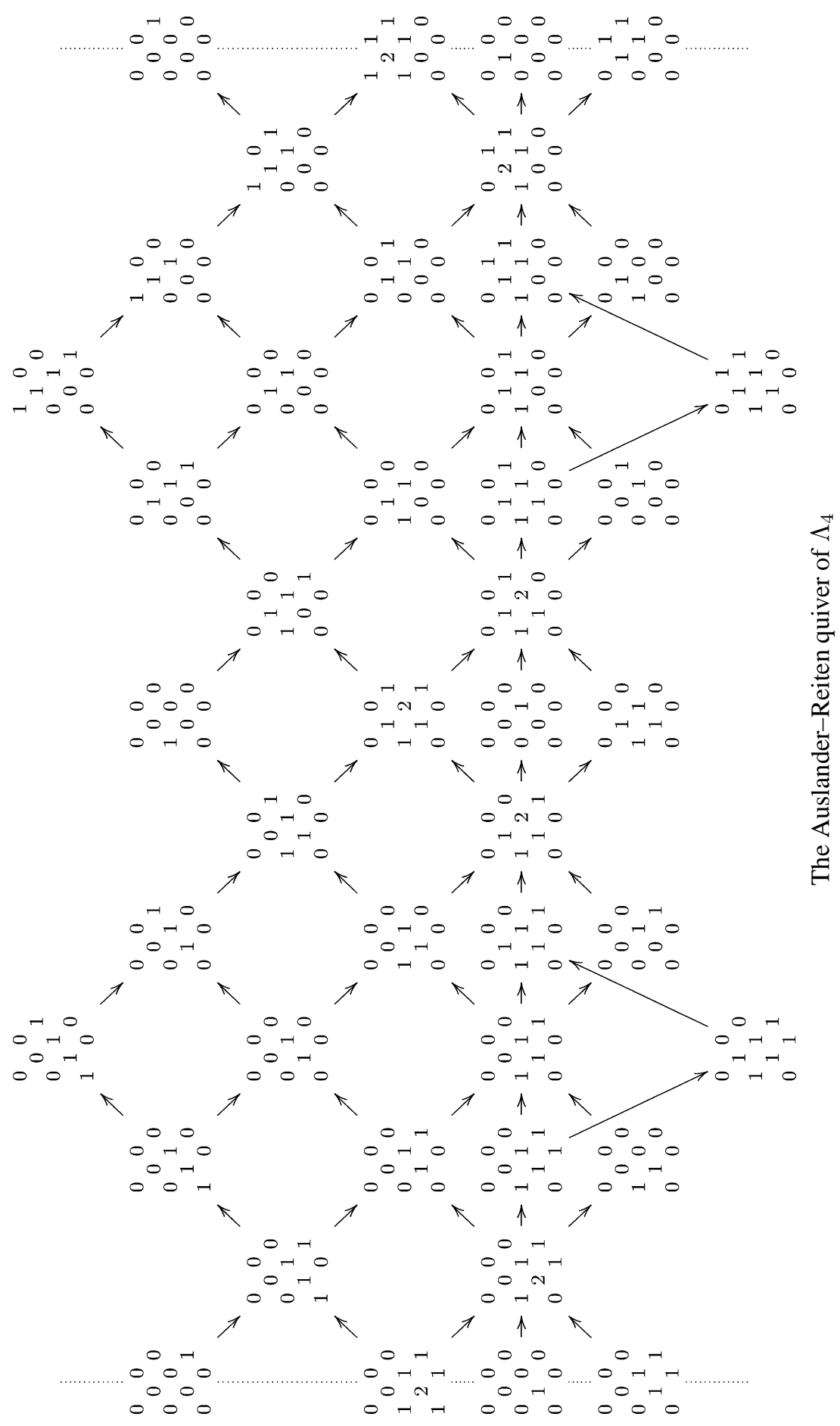




\subsection{The indecomposable multisegments in $\mathbf{M}(4)$}

$$
\begin{aligned}
& \mathfrak{m}_{1}=[1,1] \\
& \mathfrak{m}_{21}=[1,3]+[4,4] \\
& \mathfrak{m}_{2}=[2,2] \\
& \mathfrak{m}_{22}=[1,2]+[3,3]+[4,4] \\
& \mathfrak{m}_{3}=[3,3] \\
& \mathfrak{m}_{23}=[1,1]+[2,2]+[3,4] \\
& \mathfrak{m}_{4}=[4,4] \\
& \mathfrak{m}_{24}=[1,1]+[2,4] \\
& \mathfrak{m}_{5}=[1,2] \\
& \mathfrak{m}_{25}=[1,2]+[3,4] \\
& \mathfrak{m}_{6}=[1,1]+[2,2] \\
& \mathfrak{m}_{26}=[1,1]+[2,3]+[4,4] \\
& \mathfrak{m}_{7}=[2,3] \\
& \mathfrak{m}_{27}=[1,2]+[2,3]+[4,4] \\
& \mathfrak{m}_{8}=[2,2]+[3,3] \\
& \mathfrak{m}_{28}=[1,2]+[2,4] \\
& \mathfrak{m}_{9}=[3,4] \\
& \mathfrak{m}_{29}=[1,3]+[3,4] \\
& \mathfrak{m}_{10}=[3,3]+[4,4] \\
& \mathfrak{m}_{30}=[1,1]+[2,3]+[3,4] \\
& \mathfrak{m}_{11}=[1,3] \\
& \mathfrak{m}_{31}=[1,2]+[2,3]+[3,3]+[4,4] \\
& \mathfrak{m}_{12}=[1,1]+[2,2]+[3,3] \\
& \mathfrak{m}_{32}=[1,2]+[2,4]+[3,3] \\
& \mathfrak{m}_{13}=[1,2]+[3,3] \\
& \mathfrak{m}_{33}=[1,3]+[2,2]+[3,4] \\
& \mathfrak{m}_{14}=[1,1]+[2,3] \\
& \mathfrak{m}_{34}=[1,1]+[2,2]+[2,3]+[3,4] \\
& \mathfrak{m}_{15}=[1,2]+[2,3] \\
& \mathfrak{m}_{35}=[1,1]+[1,3]+[2,2]+[3,4] \\
& \mathfrak{m}_{16}=[2,4] \\
& \mathfrak{m}_{36}=[1,2]+[2,4]+[3,3]+[4,4] \\
& \mathfrak{m}_{17}=[2,2]+[3,3]+[4,4] \\
& \mathfrak{m}_{37}=[1,4] \\
& \mathfrak{m}_{18}=[2,3]+[4,4] \\
& \mathfrak{m}_{38}=[1,3]+[2,4] \\
& \mathfrak{m}_{19}=[2,2]+[3,4] \\
& \mathfrak{m}_{39}=[1,2]+[2,3]+[3,4] \\
& \mathfrak{m}_{20}=[2,3]+[3,4] \\
& \mathfrak{m}_{40}=[1,1]+[2,2]+[3,3]+[4,4]
\end{aligned}
$$

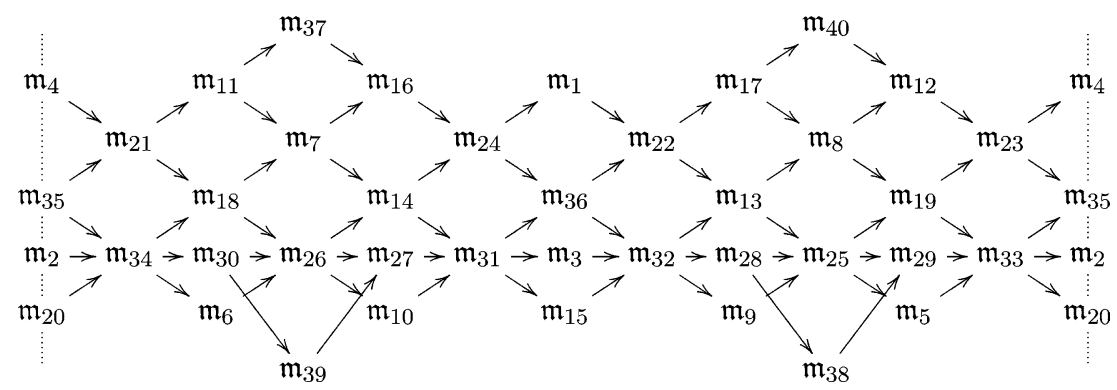

The Auslander-Reiten quiver of $\Lambda_{4}$ in terms of multisegments 
20.3. Extensions between indecomposable $\Lambda_{3}$-modules
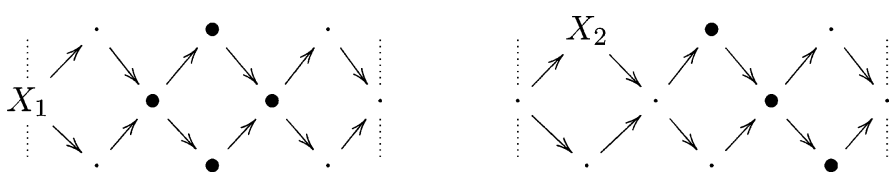

20.4. Extensions between indecomposable $\Lambda_{4}$-modules
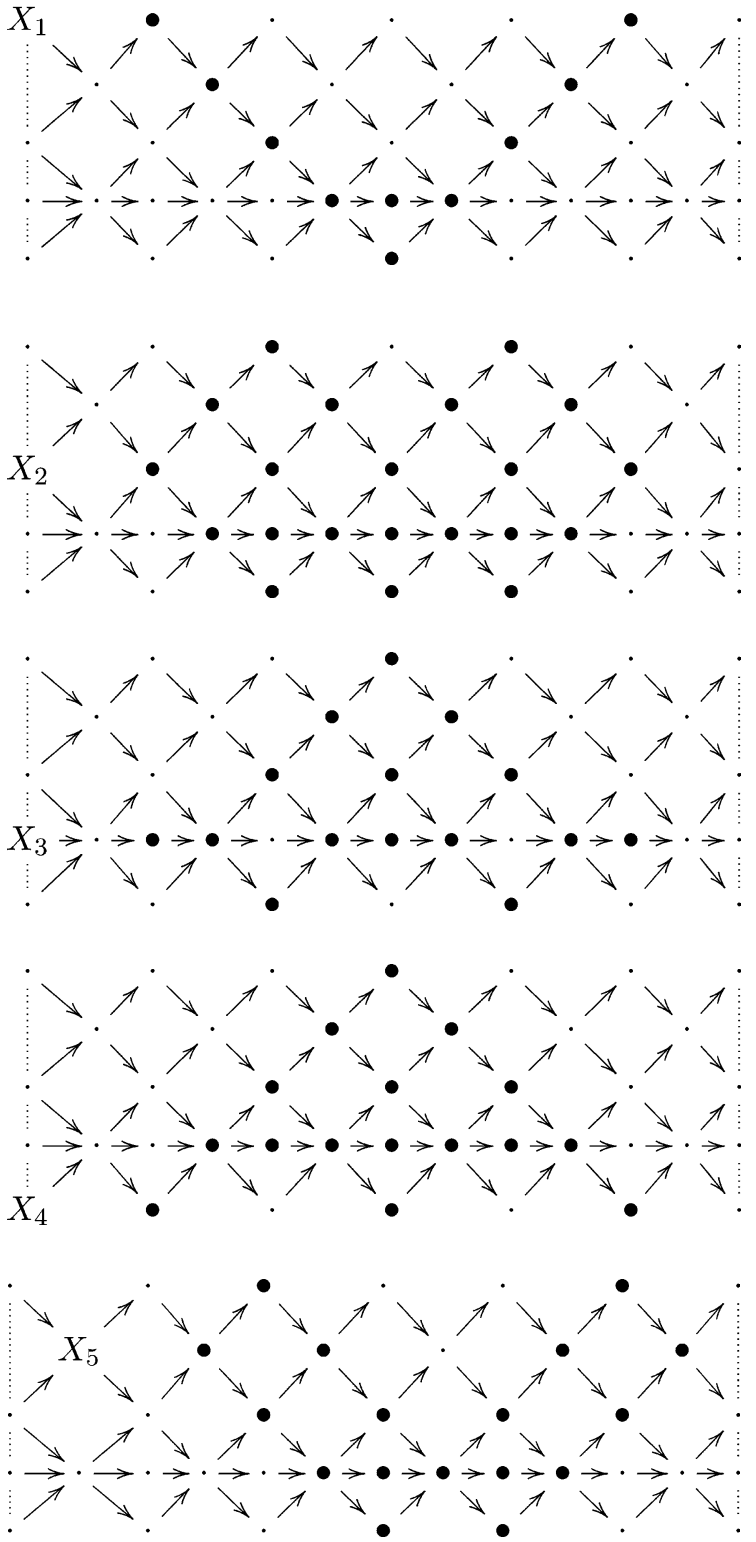


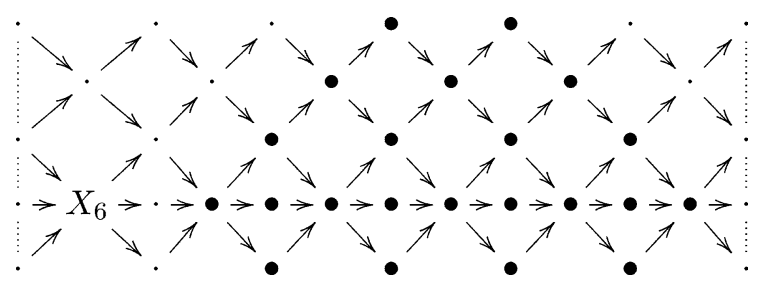

20.5. The graph $\mathcal{G}_{4}^{\circ}$

\begin{tabular}{|c|c|c|c|c|c|c|c|c|c|}
\hline$(1,3)$ & $(1,4)$ & $(1,5)$ & $(1,6)$ & $(1,9)$ & $(1,10)$ & $(1,11)$ & $(1,12)$ & $(1,13)$ & $(1,14)$ \\
\hline$(1,15)$ & $(1,21)$ & $(1,22)$ & $(1,23)$ & $(1,24)$ & $(1,25)$ & $(1,26)$ & $(1,27)$ & $(1,28)$ & $(1,29)$ \\
\hline$(1,30)$ & $(1,31)$ & $(1,32)$ & $(1,35)$ & $(1,36)$ & $(2,4)$ & $(2,5)$ & $(2,6)$ & $(2,7)$ & $(2,8)$ \\
\hline$(2,11)$ & $(2,12)$ & $(2,15)$ & $(2,16)$ & $(2,17)$ & $(2,18)$ & $(2,19)$ & $(2,20)$ & $(2,21)$ & $(2,23)$ \\
\hline$(2,27)$ & $(2,28)$ & $(2,33)$ & $(2,34)$ & $(2,35)$ & $(3,7)$ & $(3,8)$ & $(3,9)$ & $(3,10)$ & $(3,11)$ \\
\hline$(3,12)$ & $(3,13)$ & $(3,14)$ & $(3,15)$ & $(3,16)$ & $(3,17)$ & $(3,20)$ & $(3,22)$ & $(3,24)$ & $(3,29)$ \\
\hline$(3,30)$ & $(3,31)$ & $(3,32)$ & $(3,36)$ & $(4,5)$ & $(4,6)$ & $(4,9)$ & $(4,10)$ & $(4,16)$ & $(4,17)$ \\
\hline$(4,18)$ & $(4,19)$ & $(4,20)$ & $(4,21)$ & $(4,22)$ & $(4,23)$ & $(4,24)$ & $(4,25)$ & $(4,26)$ & $(4,27)$ \\
\hline$(4,28)$ & $(4,29)$ & $(4,30)$ & $(4,33)$ & $(4,34)$ & $(4,35)$ & $(4,36)$ & $(5,6)$ & $(5,8)$ & $(5,11)$ \\
\hline$(5,12)$ & $(5,13)$ & $(5,15)$ & $(5,17)$ & $(5,19)$ & $(5,21)$ & $(5,22)$ & $(5,23)$ & $(5,25)$ & $(5,27)$ \\
\hline$(5,28)$ & $(5,29)$ & $(5,33)$ & $(5,35)$ & $(6,7)$ & $(6,11)$ & $(6,12)$ & $(6,14)$ & $(6,15)$ & $(6,16)$ \\
\hline$(6,18)$ & $(6,21)$ & $(6,23)$ & $(6,24)$ & $(6,26)$ & $(6,27)$ & $(6,28)$ & $(6,30)$ & $(6,34)$ & $(6,35)$ \\
\hline$(7,8)$ & $(7,10)$ & $(7,11)$ & $(7,12)$ & $(7,14)$ & $(7,15)$ & $(7,16)$ & $(7,17)$ & $(7,18)$ & $(7,20)$ \\
\hline$(7,26)$ & $(7,27)$ & $(7,30)$ & $(7,31)$ & $(7,34)$ & $(8,9)$ & $(8,11)$ & $(8,12)$ & $(8,13)$ & $(8,15)$ \\
\hline$(8,16)$ & $(8,17)$ & $(8,19)$ & $(8,20)$ & $(8,25)$ & $(8,28)$ & $(8,29)$ & $(8,32)$ & $(8,33)$ & $(9,10)$ \\
\hline$(9,12)$ & $(9,13)$ & $(9,16)$ & $(9,17)$ & $(9,19)$ & $(9,20)$ & $(9,22)$ & $(9,23)$ & $(9,24)$ & $(9,25)$ \\
\hline$(9,28)$ & $(9,29)$ & $(9,30)$ & $(9,32)$ & $(9,36)$ & $(10,11)$ & $(10,14)$ & $(10,16)$ & $(10,17)$ & $(10,18)$ \\
\hline$(10,20)$ & $(10,21)$ & $(10,22)$ & $(10,24)$ & $(10,26)$ & $(10,27)$ & $(10,29)$ & $(10,30)$ & $(10,31)$ & $(10,36)$ \\
\hline$(11,12)$ & $(11,13)$ & $(11,14)$ & $(11,15)$ & $(11,17)$ & $(11,18)$ & $(11,20)$ & $(11,21)$ & $(11,22)$ & $(11,26)$ \\
\hline$(11,27)$ & $(11,29)$ & $(11,30)$ & $(11,31)$ & $(11,33)$ & $(11,34)$ & $(11,35)$ & $(12,13)$ & $(12,14)$ & $(12,15)$ \\
\hline$(12,16)$ & $(12,19)$ & $(12,20)$ & $(12,23)$ & $(12,24)$ & $(12,25)$ & $(12,28)$ & $(12,29)$ & $(12,30)$ & $(12,32)$ \\
\hline$(12,33)$ & $(12,34)$ & $(12,35)$ & $(13,15)$ & $(13,17)$ & $(13,22)$ & $(13,25)$ & $(13,28)$ & $(13,29)$ & $(13,32)$ \\
\hline$(14,15)$ & $(14,16)$ & $(14,24)$ & $(14,26)$ & $(14,27)$ & $(14,30)$ & $(14,31)$ & $(15,16)$ & $(15,17)$ & $(15,22)$ \\
\hline$(15,24)$ & $(15,27)$ & $(15,28)$ & $(15,31)$ & $(15,32)$ & $(15,36)$ & $(16,17)$ & $(16,18)$ & $(16,19)$ & $(16,20)$ \\
\hline$(16,23)$ & $(16,24)$ & $(16,26)$ & $(16,27)$ & $(16,28)$ & $(16,30)$ & $(16,31)$ & $(16,32)$ & $(16,34)$ & $(16,36)$ \\
\hline$(17,18)$ & $(17,19)$ & $(17,20)$ & $(17,21)$ & $(17,22)$ & $(17,25)$ & $(17,27)$ & $(17,28)$ & $(17,29)$ & $(17,31)$ \\
\hline$(17,32)$ & $(17,33)$ & $(17,36)$ & $(18,20)$ & $(18,21)$ & $(18,26)$ & $(18,27)$ & $(18,30)$ & $(18,34)$ & $(19,20)$ \\
\hline$(19,23)$ & $(19,25)$ & $(19,28)$ & $(19,29)$ & $(19,33)$ & $(20,21)$ & $(20,23)$ & $(20,29)$ & $(20,30)$ & $(20,33)$ \\
\hline
\end{tabular}




$\begin{array}{llllllllll}(20,34) & (20,35) & (21,22) & (21,26) & (21,27) & (21,29) & (21,30) & (21,33) & (21,34) & (21,35) \\ (22,25) & (22,27) & (22,28) & (22,29) & (22,31) & (22,32) & (22,36) & (23,24) & (23,25) & (23,28) \\ (23,29) & (23,30) & (23,33) & (23,34) & (23,35) & (24,26) & (24,27) & (24,28) & (24,30) & (24,31) \\ (24,32) & (24,36) & (25,28) & (25,29) & (26,27) & (26,30) & (27,28) & (27,31) & (27,36) & (28,32) \\ (28,36) & (29,30) & (29,33) & (29,35) & (30,34) & (30,35) & (31,36) & (32,36) & (33,35) & (34,35)\end{array}$

20.6. The non-homogeneous tubes in $\mathcal{T}_{0}$
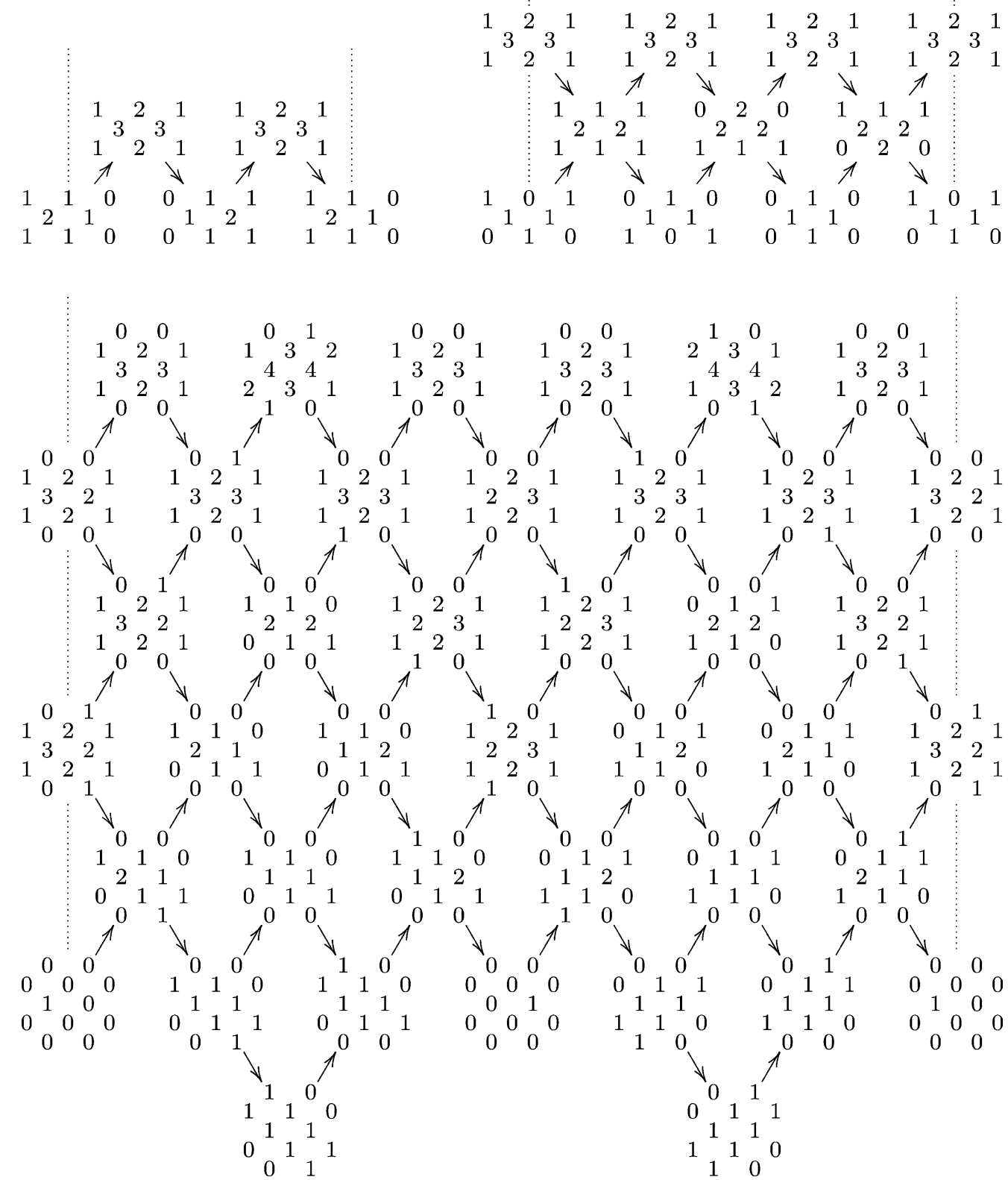

ANNALES SCIENTIFIQUES DE L'ÉCOLE NORMALE SUPÉRIEURE 
20.7. The non-homogeneous tubes in $\mathcal{T}_{\infty}$

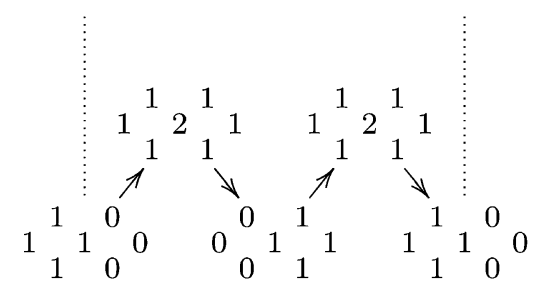

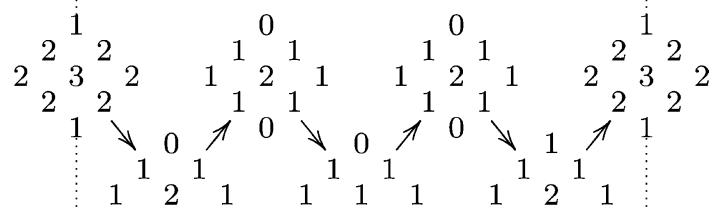

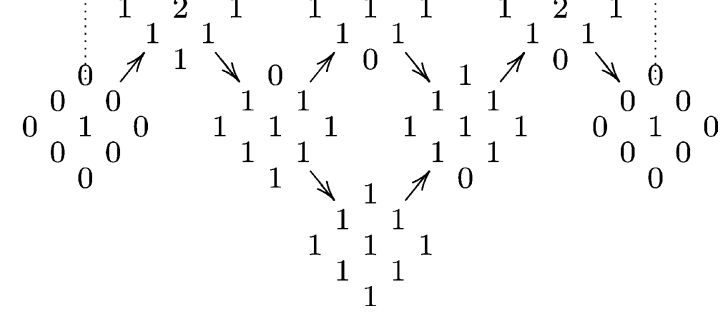

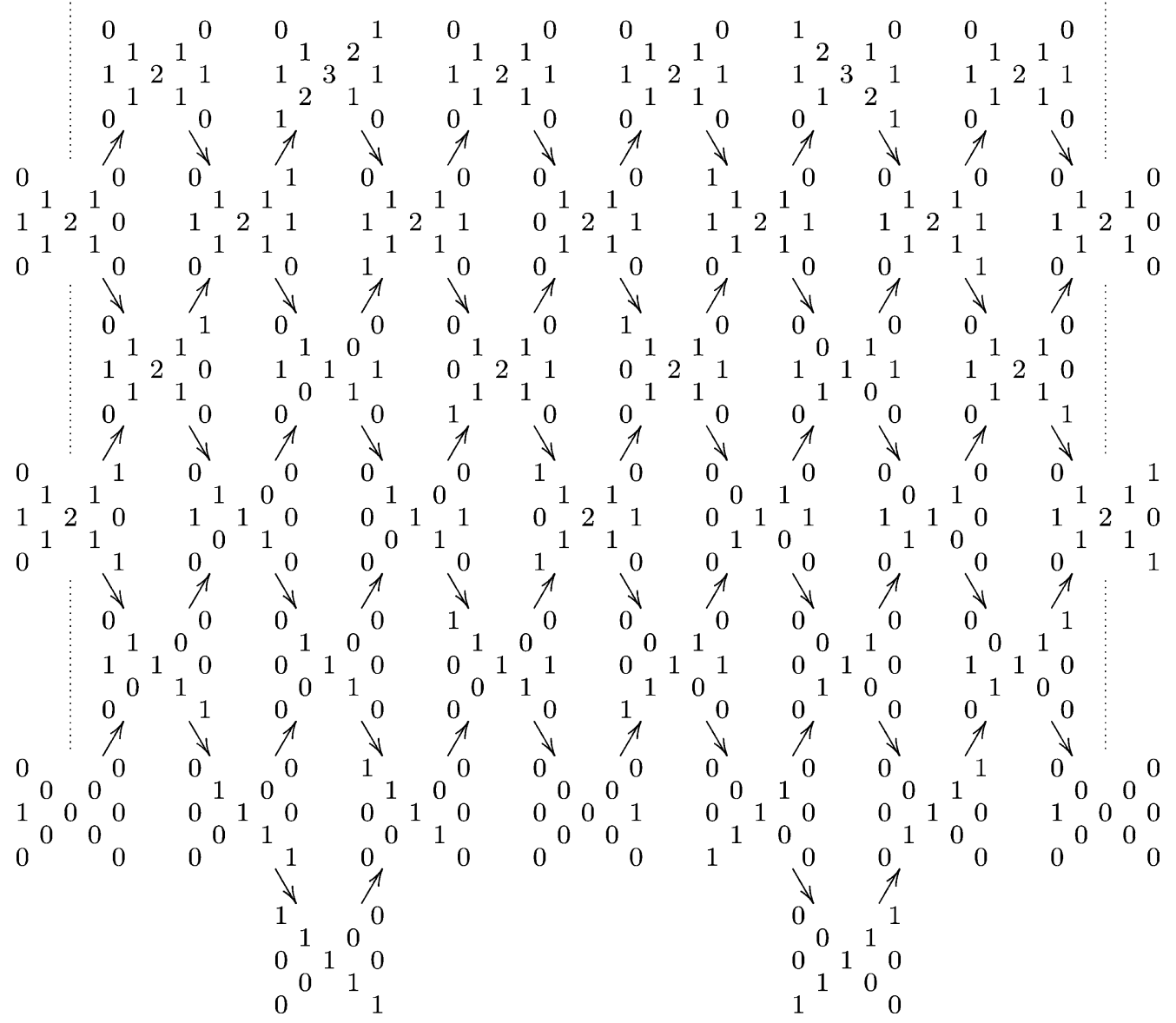

$4^{\mathrm{e}}$ SÉRIE - TOME $38-2005-\mathrm{N}^{\circ} 2$ 
20.8. The sets $R_{[m, n]}(i)$

20.8.1. The sets $R_{[m, n]}(2),[m, n] \in L_{2}$

$$
\begin{aligned}
& {\left[[1,0],\left[\begin{array}{ccc}
0 & 0 \\
0 & 1 & 1 \\
1 & 2 & 2 \\
0 & 1 & 1
\end{array}\right],\left[\begin{array}{ccc}
0 & 0 \\
1 & 1 & 0 \\
2 & 1 \\
1 & 1 & 0
\end{array}\right]\right]} \\
& {\left[[1,1],\left[\begin{array}{ccc}
1 & 0 \\
1 & 2 & 1 \\
2 & 2 \\
0 & 1 & 1
\end{array}\right],\left[\begin{array}{ccc}
0 & 1 \\
1 & 2 & 1 \\
2 & 2 \\
1 & 1 & 0
\end{array}\right]\right]} \\
& {\left[[0,1],\left[\begin{array}{ccc}
0 & 1 \\
0 & 1 & 1 \\
0 & 1 \\
0 & 0 & 0
\end{array}\right],\left[\begin{array}{ccc}
1 & 0 \\
1 & 1 & 0 \\
1 & 0 \\
0 & 0 & 0
\end{array}\right]\right]}
\end{aligned}
$$

20.8.2. The sets $R_{[m, n]}(3),[m, n] \in L_{3}$

$$
\begin{aligned}
& {\left[[1,0],\left[\begin{array}{ccc}
0 & 0 \\
0 & 1 & 0 \\
1 & 1 \\
1 & 0 & 1
\end{array}\right],\left[\begin{array}{ccc}
0 & 0 \\
0 & 1 & 0 \\
1 & 1 \\
0 & 1 & 0
\end{array}\right],\left[\begin{array}{ccc}
0 & 0 \\
1 & 0 & 1 \\
1 & 1 \\
0 & 1 & 0
\end{array}\right]\right]} \\
& {\left[[2,0],\left[\begin{array}{ccc}
0 & 0 \\
1 & 1 & 1 \\
2 & 2 \\
0 & 2 & 0
\end{array}\right],\left[\begin{array}{ccc}
0 & 0 \\
1 & 1 & 1 \\
2 & 2 \\
1 & 1 & 1
\end{array}\right],\left[\begin{array}{ccc}
0 & 0 \\
0 & 2 & 0 \\
2 & 2 \\
1 & 1 & 1
\end{array}\right]\right]} \\
& {\left[[2,1],\left[\begin{array}{ccc}
0 & 0 \\
1 & 2 & 1 \\
2 & 2 \\
1 & 1 & 1
\end{array}\right],\left[\begin{array}{ccc}
1 & 1 \\
1 & 3 & 1 \\
3 & 3 \\
1 & 2 & 1
\end{array}\right],\left[\begin{array}{ccc}
0 & 0 \\
1 & 1 & 1 \\
2 & 2 & 2 \\
0 & 1 & 0
\end{array}\right]\right]} \\
& {\left[[1,1],\left[\begin{array}{ccc}
0 & 0 \\
0 & 1 & 0 \\
1 & 1 \\
0 & 0 & 0
\end{array}\right],\left[\begin{array}{ccc}
0 & 0 \\
1 & 1 & 1 \\
1 & 1 \\
0 & 1 & 0
\end{array}\right],\left[\begin{array}{ccc}
1 & 1 \\
1 & 2 & 1 \\
2 & 2 \\
1 & 1 & 1
\end{array}\right]\right]} \\
& {\left[[2,2],\left[\begin{array}{ccc}
1 & 1 \\
2 & 3 & 2 \\
3 & 3 \\
1 & 2 & 1
\end{array}\right],\left[\begin{array}{ccc}
1 & 1 \\
1 & 3 & 1 \\
3 & 3 \\
1 & 1 & 1
\end{array}\right],\left[\begin{array}{ccc}
0 & 0 \\
1 & 2 & 1 \\
2 & 2 \\
0 & 1 & 0
\end{array}\right]\right]} \\
& {\left[[1,2],\left[\begin{array}{ccc}
1 & 1 \\
1 & 2 & 1 \\
2 & 2 \\
0 & 1 & 0
\end{array}\right],\left[\begin{array}{ccc}
0 & 0 \\
1 & 1 & 1 \\
1 & 1 \\
0 & 0 & 0
\end{array}\right],\left[\begin{array}{ccc}
1 & 1 \\
1 & 3 & 1 \\
2 & 2 \\
1 & 1 & 1
\end{array}\right]\right]} \\
& {\left[[0,1],\left[\begin{array}{ccc}
0 & 0 \\
0 & 1 & 0 \\
0 & 0 \\
0 & 0 & 0
\end{array}\right],\left[\begin{array}{ccc}
1 & 1 \\
1 & 1 & 1 \\
1 & 1 \\
0 & 1 & 0
\end{array}\right],\left[\begin{array}{ccc}
0 & 0 \\
0 & 0 & 0 \\
0 & 0 \\
0 & -1 & 0
\end{array}\right]\right]}
\end{aligned}
$$

ANNALES SCIENTIFIQUES DE L'ÉCOLE NORMALE SUPÉRIEURE 


$$
\left[[0,2],\left[\begin{array}{ccc}
1 & 1 \\
1 & 1 & 1 \\
1 & 1 \\
0 & 0 & 0
\end{array}\right],\left[\begin{array}{ccc}
0 & 0 \\
0 & 1 & 0 \\
0 & 0 \\
0 & -1 & 0
\end{array}\right],\left[\begin{array}{ccc}
1 & 1 \\
1 & 2 & 1 \\
1 & 1 \\
0 & 1 & 0
\end{array}\right]\right]
$$

20.8.3. The sets $R_{[m, n]}(6),[m, n] \in L_{6}$

$\left[[1,0],\left[\begin{array}{ccc}-1 & 0 \\ 0 & 0 & 0 \\ 0 & 0 \\ 0 & 0 & 0\end{array}\right],\left[\begin{array}{ccc}1 & 0 \\ 1 & 1 & 0 \\ 1 & 1 \\ 0 & 1 & 1\end{array}\right],\left[\begin{array}{ccc}0 & 0 \\ 0 & 0 & 0 \\ 0 & 1 \\ 0 & 0 & 0\end{array}\right],\left[\begin{array}{ccc}0 & -1 \\ 0 & 0 & 0 \\ 0 & 0 \\ 0 & 0 & 0\end{array}\right],\left[\begin{array}{ccc}0 & 1 \\ 0 & 1 & 1 \\ 1 & 1 \\ 1 & 1 & 0\end{array}\right],\left[\begin{array}{ccc}0 & 0 \\ 0 & 0 & 0 \\ 1 & 0 \\ 0 & 0 & 0\end{array}\right]\right]$

$\left[[2,0],\left[\begin{array}{ccc}-1 & 0 \\ 0 & 0 & 0 \\ & 1 & 0 \\ 0 & 0 & 0\end{array}\right],\left[\begin{array}{ccc}0 & 0 \\ 1 & 1 & 0 \\ 1 & 1 \\ 0 & 1 & 1\end{array}\right],\left[\begin{array}{ccc}1 & 0 \\ 1 & 1 & 0 \\ 1 & 2 \\ 0 & 1 & 1\end{array}\right],\left[\begin{array}{ccc}0 & -1 \\ 0 & 0 & 0 \\ 0 & 1 \\ 0 & 0 & 0\end{array}\right],\left[\begin{array}{ccc}0 & 0 \\ 0 & 1 & 1 \\ 1 & 1 \\ 1 & 1 & 0\end{array}\right],\left[\begin{array}{ccc}0 & 1 \\ 0 & 1 & 1 \\ 2 & 1 \\ 1 & 1 & 0\end{array}\right]\right]$

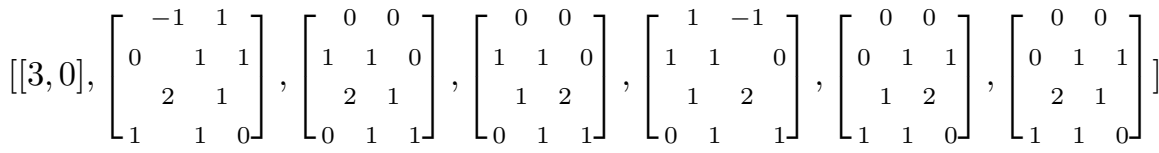

$\left[[4,0],\left[\begin{array}{ccc}1 & 0 \\ 1 & 2 & 1 \\ 2 & 3 \\ 1 & 2 & 1\end{array}\right],\left[\begin{array}{ccc}0 & 0 \\ 0 & 1 & 1 \\ 2 & 2 \\ 1 & 1 & 0\end{array}\right],\left[\begin{array}{ccc}-1 & 0 \\ 0 & 1 & 1 \\ 2 & 1 \\ 1 & 1 & 0\end{array}\right],\left[\begin{array}{ccc}0 & 1 \\ 1 & 2 & 1 \\ 3 & 2 \\ 1 & 2 & 1\end{array}\right],\left[\begin{array}{ccc}0 & 0 \\ 1 & 1 & 0 \\ 2 & 2 \\ 0 & 1 & 1\end{array}\right],\left[\begin{array}{ccc}0 & -1 \\ 1 & 1 & 0 \\ 1 & 2\end{array}\right]\right]$

$\left[[5,0],\left[\begin{array}{ccc}1 & 0 \\ 1 & 2 & 1 \\ 3 & 3 \\ 1 & 2 & 1\end{array}\right],\left[\begin{array}{ccc}-1 & 0 \\ 0 & \multicolumn{1}{c}{1} & 1 \\ 2 & 2 \\ 1 & 1 & 0\end{array}\right],\left[\begin{array}{ccc}0 & 0 \\ 1 & 2 & 1 \\ 3 & 2 \\ 1 & 2 & 1\end{array}\right],\left[\begin{array}{ccc}0 & 1 \\ 1 & 2 & 1 \\ 3 & 3 \\ 1 & 2 & 1\end{array}\right],\left[\begin{array}{cccc}0 & -1 \\ 1 & 1 & 0 \\ 2 & 2 & \\ 0 & 1 & 1\end{array}\right],\left[\begin{array}{ccc}0 & 0 \\ 1 & 2 & 1 \\ 2 & 3 \\ 1 & 2 & 1\end{array}\right]\right]$

$\left[[5,1],\left[\begin{array}{ccc}0 & 0 \\ 1 & 2 & 1 \\ 2 & 3 \\ 0 & 2 & 1\end{array}\right],\left[\begin{array}{ccc}1 & 0 \\ 2 & 2 & 1 \\ 3 & 3 \\ 1 & 2 & 1\end{array}\right],\left[\begin{array}{ccc}0 & 0 \\ 0 & 2 & 1 \\ 2 & 3 \\ 1 & 1 & 1\end{array}\right],\left[\begin{array}{ccc}0 & 0 \\ 1 & 2 & 1 \\ 3 & 2 \\ 1 & 2 & 0\end{array}\right],\left[\begin{array}{ccc}0 & 1 \\ 1 & 2 & 2 \\ 3 & 3 \\ 1 & 2 & 1\end{array}\right],\left[\begin{array}{ccc}0 & 0 \\ 1 & 2 & 0 \\ 3 & 2 \\ 1 & 1 & 1\end{array}\right]\right]$

$\left[[4,1],\left[\begin{array}{ccc}0 & 0 \\ 1 & 1 & 1 \\ 2 & 2 & 2 \\ 0 & 1 & 1\end{array}\right],\left[\begin{array}{ccc}0 & 0 \\ 1 & 2 & 0 \\ 2 & 2 \\ 1 & 1 & 1\end{array}\right],\left[\begin{array}{ccc}1 & 0 \\ 1 & 2 & 1 \\ 2 & 3 \\ 0 & 2 & 1\end{array}\right],\left[\begin{array}{ccc}0 & 0 \\ 1 & 1 & 1 \\ 2 & 2 \\ 1 & 1 & 0\end{array}\right],\left[\begin{array}{ccc}0 & 0 \\ 0 & 2 & 1 \\ 2 & 2 \\ 1 & 1 & 1\end{array}\right],\left[\begin{array}{ccc}0 & 1 \\ 1 & 2 & 1 \\ 3 & 2 \\ 1 & 2 & 0\end{array}\right]\right]$

$\left[[3,1],\left[\begin{array}{ccc}0 & 0 \\ 1 & 1 & 0 \\ 2 & 1 \\ 0 & 1 & 0\end{array}\right],\left[\begin{array}{ccc}0 & 0 \\ 1 & 1 & 1 \\ 1 & 2 \\ 0 & 1 & 1\end{array}\right],\left[\begin{array}{ccc}1 & 0 \\ 1 & 2 & 0 \\ 2 & 2 \\ 1 & 1 & 1\end{array}\right],\left[\begin{array}{ccc}0 & 0 \\ 0 & 1 & 1 \\ 1 & 2 \\ 0 & 1 & 0\end{array}\right],\left[\begin{array}{ccc}0 & 0 \\ 1 & 1 & 1 \\ 2 & 1 \\ 1 & 1 & 0\end{array}\right],\left[\begin{array}{ccc}0 & 1 \\ 0 & 2 & 1 \\ 2 & 2 \\ 1 & 1 & 1\end{array}\right]\right]$

$\left[[5,2],\left[\begin{array}{ccc}0 & 0 \\ 1 & 2 & 1 \\ 2 & 3 \\ 1 & 1 & 1\end{array}\right],\left[\begin{array}{ccc}1 & 0 \\ 1 & 3 & 1 \\ 3 & 3 \\ 1 & 2 & 1\end{array}\right],\left[\begin{array}{ccc}0 & 1 \\ 1 & 2 & 2 \\ 3 & 3 \\ 1 & 2 & 0\end{array}\right],\left[\begin{array}{ccc}0 & 0 \\ 1 & 2 & 1 \\ 3 & 2 \\ 1 & 1 & 1\end{array}\right],\left[\begin{array}{ccc}0 & 1 \\ 1 & 3 & 1 \\ 3 & 3 \\ 1 & 2 & 1\end{array}\right],\left[\begin{array}{ccc}1 & 0 \\ 2 & 2 & 1 \\ 3 & 3 \\ 0 & 2 & 1\end{array}\right]\right]$

$\left[[2,1],\left[\begin{array}{ccc}0 & 0 \\ 0 & 1 & 0 \\ 1 & 1 \\ 0 & 0 & 1\end{array}\right],\left[\begin{array}{ccc}0 & 0 \\ 1 & 1 & 0 \\ 1 & 1 \\ 0 & 1 & 0\end{array}\right],\left[\begin{array}{ccc}1 & 0 \\ 1 & 1 & 1 \\ 1 & 2 \\ 0 & 1 & 1\end{array}\right],\left[\begin{array}{ccc}0 & 0 \\ 0 & 1 & 0 \\ 1 & 1 \\ 1 & 0 & 0\end{array}\right],\left[\begin{array}{ccc}0 & 0 \\ 0 & 1 & 1 \\ 1 & 1 \\ 0 & 1 & 0\end{array}\right],\left[\begin{array}{ccc}0 & 1 \\ 1 & 1 & 1 \\ 2 & 1 \\ 1 & 1 & 0\end{array}\right]\right]$ 
$\left[[4,2],\left[\begin{array}{ccc}0 & 0 \\ 0 & 2 & 1 \\ 2 & 2 \\ 1 & 1 & 0\end{array}\right],\left[\begin{array}{ccc}0 & 1 \\ 1 & 2 & 2 \\ 3 & 2 \\ 1 & 2 & 0\end{array}\right],\left[\begin{array}{ccc}0 & 1 \\ 1 & 2 & 1 \\ 3 & 2 \\ 1 & 1 & 1\end{array}\right],\left[\begin{array}{ccc}0 & 0 \\ 1 & 2 & 0 \\ 2 & 2 \\ 0 & 1 & 1\end{array}\right],\left[\begin{array}{ccc}1 & 0 \\ 2 & 2 & 1 \\ 2 & 3 \\ 0 & 2 & 1\end{array}\right],\left[\begin{array}{ccc}1 & 0 \\ 1 & 2 & 1 \\ 2 & 3 \\ 1 & 1 & 1\end{array}\right]\right]$

$\left[[5,3],\left[\begin{array}{ccc}1 & 0 \\ 2 & 3 & 1 \\ 3 & 3 \\ 1 & 2 & 1\end{array}\right],\left[\begin{array}{ccc}1 & 1 \\ 1 & 3 & 2 \\ 3 & 4 \\ 1 & 2 & 1\end{array}\right],\left[\begin{array}{ccc}0 & 0 \\ 1 & 2 & 1 \\ 3 & 2 \\ 1 & 1 & 0\end{array}\right],\left[\begin{array}{ccc}0 & 1 \\ 1 & 3 & 2 \\ 3 & 3 \\ 1 & 2 & 1\end{array}\right],\left[\begin{array}{ccc}1 & 1 \\ 2 & 3 & 1 \\ 4 & 3 \\ 1 & 2 & 1\end{array}\right],\left[\begin{array}{ccc}0 & 0 \\ 1 & 2 & 1 \\ 2 & 3 \\ 0 & 1 & 1\end{array}\right]\right]$

$\left[[3,2],\left[\begin{array}{ccc}0 & 0 \\ 1 & 1 & 1 \\ 2 & 1 \\ 0 & 1 & 0\end{array}\right],\left[\begin{array}{ccc}0 & 1 \\ 1 & 2 & 1 \\ 2 & 2 \\ 1 & 1 & 1\end{array}\right],\left[\begin{array}{ccc}1 & 0 \\ 1 & 2 & 0 \\ 2 & 2 \\ 0 & 1 & 1\end{array}\right],\left[\begin{array}{ccc}0 & 0 \\ 1 & 1 & 1 \\ 1 & 2 \\ 0 & 1 & 0\end{array}\right],\left[\begin{array}{ccc}1 & 0 \\ 1 & 2 & 1 \\ 2 & 2 \\ 1 & 1 & 1\end{array}\right],\left[\begin{array}{ccc}0 & 1 \\ 0 & 2 & 1 \\ 2 & 2 \\ 1 & 1 & 0\end{array}\right]\right]$

$\left[[4,3],\left[\begin{array}{ccc}0 & 0 \\ 1 & 2 & 1 \\ 2 & 2 & 2 \\ 1 & 1 & 0\end{array}\right],\left[\begin{array}{ccc}1 & 1 \\ 1 & 3 & 2 \\ 3 & 3 \\ 1 & 2 & 1\end{array}\right],\left[\begin{array}{ccc}0 & 1 \\ 1 & 2 & 1 \\ 3 & 2 \\ 1 & 1 & 0\end{array}\right],\left[\begin{array}{ccc}0 & 0 \\ 1 & 2 & 1 \\ 2 & 2 \\ 0 & 1 & 1\end{array}\right],\left[\begin{array}{ccc}1 & 1 \\ 2 & 3 & 1 \\ 3 & 3 \\ 1 & 2 & 1\end{array}\right],\left[\begin{array}{ccc}1 & 0 \\ 1 & 2 & 1 \\ 2 & 3 \\ 0 & 1 & 1\end{array}\right]\right]$

$\left[[5,4],\left[\begin{array}{ccc}0 & 1 \\ 1 & 3 & 2 \\ 3 & 3 \\ 1 & 2 & 0\end{array}\right],\left[\begin{array}{ccc}1 & 1 \\ 2 & 3 & 2 \\ 4 & 3 \\ 1 & 2 & 1\end{array}\right],\left[\begin{array}{ccc}0 & 1 \\ 1 & 3 & 1 \\ 3 & 3 \\ 1 & 1 & 1\end{array}\right],\left[\begin{array}{ccc}1 & 0 \\ 2 & 3 & 1 \\ 3 & 3 \\ 0 & 2 & 1\end{array}\right],\left[\begin{array}{ccc}1 & 1 \\ 2 & 3 & 2 \\ 3 & 4 \\ 1 & 2 & 1\end{array}\right],\left[\begin{array}{ccc}1 & 0 \\ 1 & 3 & 1 \\ 3 & 3 \\ 1 & 1 & 1\end{array}\right]\right]$

$\left[[1,1],\left[\begin{array}{ccc}0 & 0 \\ 1 & 0 & 0 \\ 1 & 0 \\ 0 & 0 & 0\end{array}\right],\left[\begin{array}{ccc}0 & 0 \\ 0 & 1 & 0 \\ 0 & 1 \\ 0 & 0 & 1\end{array}\right],\left[\begin{array}{ccc}1 & 0 \\ 1 & 1 & 0 \\ 1 & 1 \\ 0 & 1 & 0\end{array}\right],\left[\begin{array}{ccc}0 & 0 \\ 0 & 0 & 1 \\ 0 & 1 \\ 0 & 0 & 0\end{array}\right],\left[\begin{array}{ccc}0 & 0 \\ 0 & 1 & 0 \\ 1 & 0 \\ 1 & 0 & 0\end{array}\right],\left[\begin{array}{ccc}0 & 1 \\ 0 & 1 & 1 \\ 1 & 1 \\ 0 & 1 & 0\end{array}\right]\right]$

$\left[[2,2],\left[\begin{array}{ccc}0 & 1 \\ 1 & 1 & 1 \\ 2 & 1 \\ 0 & 1 & 0\end{array}\right],\left[\begin{array}{ccc}0 & 0 \\ 1 & 1 & 0 \\ 1 & 1 \\ 0 & 0 & 1\end{array}\right],\left[\begin{array}{ccc}1 & 0 \\ 1 & 2 & 0 \\ 1 & 2 \\ 0 & 1 & 1\end{array}\right],\left[\begin{array}{ccc}1 & 0 \\ 1 & 1 & 1 \\ 1 & 2 \\ 0 & 1 & 0\end{array}\right],\left[\begin{array}{ccc}0 & 0 \\ 0 & 1 & 1 \\ 1 & 1 \\ 1 & 0 & 0\end{array}\right],\left[\begin{array}{ccc}0 & 1 \\ 0 & 2 & 1 \\ 2 & 1 \\ 1 & 1 & 0\end{array}\right]\right]$

$\left[[3,3],\left[\begin{array}{ccc}0 & 1 \\ 1 & 2 & 1 \\ 3 & 1 \\ 1 & 1 & 0\end{array}\right],\left[\begin{array}{ccc}0 & 1 \\ 1 & 2 & 1 \\ 2 & 2 \\ 0 & 1 & 1\end{array}\right],\left[\begin{array}{ccc}1 & 0 \\ 2 & 2 & 0 \\ 2 & 2 \\ 0 & 1 & 1\end{array}\right],\left[\begin{array}{ccc}1 & 0 \\ 1 & 2 & 1 \\ 1 & 3 \\ 0 & 1 & 1\end{array}\right],\left[\begin{array}{ccc}1 & 0 \\ 1 & 2 & 1 \\ 2 & 2 \\ 1 & 1 & 0\end{array}\right],\left[\begin{array}{ccc}0 & 1 \\ 0 & 2 & 2 \\ 2 & 2 \\ 1 & 1 & 0\end{array}\right]\right]$

$\left[[4,4],\left[\begin{array}{ccc}1 & 0 \\ 1 & 3 & 1 \\ 2 & 3 & 3\end{array}\right],\left[\begin{array}{ccc}1 & 1 \\ 1 & 3 & 2 \\ 3 & 3 & 3 \\ 1 & 2 & 0\end{array}\right],\left[\begin{array}{ccc}0 & 1 \\ 1 & 2 & 2 \\ 3 & 2 \\ 1 & 1 & 0\end{array}\right],\left[\begin{array}{ccc}0 & 1 \\ 1 & 3 & 1 \\ 3 & 2 \\ 1 & 1 & 1\end{array}\right],\left[\begin{array}{ccc}1 & 1 \\ 2 & 3 & 1 \\ 3 & 3 \\ 0 & 2 & 1\end{array}\right],\left[\begin{array}{ccc}1 & 0 \\ 2 & 2 & 1 \\ 2 & 3 \\ 0 & 1 & 1\end{array}\right]\right]$

$\left[[5,5],\left[\begin{array}{ccc}1 & 1 \\ 1 & 4 & 2 \\ 3 & 4 \\ 1 & 2 & 1\end{array}\right],\left[\begin{array}{ccc}1 & 1 \\ 2 & 3 & 2 \\ 4 & 3 \\ 1 & 2 & 0\end{array}\right],\left[\begin{array}{ccc}0 & 1 \\ 1 & 3 & 2 \\ 3 & 3 \\ 1 & 1 & 1\end{array}\right],\left[\begin{array}{ccc}1 & 1 \\ 2 & 4 & 1 \\ 4 & 3 \\ 1 & 2 & 1\end{array}\right],\left[\begin{array}{ccc}1 & 1 \\ 2 & 3 & 2 \\ 3 & 4 \\ 0 & 2 & 1\end{array}\right],\left[\begin{array}{ccc}1 & 0 \\ 2 & 3 & 1 \\ 3 & 3 \\ 1 & 1 & 1\end{array}\right]\right]$

$\left[[4,5],\left[\begin{array}{ccc}1 & 1 \\ 2 & 3 & 2 \\ 3 & 3 \\ 1 & 2 & 0\end{array}\right],\left[\begin{array}{ccc}1 & 1 \\ 1 & 3 & 2 \\ 3 & 3 \\ 1 & 1 & 1\end{array}\right],\left[\begin{array}{ccc}0 & 1 \\ 1 & 3 & 1 \\ 3 & 2 \\ 1 & 1 & 0\end{array}\right],\left[\begin{array}{ccc}1 & 1 \\ 2 & 3 & 2 \\ 3 & 3 \\ 0 & 2 & 1\end{array}\right],\left[\begin{array}{ccc}1 & 1 \\ 2 & 3 & 1 \\ 3 & 3 \\ 1 & 1 & 1\end{array}\right],\left[\begin{array}{ccc}1 & 0 \\ 1 & 3 & 1 \\ 2 & 3 \\ 0 & 1 & 1\end{array}\right]\right]$

$\left[[3,4],\left[\begin{array}{ccc}1 & 1 \\ 1 & 3 & 1 \\ 2 & 3 & 3 \\ 1 & 1 & 1\end{array}\right],\left[\begin{array}{ccc}1 & 0 \\ 1 & 2 & 1 \\ 2 & 2 \\ 0 & 1 & 0\end{array}\right],\left[\begin{array}{ccc}0 & 1 \\ 1 & 2 & 2 \\ 2 & 2 \\ 1 & 1 & 0\end{array}\right],\left[\begin{array}{ccc}1 & 1 \\ 1 & 3 & 1 \\ 3 & 2 \\ 1 & 1 & 1\end{array}\right],\left[\begin{array}{ccc}0 & 1 \\ 1 & 2 & 1 \\ 2 & 2 \\ 0 & 1 & 0\end{array}\right],\left[\begin{array}{ccc}1 & 0 \\ 2 & 2 & 1 \\ 2 & 2 \\ 0 & 1 & 1\end{array}\right]\right]$ 


$$
\begin{aligned}
& {\left[[2,3],\left[\begin{array}{ccc}
1 & 1 \\
1 & 2 & 1 \\
2 & 2 \\
0 & 1 & 1
\end{array}\right],\left[\begin{array}{ccc}
0 & 0 \\
1 & 1 & 0 \\
1 & 1 \\
0 & 0 & 0
\end{array}\right],\left[\begin{array}{ccc}
1 & 0 \\
1 & 2 & 1 \\
1 & 2 \\
0 & 1 & 1
\end{array}\right],\left[\begin{array}{ccc}
1 & 1 \\
1 & 2 & 1 \\
2 & 2 \\
1 & 1 & 0
\end{array}\right],\left[\begin{array}{ccc}
0 & 0 \\
0 & 1 & 1 \\
1 & 1 \\
0 & 0 & 0
\end{array}\right],\left[\begin{array}{ccc}
0 & 1 \\
1 & 2 & 1 \\
2 & 1 \\
1 & 1 & 0
\end{array}\right]\right]} \\
& {\left[[3,5],\left[\begin{array}{ccc}
1 & 1 \\
1 & 3 & 2 \\
2 & 3 \\
1 & 1 & 1
\end{array}\right],\left[\begin{array}{ccc}
1 & 1 \\
1 & 3 & 1 \\
3 & 2 \\
1 & 1 & 0
\end{array}\right],\left[\begin{array}{ccc}
0 & 1 \\
1 & 2 & 2 \\
2 & 2 \\
0 & 1 & 0
\end{array}\right],\left[\begin{array}{ccc}
1 & 1 \\
2 & 3 & 1 \\
3 & 2 \\
1 & 1 & 1
\end{array}\right],\left[\begin{array}{ccc}
1 & 1 \\
1 & 3 & 1 \\
2 & 3 \\
0 & 1 & 1
\end{array}\right],\left[\begin{array}{ccc}
1 & 0 \\
2 & 2 & 1 \\
2 & 2 \\
0 & 1 & 0
\end{array}\right]\right]} \\
& {\left[[1,2],\left[\begin{array}{ccc}
1 & 0 \\
1 & 1 & 0 \\
1 & 1 \\
0 & 0 & 1
\end{array}\right],\left[\begin{array}{ccc}
0 & 0 \\
0 & 1 & 0 \\
0 & 1 \\
0 & 0 & 0
\end{array}\right],\left[\begin{array}{ccc}
1 & 0 \\
1 & 1 & 1 \\
1 & 1 \\
0 & 1 & 0
\end{array}\right],\left[\begin{array}{ccc}
0 & 1 \\
0 & 1 & 1 \\
1 & 1 \\
1 & 0 & 0
\end{array}\right],\left[\begin{array}{ccc}
0 & 0 \\
0 & 1 & 0 \\
1 & 0 \\
0 & 0 & 0
\end{array}\right],\left[\begin{array}{ccc}
0 & 1 \\
1 & 1 & 1 \\
1 & 1 \\
0 & 1 & 0
\end{array}\right]\right]} \\
& {\left[[2,4],\left[\begin{array}{ccc}
1 & 1 \\
2 & 2 & 1 \\
2 & 2 \\
0 & 1 & 1
\end{array}\right],\left[\begin{array}{ccc}
1 & 0 \\
1 & 2 & 0 \\
1 & 2 \\
0 & 0 & 1
\end{array}\right],\left[\begin{array}{ccc}
1 & 0 \\
1 & 2 & 1 \\
1 & 2 \\
0 & 1 & 0
\end{array}\right],\left[\begin{array}{ccc}
1 & 1 \\
1 & 2 & 2 \\
2 & 2 \\
1 & 1 & 0
\end{array}\right],\left[\begin{array}{ccc}
0 & 1 \\
0 & 2 & 1 \\
2 & 1 \\
1 & 0 & 0
\end{array}\right],\left[\begin{array}{ccc}
0 & 1 \\
1 & 2 & 1 \\
2 & 1 \\
0 & 1 & 0
\end{array}\right]\right]} \\
& {\left[[2,5],\left[\begin{array}{ccc}
1 & 1 \\
1 & 3 & 1 \\
2 & 2 & 2 \\
1 & 1 & 0
\end{array}\right],\left[\begin{array}{ccc}
1 & 1 \\
1 & 2 & 2 \\
2 & 2 \\
0 & 1 & 0
\end{array}\right],\left[\begin{array}{ccc}
0 & 1 \\
1 & 2 & 1 \\
2 & 1 \\
1 & 0 & 0
\end{array}\right],\left[\begin{array}{ccc}
1 & 1 \\
1 & 3 & 1 \\
2 & 2
\end{array}\right],\left[\begin{array}{ccc}
1 & 1 \\
2 & 2 & 1 \\
2 & 1 & 2
\end{array}\right],\left[\begin{array}{ccc}
1 & 0 \\
1 & 2 & 1 \\
1 & 2 \\
0 & 0 & 1
\end{array}\right]\right]} \\
& {\left[[1,3],\left[\begin{array}{ccc}
0 & 1 \\
0 & 1 & 1 \\
1 & 1 \\
0 & 0 & 0
\end{array}\right],\left[\begin{array}{ccc}
0 & 0 \\
1 & 1 & 0 \\
1 & 0 \\
0 & 0 & 0
\end{array}\right],\left[\begin{array}{ccc}
1 & 1 \\
1 & 2 & 1 \\
1 & 2 \\
0 & 1 & 1
\end{array}\right],\left[\begin{array}{ccc}
1 & 0 \\
1 & 1 & 0 \\
1 & 1 \\
0 & 0 & 0
\end{array}\right],\left[\begin{array}{ccc}
0 & 0 \\
0 & 1 & 1 \\
0 & 1 \\
0 & 0 & 0
\end{array}\right],\left[\begin{array}{ccc}
1 & 1 \\
1 & 2 & 1 \\
2 & 1 \\
1 & 1 & 0
\end{array}\right]\right]} \\
& {\left[[1,4],\left[\begin{array}{ccc}
1 & 1 \\
1 & 2 & 1 \\
2 & 1 \\
0 & 1 & 0
\end{array}\right],\left[\begin{array}{ccc}
0 & 1 \\
1 & 1 & 1 \\
1 & 1 \\
0 & 0 & 0
\end{array}\right],\left[\begin{array}{ccc}
1 & 0 \\
1 & 2 & 0 \\
1 & 1 \\
0 & 0 & 1
\end{array}\right],\left[\begin{array}{ccc}
1 & 1 \\
1 & 2 & 1 \\
1 & 2
\end{array}\right],\left[\begin{array}{ccc}
1 & 0 \\
1 & 1 & 1 \\
1 & 1 & 1
\end{array}\right],\left[\begin{array}{ccc}
0 & 1 \\
0 & 2 & 1 \\
1 & 0 & 1 \\
1 & 0 & 0
\end{array}\right]\right]} \\
& {\left[[1,5],\left[\begin{array}{ccc}
1 & 1 \\
1 & 2 & 1 \\
2 & 1 \\
1 & 0 & 0
\end{array}\right],\left[\begin{array}{ccc}
0 & 1 \\
0 & 2 & 1 \\
1 & 1 \\
0 & 0 & 0
\end{array}\right],\left[\begin{array}{ccc}
1 & 1 \\
2 & 2 & 1 \\
2 & 1 \\
0 & 1 & 0
\end{array}\right],\left[\begin{array}{ccc}
1 & 1 \\
1 & 2 & 1 \\
1 & 2 \\
0 & 0 & 1
\end{array}\right],\left[\begin{array}{ccc}
1 & 0 \\
1 & 2 & 0 \\
1 & 1 \\
0 & 0 & 0
\end{array}\right],\left[\begin{array}{ccc}
1 & 1 \\
1 & 2 & 2 \\
1 & 2 \\
0 & 1 & 0
\end{array}\right]\right]} \\
& {\left[[0,1],\left[\begin{array}{ccc}
0 & 0 \\
1 & 0 & 0 \\
0 & 0 \\
0 & 0 & 0
\end{array}\right],\left[\begin{array}{ccc}
1 & 0 \\
0 & 1 & 0 \\
0 & 1 & 1 \\
0 & 0 & 1
\end{array}\right],\left[\begin{array}{ccc}
0 & 0 \\
0 & 0 & 0 \\
0 & 0 \\
0 & 0 & -1
\end{array}\right],\left[\begin{array}{ccc}
0 & 0 \\
0 & 0 & 1 \\
0 & 0 \\
0 & 0 & 0
\end{array}\right],\left[\begin{array}{ccc}
0 & 1 \\
0 & 1 & 0 \\
1 & 0 \\
1 & 0 & 0
\end{array}\right],\left[\begin{array}{ccc}
0 & 0 \\
0 & 0 & 0 \\
0 & 0 \\
-1 & 0 & 0
\end{array}\right]\right]} \\
& {\left[[0,2],\left[\begin{array}{ccc}
0 & 0 \\
1 & 0 & 0 \\
0 & 0 & 0 \\
-1 & 0 & 0
\end{array}\right],\left[\begin{array}{ccc}
1 & 0 \\
1 & 1 & 0 \\
0 & 1 \\
0 & 0 & 1
\end{array}\right],\left[\begin{array}{ccc}
1 & 0 \\
0 & 1 & 0 \\
0 & 1 \\
0 & 0 & 0
\end{array}\right],\left[\begin{array}{ccc}
0 & 0 \\
0 & 0 & 1 \\
0 & 0 & 1 \\
0 & 0 & -1
\end{array}\right],\left[\begin{array}{ccc}
0 & 1 \\
0 & 1 & 1 \\
1 & 0 \\
1 & 0 & 0
\end{array}\right],\left[\begin{array}{ccc}
0 & 1 \\
0 & 1 & 0 \\
1 & 0 \\
0 & 0 & 0
\end{array}\right]\right]} \\
& {\left[[0,3],\left[\begin{array}{ccc}
0 & 1 \\
1 & 1 & 0 \\
1 & 0 \\
0 & 0 & 0
\end{array}\right],\left[\begin{array}{ccc}
1 & 0 \\
1 & 1 & 0 \\
0 & 0 & 1 \\
-1 & 0 & 1
\end{array}\right],\left[\begin{array}{ccc}
1 & 0 \\
1 & 1 & 0 \\
0 & 1 \\
0 & 0 & 0
\end{array}\right],\left[\begin{array}{ccc}
1 & 0 \\
0 & 1 & 1 \\
0 & 1 \\
0 & 0 & 0
\end{array}\right],\left[\begin{array}{ccc}
0 & 1 \\
0 & 1 & 1 \\
1 & 0 \\
1 & 0 & -1
\end{array}\right],\left[\begin{array}{ccc}
0 & 1 \\
0 & 1 & 1 \\
1 & 0 \\
0 & 0 & 0
\end{array}\right]\right]} \\
& {\left[[0,4],\left[\begin{array}{ccc}
1 & 1 \\
0 & 2 & 1 \\
1 & 1 \\
1 & 0 & 0
\end{array}\right],\left[\begin{array}{ccc}
0 & 1 & 1 \\
0 & 1 & 1 \\
1 & 0 & \\
0 & 0 & -1
\end{array}\right],\left[\begin{array}{ccc}
0 & 1 \\
1 & 1 & 1 \\
1 & 0 \\
0 & 0 & 0
\end{array}\right],\left[\begin{array}{ccc}
1 & 1 \\
1 & 2 & 0 \\
1 & 1 \\
0 & 0 & 1
\end{array}\right],\left[\begin{array}{ccc}
1 & 0 \\
1 & 1 & 0 \\
0 & 1 \\
-1 & 0 & 0
\end{array}\right],\left[\begin{array}{ccc}
1 & 0 \\
1 & 1 & 1 \\
0 & 1 \\
0 & 0 & 0
\end{array}\right]\right]}
\end{aligned}
$$




$$
\left[[0,5],\left[\begin{array}{ccc}
1 & 1 \\
0 & 2 & 1 \\
1 & 1 \\
0 & 0 & 0
\end{array}\right],\left[\begin{array}{ccc}
0 & 1 & \\
1 & 1 & 1 \\
1 & 0 \\
0 & 0 & -1
\end{array}\right],\left[\begin{array}{ccc}
1 & 1 \\
1 & 2 & 1 \\
1 & 1 \\
0 & 0 & 1
\end{array}\right],\left[\begin{array}{ccc}
1 & 1 \\
1 & 2 & 0 \\
1 & 1 \\
0 & 0 & 0
\end{array}\right],\left[\begin{array}{ccc}
1 & 0 \\
1 & 1 & 1 \\
0 & 1 \\
-1 & 0 & 0
\end{array}\right],\left[\begin{array}{ccc}
1 & 1 \\
1 & 2 & 1 \\
1 & 1 \\
1 & 0 & 0
\end{array}\right]\right]
$$

\section{Acknowledgements}

C. Geiss acknowledges support from DGAPA-UNAM for a sabbatical stay at the University of Leeds. J. Schröer was supported by a research fellowship from the DFG (Deutsche Forschungsgemeinschaft). He also thanks the Laboratoire LMNO (Caen) for an invitation in Spring 2003 during which this work was started. B. Leclerc is grateful to the GDR 2432 (Algèbre non commutative) and the GDR 2249 (Groupes, géométrie et représentations) for their support.

\section{REFERENCES}

[1] Auslander M., Reiten I., Smalø S., Representation Theory of Artin Algebras, Cambridge Studies in Advanced Mathematics, vol. 36, Cambridge University Press, Cambridge, 1997, Corrected reprint of the 1995 original. xiv+425pp.

[2] Bautista R., On algebras of strongly unbounded representation type, Comment. Math. Helv. 60 (3) (1985) 392-399.

[3] Berenstein A., Fomin S., Zelevinsky A., Cluster algebras III: Upper bounds and double Bruhat cells, Duke Math. J. 126 (1) (2005) 1-52.

[4] Berenstein A., Zelevinsky A., String bases for quantum groups of type $A_{r}$, in: I.M. Gelfand Seminar, in: Adv. Soviet Math., vol. 16, Amer. Math. Soc., Providence, RI, 1993, pp. 51-89.

[5] Bobiński G., Skowroński A., Geometry of modules over tame quasi-tilted algebras, Colloq. Math. 79 (1) (1999) 85-118.

[6] Bongartz K., Algebras and quadratic forms, J. London Math. Soc. 28 (3) (1983) 461-469.

[7] Bongartz K., A geometric version of the Morita equivalence, J. Algebra 139 (1) (1991) 159-171.

[8] CALdero P., Adapted algebras for the Berenstein Zelevinsky conjecture, Transform. Groups 8 (1) (2003) 37-50.

[9] CALDERo P., A multiplicative property of quantum flag minors, Representation Theory 7 (2003) 164 176.

[10] Caldero P., MARSh R., A multiplicative property of quantum flag minors II, J. London Math. Soc. 69 (3) (2004) 608-622.

[11] Chapoton F., Fomin S., Zelevinsky A., Polytopal realizations of generalized associahedra, Canad. Math. Bull. 45 (2002) 537-566.

[12] Crawley-Boevey W., On the exceptional fibres of Kleinian singularities, Amer. J. Math. 122 (2000) 1027-1037.

[13] Crawley-Boevey W., Geometry of the moment map for representations of quivers, Compositio Math. 126 (2001) 257-293.

[14] Crawley-Boevey W., Schröer J., Irreducible components of varieties of modules, J. Reine Angew. Math. 553 (2002) 201-220.

[15] Dlab V., Ringel C.M., The module theoretical approach to quasi-hereditary algebras, in: Representations of Algebras and Related Topics, Kyoto, 1990, Cambridge Univ. Press, Cambridge, 1992, pp. 200-224.

[16] Dowbor P., Skowroński A., On Galois coverings of tame algebras, Arch. Math. 44 (1985) 522529.

[17] Dowbor P., Skowroński A., Galois coverings of representation-infinite algebras, Comment. Math. Helv. 62 (1987) 311-337.

[18] Fomin S., Zelevinsky A., Cluster algebras I: Foundations, J. Amer. Math. Soc. 15 (2002) 497-529.

[19] Fomin S., ZELEVINSKy A., Y-systems and generalized associahedra, Annals of Math. 158 (3) (2003) 977-1018. 
[20] Fomin S., Zelevinsky A., Cluster algebras II: Finite type classification, Invent. Math. 154 (2003) 63-121.

[21] GABRIEL P., Auslander-Reiten sequences and representation-finite algebras, in: Representation Theory I, Carleton, 1979, in: Lecture Notes in Math., vol. 831, Springer-Verlag, Berlin, 1980, pp. 171.

[22] GABRIEL P., The universal cover of a representation-finite algebra, in: Representations of Algebras, Puebla, 1980, in: Lecture Notes in Math., vol. 903, Springer-Verlag, Berlin, 1981, pp. 68-105.

[23] Geigle W., Lenzing H., A class of weighted projective curves arising in representation theory of finite-dimensional algebras, in: Singularities, Representation of Algebras, and Vector Bundles, Lambrecht, 1985, in: Lecture Notes in Math., vol. 1273, Springer-Verlag, Berlin, 1987, pp. 265297.

[24] GeISS C., SCHRÖER J., Varieties of modules over tubular algebras, Colloq. Math. 95 (2003) 163-183.

[25] GeISS C., SCHRÖER J., Extension-orthogonal components of nilpotent varieties, Trans. Amer. Math. Soc. 357 (2005) 1953-1962.

[26] HAPPEL D., Triangulated Categories in the Representation Theory of Finite-Dimensional Algebras, London Mathematical Society Lecture Note Series, vol. 119, Cambridge University Press, Cambridge, 1988, x+208pp.

[27] Happel D., Ringel C.M., The derived category of a tubular algebra, in: Representation Theory, I, Ottawa, Ont., 1984, in: Lecture Notes in Math., vol. 1177, Springer-Verlag, Berlin, 1986, pp. 156180.

[28] Happel D., Vossieck D., Minimal algebras of infinite representation type with preprojective component, Manuscripta Math. 42 (1983) 221-243.

[29] Kashiwara M., On crystal bases of the $q$-analogue of universal enveloping algebras, Duke Math. J. 63 (1991) 465-516.

[30] Kashiwara M., SAito Y., Geometric construction of crystal bases, Duke Math. J. 89 (1997) 9-36.

[31] LECLERC B., Imaginary vectors in the dual canonical basis of $U_{q}(n)$, Transform. Groups 8 (2003) 95-104.

[32] LeCLERC B., Dual canonical bases, quantum shuffles and q-characters, Math. Z. 246 (2004) 691-732.

[33] Leclerc B., NAZArov M., Thibon J.-Y., Induced representations of affine Hecke algebras and canonical bases of quantum groups, in: Studies in Memory of Issai Schur, in: Progress in Mathematics, vol. 210, Birkhäuser, Basel, 2003.

[34] LenZING H., A K-theoretic study of canonical algebras, in: CMS Conf. Proc., vol. 18, 1996, pp. 433 454.

[35] LENZING H., Coxeter transformations associated with finite dimensional algebras, in: Computational Methods for Representations of Groups and Algebras, Essen, 1997, in: Progress in Math., vol. 173, Birkhäuser, Basel, 1999.

[36] Lenzing H., Meltzer H., Sheaves on a weighted projective line of genus one, and representations of a tubular algebra, in: Representations of Algebras, Ottawa, ON, 1992, in: CMS Conf. Proc., vol. 14, 1993, pp. 313-337.

[37] Lusztig G., Canonical bases arising from quantized enveloping algebras, J. Amer. Math. Soc. 3 (1990) 447-498.

[38] Lusztig G., Quivers, perverse sheaves and quantized enveloping algebras, J. Amer. Math. Soc. 4 (1991) 365-421.

[39] Lusztig G., Affine quivers and canonical bases, Publ. Math. IHES 76 (1994) 365-416.

[40] Lusztig G., Constructible functions on the Steinberg variety, Adv. Math. 130 (1997) 365-421.

[41] Lusztig G., Semicanonical bases arising from enveloping algebras, Adv. Math. 151 (2000) 129-139.

[42] MARsh R., ReINeKe M., Personal communication.

[43] REINEKE M., Multiplicative properties of dual canonical bases of quantum groups, J. Algebra 211 (1999) 134-149.

[44] REITEN I., Dynkin diagrams and the representation theory of algebras, Notices AMS 44 (1997) 546556.

[45] Ringel C.M., Tame Algebras and Integral Quadratic Forms, Lecture Notes in Math., vol. 1099, Springer-Verlag, Berlin, 1984, xiii+376pp.

$4^{\text {e }}$ SÉRIE - TOME $38-2005-\mathrm{N}^{\circ} 2$ 
[46] Ringel C.M., The preprojective algebra of a quiver, in: Algebras and Modules II, Geiranger, 1966, in: CMS Conf. Proc., vol. 24, AMS, 1998, pp. 467-480.

[47] Ringel C.M., The multisegment duality and the preprojective algebras of type A, Algebra Montpellier Announcements 1.1 (1999) (6 pages).

[48] Ringel C.M., Representation theory of finite-dimensional algebras, in: Representations of Algebras, Proceedings of the Durham Symposium 1985, in: Lecture Note Series, vol. 116, LMS, 1986, pp. 9 79.

[49] SCHRÖER J., Module theoretic interpretation of quantum minors, in preparation.

[50] Saito K., Extended affine root systems I, Publ. Res. Inst. Math. Sci. 21 (1985) 75-179.

[51] Zelevinsky A., Personal communication.

[52] Zelevinsky A., The multisegment duality, in: Documenta Mathematica, Extra Volume, ICM, Berlin, 1998, III, pp. 409-417.

(Manuscrit reçu le 3 juin 2004;

accepté le 16 décembre 2004.)

\footnotetext{
Christof GeIss

Instituto de Matemáticas,

UNAM Ciudad Universitaria,

04510 Mexico D.F., Mexico

E-mail: christof@math.unam.mx
}

\author{
Bernard LECLERC \\ Laboratoire LMNO, \\ Université de Caen, \\ F-14032 Caen Cedex, France \\ E-mail: leclerc@math.unicaen.fr \\ Jan SCHRÖER \\ Department of Pure Mathematics, \\ University of Leeds, \\ Leeds LS2 9JT, UK \\ E-mail: jschroer@maths.leeds.ac.uk
}

